\title{
MIXING LAYERS AND COHERENT STRUCTURES IN VEGETATED AQUATIC FLOWS
}

by

Marco Ghisalberti

B.Sc. (Chemistry) / B.E. (Environmental Engineering, Hons)

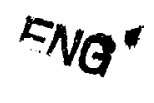

University of Western Australia, 1997

Submitted to the Department of Civil and Environmental Engineering

in partial fulfillment of the requirements for the degree of

Master of Science in Civil and Environmental Engineering

at the

MASSACHUSETTS INSTITUTE OF TECHNOLOGY

June 2000

(C) 2000 Massachusetts Institute of Technology. All rights reserved.

Author ..

Department of Civil and Environmental Engineering

May 12,2000

Certified by ..

Heidi M. Nepf

Associate Professor

I

Thesis Supervisor

Accepted by ..

MASSACHUSETTS INSTITUTE

OF TECHNOLOGY

MAY 302000
Daniele Veneziano

Chairman, Departmental Committee on Graduate Studies

\section{ENG}

\section{LIBRARIES}





\title{
MIXING LAYERS AND COHERENT STRUCTURES IN VEGETATED AQUATIC FLOWS
}

by

\author{
Marco Ghisalberti
}

\begin{abstract}
Submitted to the Department of Civil and Environmental Engineering on May 12, 2000 in partial fulfillment of the requirements for the degree of Master of Science in Civil and Environmental Engineering
\end{abstract}

\begin{abstract}
Submerged aquatic vegetation can dramatically alter the drag, turbulence and diffusivity characteristics of flow in aquatic systems. As a result, the diffusion and advection of contaminants and particulates are greatly influenced. However, modeling efforts generally treat submerged vegetation merely as a source of drag. This study explores the idea that flow through submerged aquatic vegetation resembles that of a mixing layer and can not be regarded simply as a perturbation of the bottom boundary layer.
\end{abstract}

A dynamically accurate experimental model of a submerged eelgrass canopy was created in a laboratory flume. The appropriateness of the mixing layer analogy was examined, with specific emphasis on the generation of large, coherent vortices above the vegetation. The vortices result in a strongly oscillatory flow and are responsible for the coherent waving phenomenon (monami) observed in terrestrial and aquatic vegetation. High frequency velocity records enabled the examination of the periodicity and turbulence characteristics of the vortices. These structures represent a very prominent feature of the flow and have the potential to dominate transport in vegetated areas.

Thesis Supervisor : Heidi M. Nepf

Title : Associate Professor of Civil and Environmental Engineering 



\section{ACKNOWLEDGEMENTS}

Thankyou to my advisor, Professor Heidi Nepf, for her enthusiasm, expertise and continued support throughout this work. Thanks must also go to fellow Nepf group members Chin Wu, Paul Fricker, Hrund Andradottir, Laura DePaoli and Brian White, whose comments, suggestions, advice and equipment tutorials were all invaluable.

This work was funded by a Gledden Postgraduate Studentship (from the University of Western Australia), a Schoetler Fellowship and an NSF Career Award EAR-9629259. Special thanks must go to the Gledden family, whose financial support helped make graduate study at M.I.T. a reality. 


\section{TABLE OF CONTENTS}

Abstract___ 3

Acknowledgements ___ 5

Table of contents _

List of figures____ 9

List of tables _ 10

Chapter 1. Introduction___ 13

1.1 Literature Review __ 15

1.1.1 Hydrodynamic effects of submerged vegetation __ 15

1.1.2 Coherent waving of vegetation__ 17

1.1.3 Mixing layer flow 18

1.1.4 The mixing layer nature of vegetated flows 22

1.1.4.1 Analysis of published data under the mixing layer framework __ 23

1.2 Objectives _ 26

Chapter 2. Experimental Methods __ 27

2.1 Experimental Configuration ___ 27

2.1.1 Inlet Conditions _ 28

2.1.2 Experimental model of aquatic vegetation__ 29

2.2 Instrumentation __ 30

2.2.1 Acoustic Doppler Velocimeter (ADV)_ 30

2.2.1.2 Use of the ADV in this study 32

2.2.2 Laser Doppler Velocimeter (LDV)__ 36

2.2.3 Comparison of the two methods of velocimetry ___ 41

2.3 Additional equipment

2.4 Data processing _ 43

2.5 Error analysis ___ 45

2.6 Dimensional analysis ___ 46

2.6.1 Balance of governing forces ___ 48

2.6.2 Further similarity criteria ___ 51

2.7 Plant properties ___ 53

2.8 Flow scenarios of the main experiments

2.8.1 Mean velocity profiles 56

2.8.2 Comparison of statistics obtained by the ADV and LDV__ 57

Chapter 3. Results and Discussion _ 59

3.1 Mathematical introduction ___ 59

3.2 Preliminary test __ 61

3.2.1 Presence of secondary circulation__ 65 
3.4 The appropriateness of the mixing layer analogy __ 69

3.4.1 Quantification of the mixing layer analogy 72

3.4.2 Important vertical length scales __ 74

3.4.3 Comparison of frequency spectra and monami frequencies _ 76

3.5 Vortex velocities __ 85

3.5.1 Effects of the rapid convective velocity of the vortices __ 91

3.5.2 Critical parameter for monami observation

3.5.3 Momentum transport by the coherent structures __ 95

3.5.3.1 Vortex size__ 99

3.5.4 Reverse shear in non-waving canopies__ 102

Chapter 4. Further Discussion 105

4.1 Differences between vegetated flow and laboratory mixing layers __ 105

4.2 Effects of depth limitation

4.3 Vortex kinematics _ 108

4.4 Frequencies of plant vibration

4.4.1 Effect of plant waving___ 109

4.5 Length scale prediction 110

4.6 Future work _ 111

Conclusion__ 113

References___ 115

Appendix A1 : MATLAB code for calculation of mean and turbulent parameters from ADV data

files 121

Appendix A2 : MATLAB code for calculation of mean and turbulent parameters from LDV data files 122

Appendix B : MATLAB code for calculation of smoothed spectra 123

Appendix C : MATLAB code for estimation of vortex velocity 125 


\section{LIST OF FIGURES}

Figure 1.1. Definition sketch of mixing layer variables 19

Figure 1.2. Kelvin-Helmholtz instabilities

(i) tilting tube

(ii) two gaseous streams 21

Figure 1.3. Vortex generated above a flexible vegetation layer

Figure 1.4. Comparison between observed frequencies and the expected frequency of the generated Kelvin-Helmholtz instability of a mixing layer 25

Figure 2.1. Experimental configuration in the recirculating laboratory flume 27

Figure 2.2. Schematic diagram of the ADV probe 31

Figure 2.3. Photograph of the method of data collection using the ADV 34

Figure 2.4. Fringe pattern within the sample volume of the LDV 38

Figure 2.5. Important geometric parameters of the model eelgrass blades 46

Figure 2.6. Photograph of the constructed model eelgrass meadow 55

Figure 3.1. Evolution of the vertical profile of mean longitudinal velocity as the flow encounters the model eelgrass meadow 62

Figure 3.2. Development of three fundamental mixing layer parameters as the flow progresses into the model canopy 64

Figure 3.3. (a) Schematic diagram of expected secondary circulation above model vegetation

(b) Evidence of mild secondary circulation generated in the flume 66

Figure 3.4. The conditions required for observation of the monami in the laboratory flume 68 Figure 3.5. Laterally averaged mean velocity profiles

(a) flow scenarios with the monami

(b) flow scenarios without the monami 70

Figure 3.6. Comparison between the observed velocity profiles and the traditional hyperbolic tangent profile of a mixing layer 71 Figure 3.7. Vertical profiles of $w_{r m s}$ 75

Figure 3.8. Pronounced peaks in the spectra of streamwise velocity in and above the model canopy 79 
Figure 3.9. Time series of a strongly oscillatory streamwise velocity record 80

Figure 3.10. Example of the double peak in the power spectrum of vertical velocity 81

Figure 3.11. Schematic diagram of the sequence of vertical velocities in the vortex street in vegetated flow

Figure 3.12. Schematic diagram of plant response to the passage of the street of elliptical vortices in the generated mixing layer

Figure 3.13. Experimental configuration for the measurement of the speed of progression of the coherent vortices

Figure 3.14. Examples of the cross-correlation between two lagged, simultaneous velocity records taken at different points along the canopy 89

Figure 3.15. Evidence of the linear relationship between the ratio of vortex velocity to mean mixing layer velocity with the non-dimensionalized mixing layer height Figure 3.16. Profiles of the correlation coefficient for flow scenarios with the monami__ 96 Figure 3.17. Profiles of the correlation coefficient for flow scenarios without the monami_ 97 Figure 3.18. Correlation coefficient profiles through the mixing layer for all flow scenarios with the monami 100

Figure 3.19. Correlation coefficient profiles through the mixing layer for all flow scenarios without the monami 101

Figure 3.20. Evidence of reverse shear in the non-waving canopies of this study 103

Figure 4.1. Evidence of the linear relationship between the observed momentum thicknesses in this study and the depth of the surface layer above the canopy 111

\section{LIST OF TABLES}

Table 2.1. Important features of the nominal velocity ranges for the $A D V$ 35

Table 2.2. LDV settings employed in this study 39

Table 2.3. Important parameters of real eelgrass meadows 47

Table 2.4. Important physical parameters of the model eelgrass meadow 54 Table 2.5. Flow and plant height parameters for the nine experimental runs comprising this study 
Table 3.1. Important mixing layer parameters of the mean velocity profile for all flow scenarios

Table 3.2. Contrast between the vertical length scales of the mixing layer flow and plant canopy

Table 3.3. Comparison of observed monami frequencies, predicted mixing layer instability frequency and the frequency of peaks in velocity spectra 83

Table 3.4. Estimation of vortex velocity based on crosscorrelation of two velocity records at different longitudinal locations 90 


\section{Chapter 1. Introduction}

Aquatic vegetation can dramatically alter the hydrodynamics of an aquatic system and strongly affect the advection and diffusion of both dissolved and particulate species. Consequently, aquatic vegetation can have a dramatic influence on water quality and has become an important parameter in constructed wetlands and coastal modeling; as sediments have such a strong influence on wetland physics, chemistry and biology, the understanding of near-bottom flow regimes is of utmost importance. The extent of research into the hydrodynamic effects of submerged aquatic vegetation, however, does not reflect the importance of the topic.

The majority of research into vegetated flows has examined the effect of terrestrial vegetation on atmospheric flows and the transport of momentum and scalar quantities into the canopies. While this situation is analogous to that of submerged aquatic vegetation, it must be noted that atmospheric flows are essentially unbounded vertically. The same is obviously untrue for aquatic flows that are bounded by the free surface. Of particular interest in this area of research, therefore, is the transition from fully bounded flow (emergent vegetation) to essentially unbounded flow (thoroughly submerged vegetation). Due to tidal effects, coastal vegetation can experience wide variability in its degree of submergence every day.

Seagrasses are a very prominent form of submerged aquatic vegetation, binding millions of acres of shallow sediments in the coastal waters of the United States (Fonseca, 1998). Eelgrass (Zostera marina), a species of seagrass, is abundant along the East coast of the United States and forms important ecological habitats in coastal regions (den Hartog, 1970). Zostera marina was thus chosen as a representative form of submerged aquatic vegetation for examination in this study. 
The impact of depth and current variation on vegetated flow forms the basis of this study. Specifically, however, this research was prompted by the observation of strong, coherent waving in seagrass meadows, both in the field and in the laboratory. This large-amplitude periodic waving is present under unidirectional flow and progresses smoothly along the canopy. The significance of such a prominent plant motion was expected to be great and the mechanism behind the plant waving represented an area of research of great importance and interest.

The underlying theme of this study is the analogy between flows through submerged vegetation and mixing layer flows developed by Raupach et al. (1996) for terrestrial canopies. Using this framework, the prominent features of flow through aquatic vegetation will be examined using an experimental eelgrass canopy constructed in a laboratory flume. The results of this study are applicable to all forms of flow through submerged vegetation, from aquatic vegetation of different flexibility and geometry to terrestrial vegetation. The limitations of the experimental model and its application to other vegetated flows cannot be stressed too heavily, however. This study focuses solely upon the interaction of the vegetation with a unidirectional flow, ignoring (for the time being) the potentially important role of surface waves. This, however, represents a critical area of future research.

This thesis is separated into four chapters. Chapter 1 gives insight into the motivation behind this research and a brief literature review on related topics that have been directly useful in this work. Chapter 2 discusses the experimental methods used in this study, from the experimental configuration in the laboratory to the benefits of the instrumentation that was employed. Presented in Chapter 3 are the complete set of results for this experimental study, along with explanatory discussion. Chapter 4 provides additional discussion on some particularly interesting discoveries and attempts to tie various aspects of this study together. 


\subsection{Literature Review}

\subsubsection{Hydrodynamic effects of submerged vegetation}

Aquatic vegetation can greatly affect the fate and transport of sediment, nutrients, contaminants, dissolved oxygen and fauna by altering the hydrodynamic conditions (Fonseca and Kenworthy, 1987; Nepf, 1999). Currents bend the seagrass into a streamlined shape, resulting in re-direction of the flow over the canopy (Fonseca et al., 1982; Gambi et al, 1990). The drag provided by the vegetation creates low velocity regions within the vegetation and promotes sediment deposition (Fonseca and Kenworthy, 1987; Gambi et al., 1990). Seagrass cover also decreases physical stress on the sediment-water interface, thus reducing erosion and stabilizing the benthos (Murota et al., 1984; Gambi et al., 1990; Fonseca, 1998). In addition, under most conditions, the leafy canopy inhibits resuspension of fine particles and traps suspended material, cleansing the water column of both sediment and nutrients (Fonseca, 1998). The local hydrodynamics can, in turn, have a strong influence on seagrass production, growth rate and photosynthesis (Fonseca and Kenworthy, 1987). The population dynamics of macrophytes are themselves important since these plants comprise about two-thirds of oceanic biomass and may serve as a considerable global carbon sink (Ackerman and Okubo, 1993).

The vertical inhomogeneity of the exerted drag leads to the development of strong velocity shear at the top of the vegetation canopy (Gambi et al., 1990; Grizzle et al., 1996; Vivoni, 1998; Wallace et al., 1998). Strong velocity shear therefore exists at the canopy-water interface, resulting in greatly increased turbulent intensities in this region, relative to unobstructed flow (Gambi et al., 1990; Vivoni, 1998; Wallace et al., 1998). Vertical turbulent transport of momentum into seagrass canopies has tremendous physical implications as it governs oxygen exchange, seed dispersal, sediment deposition and scalar fluxes within

seagrass beds (Wallace et al., 1998). The presence of large-scale turbulence above seagrass 
canopies has been noted by Ikeda and Kanazawa (1996) and Wallace et al. (1998); similarly, organized, coherent structures above terrestrial canopies have been identified by Raupach et al. (1996). Murota et al. (1984) concluded that turbulent motions produced immediately above the canopy dominate the structure of turbulence in vegetated open-channel flows. Consequently, these coherent structures are expected to dominate scalar and momentum transport in flows through aquatic canopies.

Studies of atmospheric flows through terrestrial vegetation are prevalent (e.g. Finnigan and Mulhearn, 1978; Finnigan, 1979a; Finnigan 1979b; Raupach et al., 1996) and provide a fairly extensive knowledge base for vegetated fluid flows. Raupach et al. (1996) confirmed that canopy turbulence is far from random with coherent eddies of canopy scale contributing greatly to the turbulent motions. Gao et al. (1989) found that coherent structures above a forest, consisting of a weak ejection from the canopy followed by a strong sweep into the canopy, contributed up to $80 \%$ of the momentum and heat fluxes at the canopy height. Similarly, Finnigan (1979b) revealed the dominant role of strong sweep events in momentum transfer into a terrestrial canopy. Furthermore, Raupach and Shaw (1982), showed that wake generation does not constitute a significant fraction of the turbulent kinetic energy (TKE) inside an atmospheric canopy. Wake turbulence is dissipated rapidly due to its small length scale, and the TKE inside the canopy is dominated by larger scale turbulence from above.

However, in atmospheric canopies the fluid layer is essentially unbounded vertically, meaning that the vegetated flow is superimposed upon an atmospheric boundary layer of a much larger scale. Conversely, in aquatic flows the ratio of water depth to plant height $(\gamma)$ is generally sufficiently small such that the vegetation affects flow throughout the entire water column. Vivoni (1998) examined the transition of thoroughly bounded flow (emergent vegetation) to essentially unbounded flow (high values of $\gamma$ ), as may be encountered in a typical tidal cycle. He found that there can be considerable depth-limitation on the turbulent structure 
of the flow through the aquatic canopy. The penetration of Reynolds stress into the canopy increases little above $\gamma \sim 2$, indicating this value as important in the depth-limitation transition.

Traditional treatments of the effect of aquatic vegetation on flow deal primarily with the

drag exerted by the plants and the discharge capacity of vegetated channels (e.g. Kouwen and Unny, 1973; Kouwen and Li, 1980). Such analyses consider the vegetation as perturbation of the bottom boundary and set about estimation of boundary layer properties such as friction velocity and Manning's $n$. Similarly, models of wetland hydrodynamics limit the effect of vegetation on the flow to the drag exerted by the canopy (e.g. DePaoli, 1999). While such an analysis has undoubted applications, it does not describe the effect of submerged vegetation on the turbulence structure and vertical momentum transport in the flow.

\subsubsection{Coherent waving of vegetation}

Seagrasses exhibit a synchronous, large-amplitude waving in response to water currents (e.g. Fonseca and Kenworthy, 1987; Grizzle et al., 1996). Accordingly, Ackerman and Okubo (1993) named this phenomenon 'monami'; the prefix mo- is Japanese for 'aquatic plant', the suffix -nami meaning 'wave'. This is the aquatic equivalent of the honami (coined by Inoue, 1955), the coherent waving of terrestrial vegetation, such as crop fields. Finnigan and Mulhearn (1978) found strong oscillations of velocity and Reynolds stress occurring at the top of a terrestrial canopy at the same frequency as plant waving. Finnigan (1979a) estimated, from video footage, that the velocity of the progression of the honami was approximately 1.8 times the mean velocity at the top of the canopy. The author proposed that as strong sweep events progress along the canopy, they depress a series of plants in their downwind passage. The plants then spring back and vibrate at their natural frequency.

Ackerman and Okubo (1993) identified the coherent waving of an eelgrass canopy at Woods Hole, MA. Grizzle et al. (1996) identified the same coherent waving in eelgrass beds at 
the mouth of the Jordan River, ME (at frequencies between 0.12 and $0.19 \mathrm{~Hz}$ ) but, significantly, only under maximum velocities within a tidal cycle. The reader is referred to Ackerman and Okubo (1993) for a photograph of coherent waving in a seagrass meadow in Craig Key, FL. Several researchers (Murota et al., 1984; Ackerman and Okubo, 1993; Grizzle et al., 1996; Wallace et al., 1998) have also identified significant, characteristic peaks in the spectrum of streamwise velocity through aquatic vegetation, ranging between 0.12 and $0.6 \mathrm{~Hz}$. Wallace et al. (1998) demonstrated a maximum energy of such oscillations at a height immediately above the canopy. Ackerman and Okubo (1993) postulated that the periodic velocity fluctuations were caused by the waving of the plants. However, this study demonstrates the presence of the converse causal relationship, specifically that the coherent waving of the plants is a response to strong oscillations in streamwise velocity.

Given the prominence and coherence of plant waving, the effect of the monami on the turbulence structure in vegetated flow was expected to be significant. Several researchers (e.g. Grizzle et al., 1996) have alluded to such a relationship, invariably, however, in the absence of conclusive evidence. Vivoni (1998) found the monami phenomenon had no effect on the turbulence structure in a model seagrass meadow, the monami considered a response to the system forcing rather than a dynamically significant interaction between the flow and the flexible plants. However, the waving of the stiff model plants of Vivoni (1998) was of a much lower amplitude than is expected in real eelgrass meadows (Grizzle et al., 1996). Therefore, the effect of pronounced, coherent waving on the turbulence structure in a seagrass meadow remains undetermined.

\subsubsection{Mixing layer flow}

The mixing layer consists of two regions of constant velocity, separated by a confined region of shear with an inflection point in the mean velocity profile. A schematic diagram of a mixing layer is shown in Figure 1.1. 


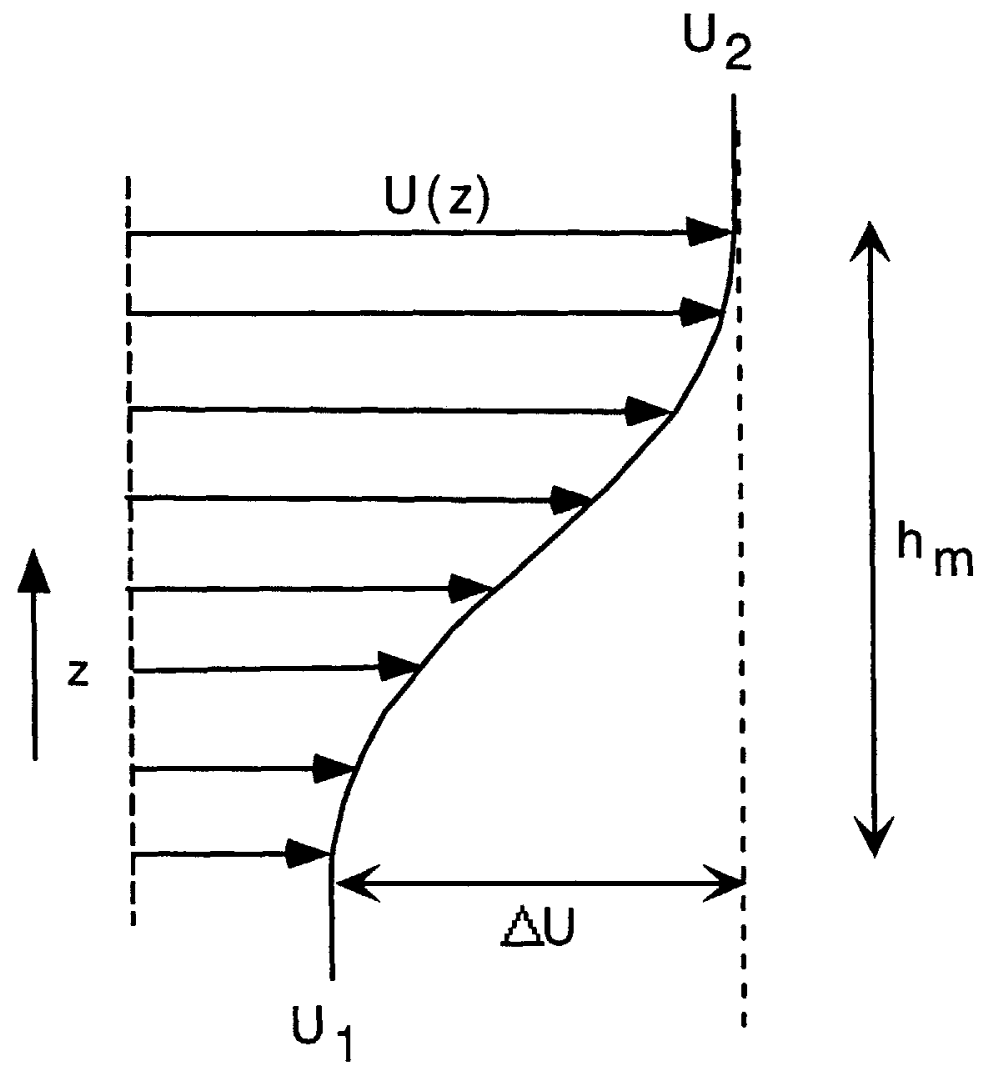

Figure 1.1. Definition sketch of mixing layer variables.

$U_{1}$ and $U_{2}$ are the mean low- and high-stream velocities respectively, $\Delta U$ is the difference between the two and $h_{m}$ is the height of the mixing layer, with nominal end-points defined by $\frac{U-U_{1}}{\Delta U}=0.01$ and $\frac{U_{2}-U}{\Delta U}=0.01$. In this study, $\bar{U}$ is defined as the arithmetic mean of $U_{I}$ and $U_{2}$ and $\bar{z}$ as the height above the bottom at which $U=\bar{U}$. Note that the velocity profile of a typical mixing layer is approximately that of a hyperbolic tangent (e.g. Ho and Huerre, 1984). It is thus symmetrical, with an inflection point in the profile at $\bar{z}$.

Rayleigh proved that a necessary (but not sufficient) criterion for instability of an inviscid parallel flow is that the basic velocity profile has a point of inflection (Kundu, 1990). 
Fjortoft subsequently discovered a more stringent, necessary condition for inviscid instability, namely that the magnitude of the vorticity must have a maximum within the region of the flow, not at any boundary (Kundu, 1990). Note that in free shear flows, viscous effects are not significantly stabilizing and the inviscid analysis describes the stability characteristics of the viscous flow well (Kundu, 1990). Both criteria are satisfied by typical mixing layer velocity profiles (e.g. hyperbolic tangent, error function). Mixing layers are commonly found in environmental flows; strongly stratified flows and the merging of two coflowing streams often have mixing layer profiles.

Mixing layers are subject to Kelvin-Helmholtz instability at every stage in their development (Holmes et al., 1996). This wave instability grows until it billows to form two-dimensional rollers (Ho and Huerre, 1984; Holmes et al., 1996). This has been visually demonstrated by a host of experimentalists (e.g. Brown and Roshko, 1974). Figure 1.1 shows the advanced nonlinear stage of the Kelvin-Helmholtz instability in a tilting tube experiment and in a shadowgraph of a gaseous mixing layer.

(i)

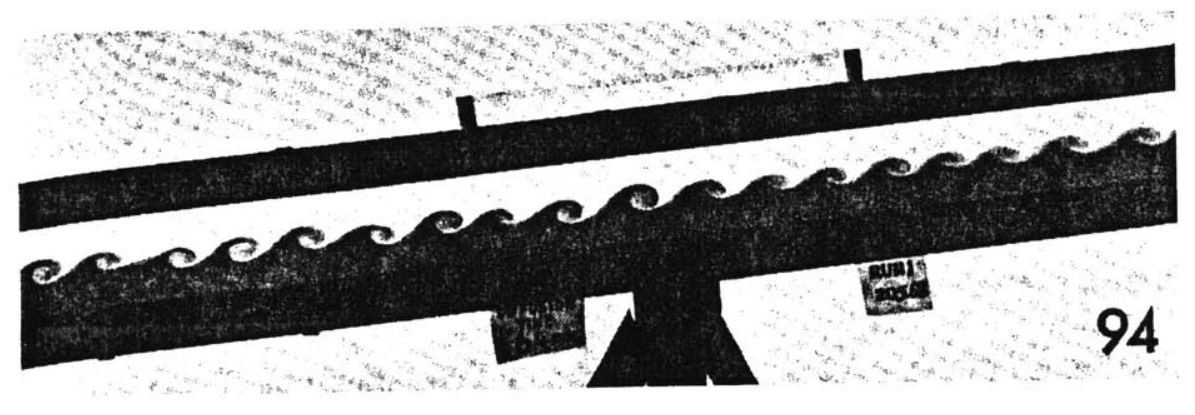


(ii)

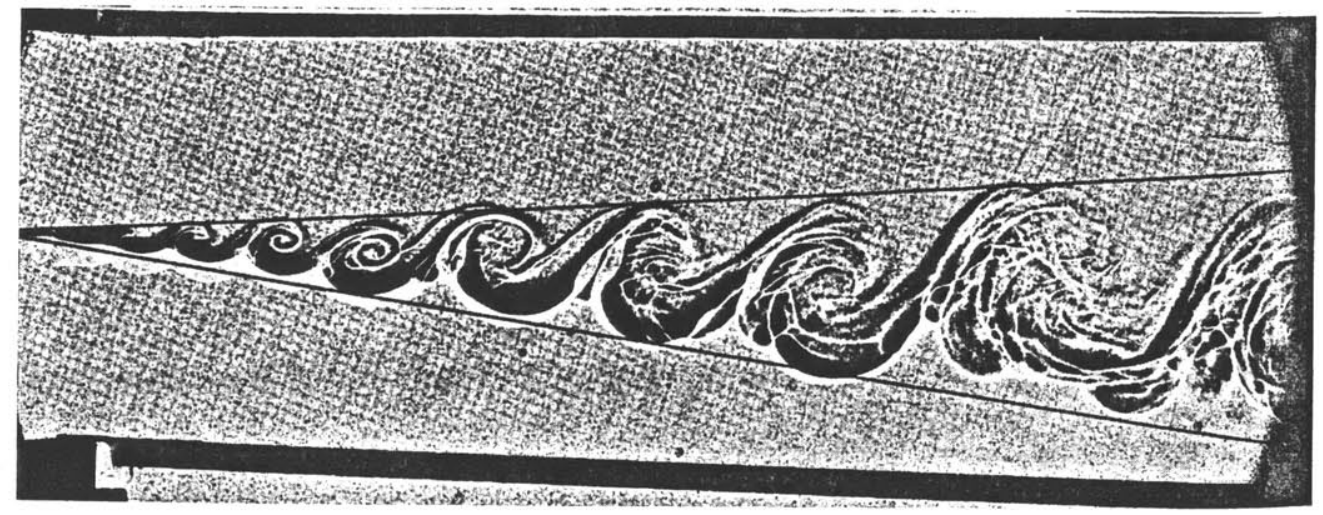

Figure 1.2. (i) Kelvin-Helmholtz instability generated in a tilting tube experiment. Mean flow in the lower layer is downslope and upslope in the upper layer (i.e. zero net flow) - from Thorpe (1971).

(ii) Shadowgraph of mixing layer between two gaseous streams (positive net flow from left to right) - from Brown and Roshko (1974).

After the generation of these vortices, neighboring rollers amalgamate under a stochastic pairing process (Winant and Browand, 1974; Brown and Roshko, 1974). This amalgamation, along with the entrainment of surrounding fluid by the vortices, is the mechanism behind the growth of mixing layers (Ho and Huerre, 1984). After two or three vortex pairings, a "mixing transition" occurs leading to fully-developed three-dimensional turbulence superimposed upon the coherent structures of the mixing layer (Brown and Roshko, 1974; Dimotakis and Brown, 1976). Beyond this transition, classical vortex pairings are no longer observed (Rogers and Moser, 1994), and 'tearing' (the destruction of a vortex by the engulfment of its vorticity by its neighboring vortices) is the mechanism of amalgamation. The thickness of laminar mixing layers increases with the square root of distance, while their turbulent counterparts grow linearly in space (Winant and Browand, 1974; Browand and Troutt, 1985); developed turbulent mixing layers evolve in a self-similar manner (Rogers and Moser, 1994). These vortices dominate the mass and momentum transfer through the mixing layer (Ho et al., 1991), although Browand and Troutt (1985) suggested that the vortex 
structure in turbulent mixing layers is comparatively weaker than the laminar instability, due to the turbulent diffusion of vorticity.

\subsubsection{The mixing layer nature of vegetated flows}

The publication that introduced the mixing layer analogy of flow through and above vegetation was that of Raupach et al. (1996). Until this point, vegetated flow was regarded as a perturbation of bottom boundary layer flow. The authors noted the similarity between mixing layer flow and flow through terrestrial canopies, by looking at several mean and turbulent velocity parameters. The inflection point in the mean velocity profile, the increased correlation between horizontal and vertical turbulent fluctuations and the strong skewness of the turbulent fluctuations in terrestrial canopies all pointed towards the mixing layer nature of flows through submerged vegetation. The momentum transfer of a mixing layer is dominated by sweeps on the low-velocity side of the flow, and by ejections on the high-velocity side; this corresponds with the turbulence structure observed in terrestrial canopies (Raupach et al., 1996) and submerged aquatic canopies (Vivoni, 1998).

Ikeda and Kanazawa (1996) built upon this analogy and examined the generation of organized vortices above flexible, aquatic vegetation. The three-dimensional vortices were found to be elliptical in cross-section; the flow field above their model vegetation is shown in Figure 1.3. The authors also proposed that as a vortex migrates downstream it generates the wavy motion of the vegetation. 


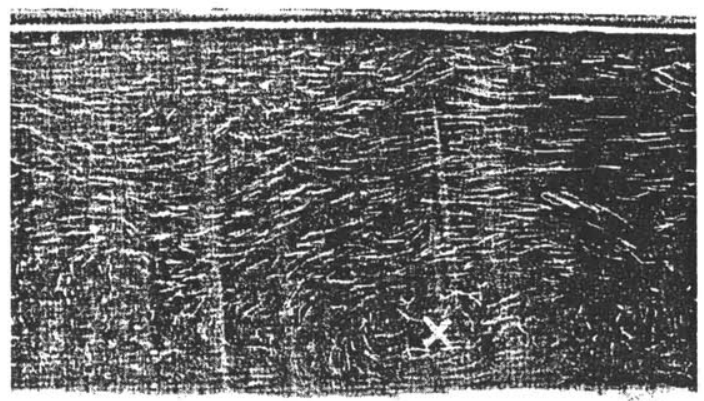

Free surface

Vegetation

layer

Figure 1.3. Vortex generated above a flexible vegetation layer (center of vortex indicated by cross). The flow visualization is accomplished with polystyrene beads (from Ikeda and Kanazawa (1996)).

\subsubsection{Analysis of published data under the mixing layer framework}

The momentum thickness of a mixing layer is defined as:

$$
\theta=\int_{-\infty}^{\infty}\left(\frac{1}{4}-\left(\frac{U-\bar{U}}{\Delta U}\right)^{2}\right) d z
$$

(Rogers and Moser, 1994)

and is an integral measure of the mixing layer thickness, although it certainly does not correspond to intuitive, visual estimates of that quantity. The linearized analysis of Ho and Huerre (1984) found that the frequency of the Kelvin-Helmholtz instability in a mixing layer, $f_{K H}$, was given by: 


$$
f_{K H}=0.032\left(\frac{\bar{U}}{\theta}\right)
$$

Equation (1.2) is conceptually reasonable; as the mean velocity increases, the advection speed of the vortices (and thus the observed frequency) will increase. Similarly, because the thickness of the mixing layer is linked to the size of the (momentum-transporting) vortices, a greater momentum thickness implies a greater vortex size. Therefore, fewer vortices will pass an observer per unit time if the momentum thickness is large.

Several publications on flow through aquatic vegetation provide a mean velocity profile from which the momentum thickness can be calculated. In several such publications, additional information has been provided regarding either:

(i) the frequency of the characteristic peak in the spectrum of streamwise velocity (Grizzle et al., 1996; Wallace et al., 1998),

(ii) the frequency of vortex generation above the canopy from flow visualization experiments (Ikeda and Kanazawa, 1996), or

(iii) the observed monami frequency (Vivoni, 1998). Note that the monami frequencies were obtained from this author's observation of video footage and differ from his published values.

Given this information, a comparison between the expected frequency of the mixing layer instability and the various observed frequencies can be made, as shown in Figure 1.4. The striking agreement demonstrated by this figure implies that not only are peaks in streamwise velocity spectra due to the Kelvin-Helmholtz instability of the shear layer, but also that this velocity oscillation causes the monami motion in aquatic vegetation. This appears to confirm the thoughts of Ikeda and Kanazawa (1996), who postulated that the downstream progression of the generated vortices was responsible for the wavy motion observed in their model canopy. 
The experiments of this study will seek to confirm this through analysis of spectra and observed monami frequencies. Note that the only field measurement of monami frequency shown in Figure 1.4 is that of Grizzle et al. and agrees with the field observations of Ackerman and Okubo (1993); namely, that a typical field monami frequency is of the order of $0.10-0.15 \mathrm{~Hz}$.

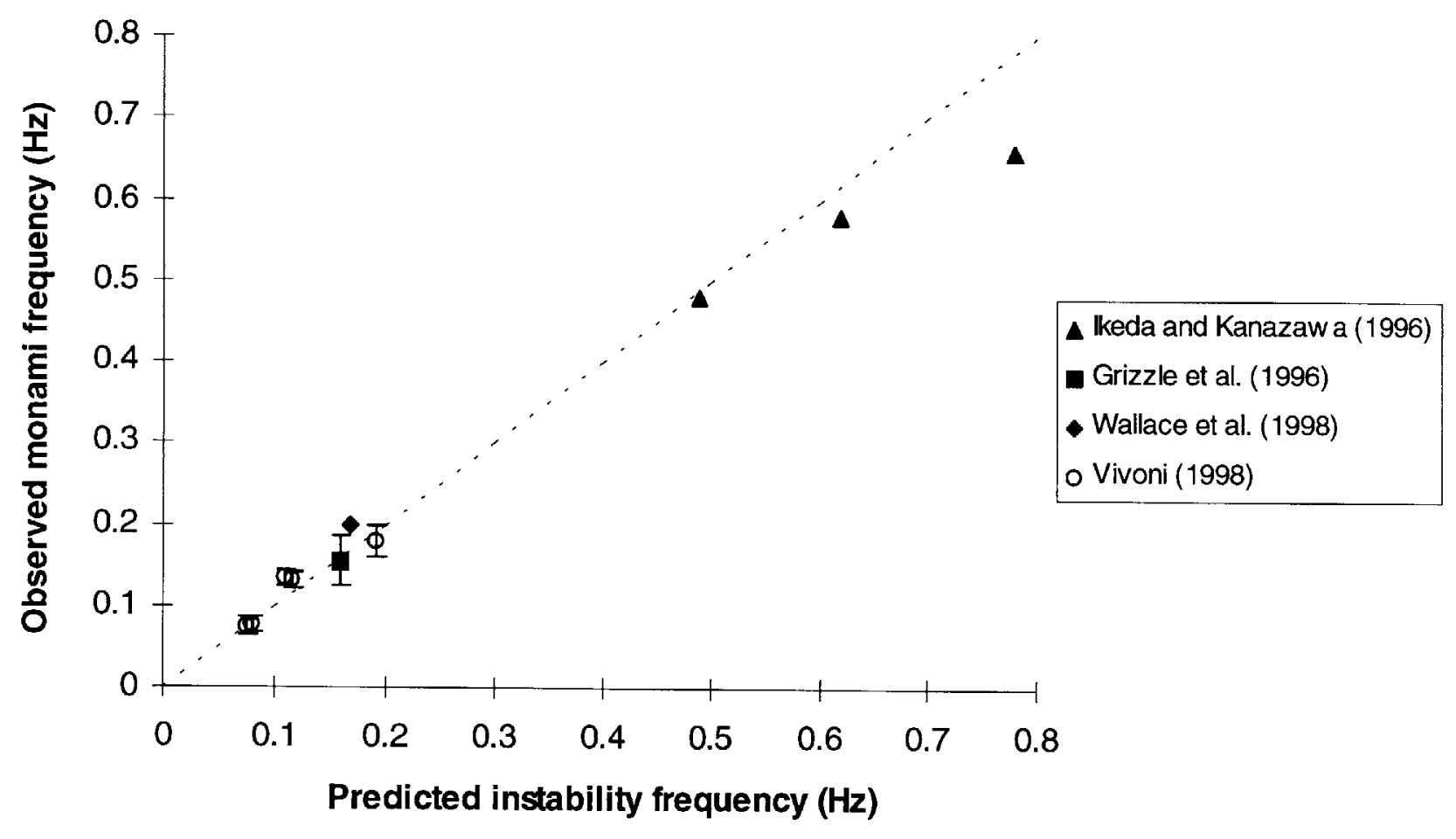

Figure 1.4. Comparison between observed frequencies and the expected frequency of the generated Kelvin-Helmholtz instability of a mixing layer. The dashed line represents perfect agreement. Vertical bars are indicative of uncertainty in the observed frequency. 


\subsection{Objectives}

The specific objectives of this study were to:

- Create a dynamically accurate model eelgrass canopy in the laboratory flume, for present and future use.

- Explain the observation of the monami phenomenon and determine the conditions required for its presence.

- Examine the appropriateness of the mixing layer analogy to flow through flexible, submerged aquatic vegetation.

- Determine the effect of plant waving on the turbulence structure, and specifically the vertical turbulent exchange, within and above the canopy. 


\section{Chapter 2. Experimental Methods}

\subsection{Experimental Configuration}

The experiments for this study were performed in a recirculating flume, in the Parsons Laboratory at M.I.T.. The glass-walled flume was $24 \mathrm{~m}$ long, $38 \mathrm{~cm}$ wide and $58 \mathrm{~cm}$ deep. The current in the flume was driven by a Weinman 3G-181 pump, whose flow rate could be adjusted between 10 and 240 gpm using a diaphragm valve. Flow rates were estimated using a Signet flow gauge, with an error of $\pm 3 \mathrm{gpm}$. The experimental configuration is shown in Figure 2.1 (note the vertical exaggeration). For the Cartesian coordinate system employed, $x=0$ was designated as the front of the model meadow, $y=0$ as the center of the flume and $z=0$ as the bottom of the model eelgrass bed.

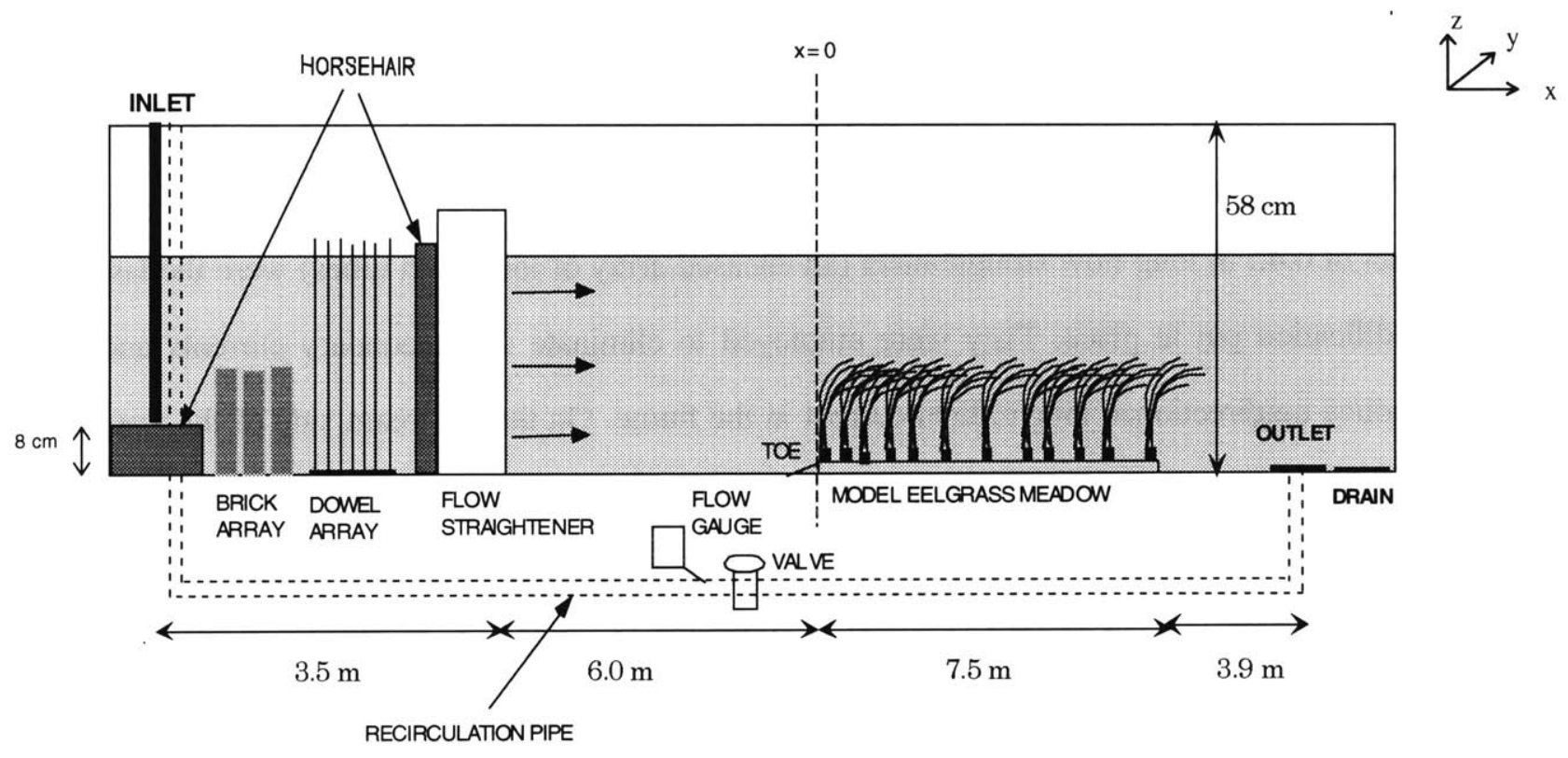

Figure 2.1. Experimental configuration in the recirculating laboratory flume. 


\subsubsection{Inlet Conditions}

Strong modifications were made to the flume near the inlet in an attempt to remove any inlet characteristics that may have persisted in the flow. To achieve this, the following modifications were made, as shown in Figure 2.1:

- Three sheets of rubberized coconut fiber ('horsehair') were stacked immediately under the inlet to rapidly dissipate turbulence generated at the inlet.

- Immediately downstream of the inlet, an array of 12 household bricks was put in place. This was done to provide a source of drag at the height of the inlet. The sheets of horsehair had the effect of diverting the discharge as a horizontal jet at $z=8 \mathrm{~cm}$ and the extra drag assisted in destroying the jet structure with the ultimate aim of achieving a smooth, monotonic velocity profile by the time the flow reached the model seagrass meadow.

- An $0.5 \mathrm{~m}$ long array of 100 surface-piercing dowels was placed downstream of the bricks. The function of the dowel array was two-fold: to break up any large scale turbulence signal imparted at the inlet and also to promote lateral and vertical uniformity in the flow conditions.

- A set of $0.45 \mathrm{~m}$ long flow straighteners (an encased array of long, thin tubes) were the last modification put in place. They were employed to eliminate any secondary currents and produce unidirectional, longitudinal current in the flume. On the upstream side of the flow straighteners, a vertical sheet of horsehair that spanned the entire flume cross-section was set in place. When the discharge was high, the sheets of horsehair underneath the inlet failed to prevent surface disturbance; the horsehair in front of the flow straighteners prevented the progression of surface waves further downstream. 


\subsubsection{Experimental model of aquatic vegetation}

The model eelgrass plants were attached to a series of six $1.2 \mathrm{~m}$ long Plexiglas boards; each board had two thousand $0.64 \mathrm{~cm}$ diameter holes drilled in random positions, with the restriction of being at least one diameter apart (Vivoni, 1998). The $38 \mathrm{~cm}$ wide boards were wedged in place with wooden dowels and affixed to the sidewalls of the flume using duct tape. The boards sat nicely on the flume bottom throughout the experiments. A small toe (1:8 slope) was placed at the front of the most upstream board to provide a smooth transition over the bottom displacement of $1.5 \mathrm{~cm}$.

Each model plant consisted of a stem region and six thin blades, based on the typical morphology of Massachusetts Bay eelgrass (Chandler et al., 1996). Short wooden dowels $(0.63 \mathrm{~cm}$ in diameter, $2.0 \mathrm{~cm}$ in height) were used to mimic the eelgrass stem, these dowels fitting snugly into the drilled holes. The model blades were cut from clear polyethylene film (AIN Plastics of New England) of thickness $(t) 0.10 \mathrm{~mm}$. The blades had a height $(h)$ of 13.0 $\mathrm{cm}$ and a width $(b)$ of $3.0 \mathrm{~mm}$, following the scaling arguments presented in Section 2.6.2. The lowest $3 \mathrm{~mm}$ of the blades were attached, with even spacing, to a small strip of duct tape, measuring $2.0 \mathrm{~cm}$ in length (i.e. exactly one circumference of the stem). A thin layer of waterproof glue was placed on the tape, the tape was wrapped around a dowel and secured with a small elastic band. This constituted the model eelgrass plant utilized for these experiments.

The model meadow consisted of 850 constructed plants, based on the scaling of densities of real eelgrass meadows, as discussed in Section 2.6.2. The plants were placed randomly into the holes in the Plexiglas boards, deliberate care being taken to avoid the creation of ordered arrays or staggered patterns. Real eelgrass meadows rarely have such structure, although localized clustering of eelgrass plants is prevalent. This clustering, however, is unlikely to affect the bulk, laterally-averaged velocity characteristics of the 
vegetated flow and plant placement in the model meadow was as random as possible. Once submerged, the wooden plant stems swelled, making their removal by the flow impossible.

\subsection{Instrumentation}

In this study, two forms of velocimetry were employed, namely acoustic doppler velocimetry and laser doppler velocimetry. Limitations of both techniques necessitated the concurrent use of the two methods during the experimental work.

\subsubsection{Acoustic Doppler Velocimeter (ADV)}

In fluid mechanics, studies of turbulence require current meters that can accurately measure all three velocity components with a sampling rate that is high enough to capture all turbulent fluctuations. The Acoustic Doppler Velocimeter (ADV), a versatile instrument capable of operating under both laboratory and field conditions, is widely used in studies of flow through aquatic vegetation for these reasons (e.g. Lopez and Garcia, 1997; Vivoni, 1998; Wallace et al., 1998).

The ADV probe consists of three receivers positioned in a horizontal circle around a 10 $\mathrm{MHz}$ transmitter. The receivers are slanted at $30^{\circ}$ from the axis of the transmitter, focusing on a cylindrical sample volume located approximately $5 \mathrm{~cm}$ below the probe. 


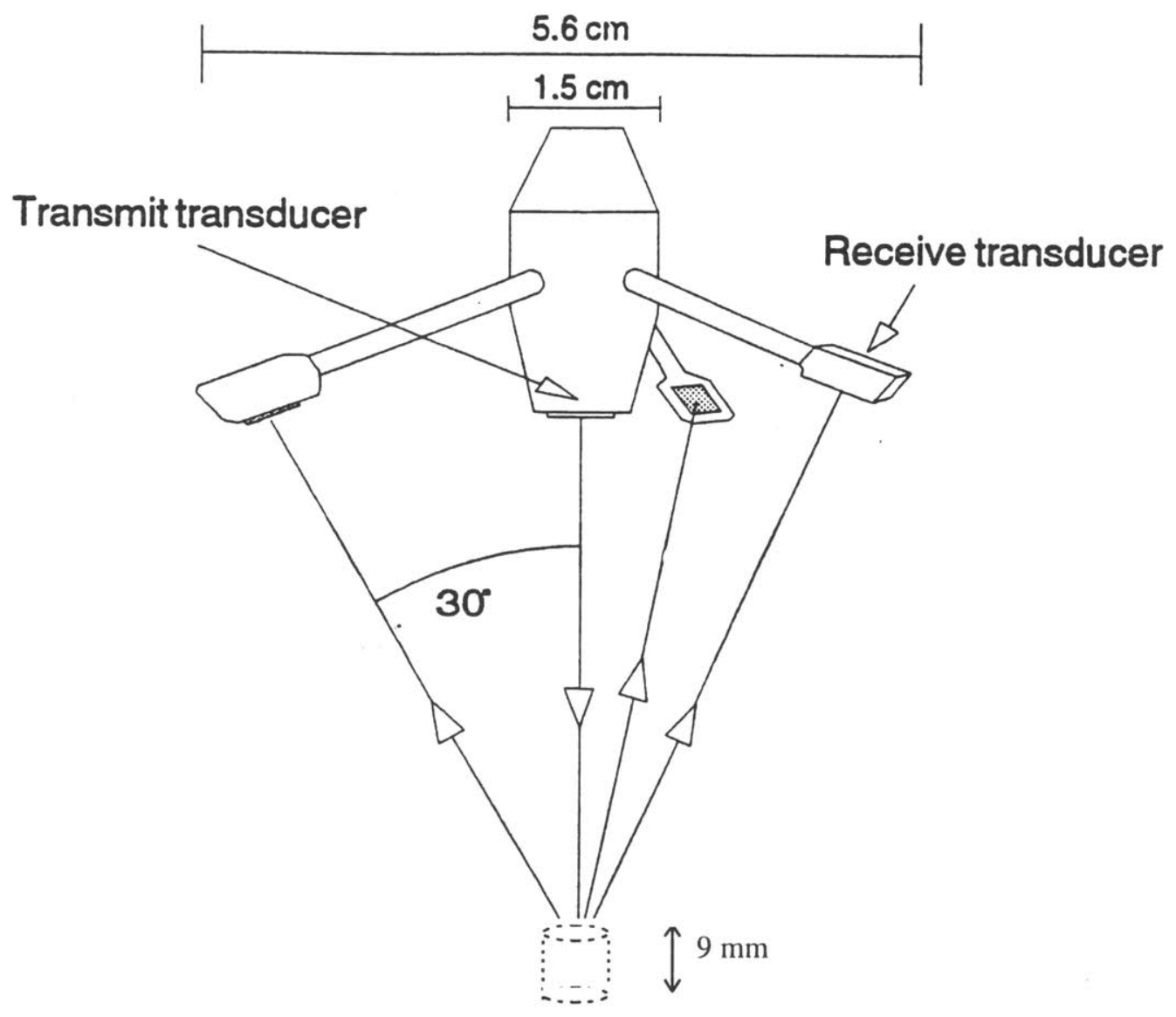

Figure 2.2. Schematic diagram of the ADV probe (from SonTek, 1996).

The probe is submerged in the flow and operates by transmitting short acoustic pulses; as the pulses propagate through the water, a fraction of the acoustic energy is backscattered by small particles, bubbles or suspended sediment (SonTek, 1995). When a moving particle scatters an acoustic signal, the frequency of the scattered signal is shifted; this is known as the Doppler shift. Whilst a particle or bubble remains in the sample volume, it is bombarded with a series of pulses. The three receivers detect the backscattering from the sample volume, with Doppler shifts proportional to particle velocities. The ADV probe is connected to a waterproof data processing module, which calculates the particle velocities from the measured Doppler shifts, using the Doppler relation: 


$$
V_{p}=\frac{c}{4 \pi f_{r}}\left(\frac{d \phi_{s}}{d t}\right)
$$

where $V_{p}$ is the velocity along the axis of the beam emanating from the sample volume towards each receiver $\left(\mathrm{ms}^{-1}\right)$, $c$ is the speed of sound in water $\left(\mathrm{ms}^{-1}\right)$, $f_{T}$ is the transmission frequency $\left(\mathrm{s}^{-1}\right)$, and $\phi_{s}$ is the phase of the backscattered signal (radians)

(Voulgaris and Trowbridge, 1997)

The speed of sound in water varies with water temperature and salinity; the former was measured with a Temprite alcohol thermometer and the latter was assumed to be zero. In this study, $c$ varied between $1469.4 \mathrm{~ms}^{-1}\left(T=16^{\circ} \mathrm{C}\right)$ and $1488.3 \mathrm{~ms}^{-1}\left(T=21{ }^{\circ} \mathrm{C}\right)$. The particle velocities along each beam axis are converted to the velocities along the Cartesian axes using the transformation matrix for the probe, which is based solely on probe geometry (SonTek, 1996).

The ADV system offers the advantage of being inherently drift-free, whilst requiring no routine calibration. The bottom edge of the ADV sampling volume can be placed within about $0.5 \mathrm{~mm}$ of a boundary (SonTek, 1995).

\subsubsection{Use of the ADV in this study}

In this study, a SonTek ADVField probe was used, the velocity resolution of which is $0.01 \mathrm{cms}^{-1}$. Data output from the ADV probe can be varied between 0.1 and $25 \mathrm{~Hz}$. As we are interested in various aspects of the turbulence structure of the flow, high frequency data is 
required to examine rapid velocity fluctuations. Consequently, data was obtained at $25 \mathrm{~Hz}$ during all experiments.

Before data collection, the ADV measures the distance (with an accuracy of about $1 \mathrm{~mm}$ ) to the flume bottom by measuring the time taken for strong reflection of an acoustic pulse (SonTek, 1995). The probe then corrects for all bottom reflection during data collection. For this reason, a small gap in the plant canopy directly below the probe was required at all times. This was achieved by the removal of up to 4 plants, creating a vacant area of up to 40 $\mathrm{cm}^{2}$. Ikeda and Kanazawa (1996) showed that such a removal of model plants has no significant effect on the flow conditions.

The underlying assumption of all forms of doppler velocimetry is that the velocity of the particle or bubble is equal to the velocity of the water. This assumption is generally valid, except in the presence of rapidly rising bubbles, which were absent in the flume during the experimental runs. For flume studies, the water must be 'seeded' with small particulates to achieve a sufficiently strong scattering signal. The relative strength of the signal is monitored by the signal-to-noise ratio (SNR), which is the strength of the backscattered signal minus the instrument noise level, in units of $\mathrm{dB}$ (SonTek, 1995). For this study, the SNR was maintained above 15 for all experiments, as recommended by SonTek (1996). This was achieved by periodic additions of a 1:5 w/v slurry consisting of fine titanium dioxide particles (provided by SonTek) in water. These particles are approximately neutrally buoyant in fresh water and are three orders of magnitude smaller than the length scales of the ADV sampling volume. This validates the underlying assumption of acoustic doppler velocimetry; namely, that the particles passing through the sample volume have an identical velocity to the water that carries them. 


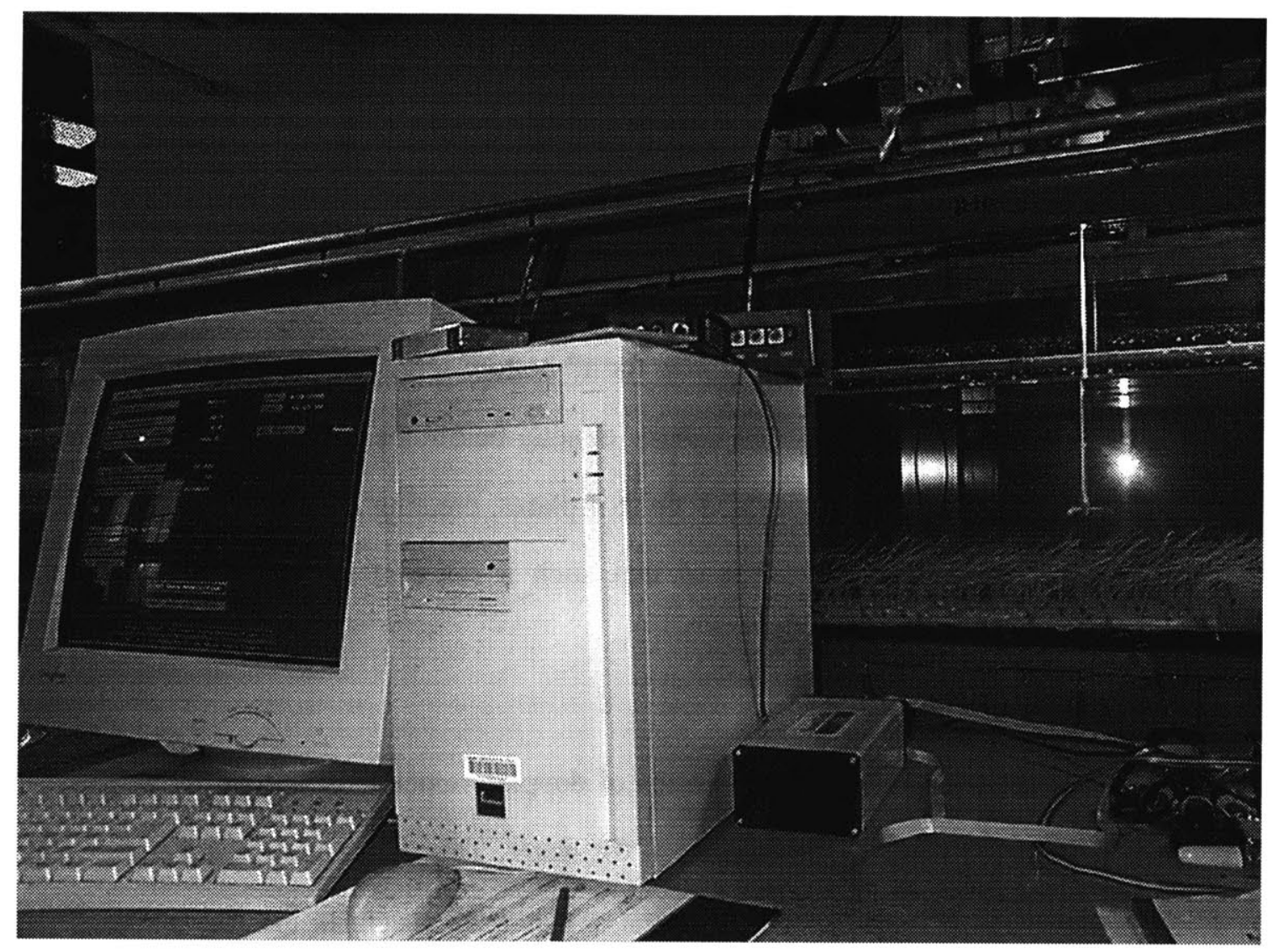

Figure 2.3. Photograph of the method of data collection using the ADV. The probe is linked to the processing module, which is in turn linked to a desktop PC for data retrieval.

The ADV probe was mounted on a trolley straddling the flume as shown in Figure 2.3. The probe was strapped (using tie-wraps) and affixed with duct tape to a vertical column, so as to prevent any twisting of the probe as it was moved vertically during the experiments. As shown in Figure 2.3, the data processing module was in turn linked to the serial port of a desktop computer for data retrieval. The interface program 'adfsx.exe', provided by SonTek, was used to adjust all settings and to visualize the time series of all three velocity components during the experiments.

The limitations of the ADV for resolving turbulent fluctuations is determined by the strength of the instrument-generated noise relative to the turbulent energy density. For vertical velocities, the instrument noise level is generally below typical turbulent energy densities over 
the entire frequency range. For horizontal velocities, it is common to see the beginning of a dominance of noise at a frequency of around $5-10 \mathrm{~Hz}$ for a sampling volume of this size (SonTek, date unknown). Fortunately, this study will concern itself primarily with velocity fluctuations of frequency $\sim 0.1 \mathrm{~Hz}$.

The SonTek ADV system allows the prescription of a velocity range setting. Because of the nature of the ADV Doppler processing, for each setting there exist maximum velocity levels above which the instrument cannot be relied upon to make accurate measurements (SonTek, 1995). However, the instrument noise increases with the nominated velocity range, as discussed in Voulgaris and Trowbridge (1997). Table 2.1 details the characteristics of the two lowest velocity ranges, ' $\pm 3 \mathrm{cms}^{-1}$ ' and ' $\pm 10 \mathrm{cms}^{-1}$ '.

\begin{tabular}{|ccccc|}
\hline $\begin{array}{c}\text { Nominal velocity } \\
\text { range }\left(\mathrm{cms}^{-1}\right)\end{array}$ & $\begin{array}{c}\text { Maximum horizontal } \\
\text { velocity }\left(\mathrm{cms}^{-1}\right)^{\mathrm{A}}\end{array}$ & $\begin{array}{c}\text { Maximum vertical } \\
\text { velocity }\left(\mathrm{cms}^{-1}\right)^{\mathrm{A}}\end{array}$ & $\begin{array}{c}\text { Instrument noise } \\
\left(\mathrm{cm}^{2} \mathrm{~s}^{-2}\right)^{\mathrm{B}}\end{array}$ & $\begin{array}{c}\text { Velocity error } \\
\left(\mathrm{mms}^{-1}\right)^{\mathrm{B}}\end{array}$ \\
\hline \pm 3 & \pm 30 & \pm 8 & $9.1 \times 10^{-3}$ & \pm 0.95 \\
\pm 10 & \pm 60 & \pm 15 & $9.5 \times 10^{-3}$ & \pm 0.97 \\
\hline
\end{tabular}

Table 2.1. Important features of the nominal velocity ranges for the ADV.

A (Sontek, 1995)

B (Voulgaris and Trowbridge, 1998)

Even allowing for strong turbulent fluctuations, the maximum velocities expected to be encountered in this experiment were well below those listed in Table 2.1. Consequently, the lowest velocity range (with the lowest velocity error and instrument noise) was chosen for all experimental runs in this study. 
Vivoni (1998) performed some tests on the same ADV probe as was used in this study and decided upon the optimal set of operating conditions for the probe. His findings are summarized below :

- In order to accurately estimate turbulent parameters such as the Reynolds stress and turbulence correlation coefficient, a critical parameter in this study, it is recommended that $\mathrm{ADV}$ records be 10 minutes long (i.e. 15000 points at a sampling frequency of $25 \mathrm{~Hz}$ ). For 10 minute records, the estimated mean square errors associated with mean velocity and Reynolds stress estimates are $0.6 \%$ and $1.2 \%$ respectively.

- As expected, the lowest velocity range setting resulted in improved probe performance. For velocity records taken upstream of the canopy, utilizing the $\pm 3 \mathrm{cms}^{-1}$ setting gave the lowest value of $u_{r m s}$ (i.e. the lowest standard deviation of the longitudinal velocity record). It was assumed that this was due to the lowest setting having the lowest amount of instrument noise.

\subsubsection{Laser Doppler Velocimeter (LDV)}

As the ADV control volume is located approximately $5 \mathrm{~cm}$ below the probe tip, velocities in the region immediately below the free surface could not be measured using this method. In this region, laser doppler velocimetry was required. For shallow water depths in the flume, the mixing layer can extend to within $\sim 1 \mathrm{~cm}$ of the free surface, making velocity measurements in this area critical.

Laser doppler velocimetry is, unlike its acoustic counterpart, an unintrusive method of measuring the instantaneous velocity of tracer particles suspended in the flow as they pass through a small sampling volume. Laser doppler velocimetry is based upon the photodetection of the backscattering of laser light by these particles while they remain within the sampling volume (Durst et al., 1981). The 60X Dantec Measurement Technology LDV employed in this study was two-dimensional, being able to measure longitudinal and vertical velocities only. 
The first component of the LDV system is the $300 \mathrm{~mW}$ blue-green argon ion laser (from Ion Laser Technology, Salt Lake City, UT). Within the Dantec optics system, the laser beam is split into two $488 \mathrm{~nm}$ blue beams (for the measurement of horizontal velocity) and two $514.5 \mathrm{~nm}$ green beams (for the measurement of vertical velocity). The beams are then focused into a fiber optic cable and travel along to the LDV probe; the blue beams exit the probe separated horizontally by $4.0 \mathrm{~cm}$, the green beams are separated vertically by the same distance. A lens within the probe focuses all four beams to a small sample volume (within the flow field) located approximately $20 \mathrm{~cm}$ from the lens. Due to the spatial Gaussian distribution of intensity of the laser beams, the sampling volume is elliptical; for this LDV system, it is 0.64 $\mathrm{mm}$ in length and $76 \mu \mathrm{m}$ in diameter (Dantec Measurement Technology, 1990a). The probe also detects backscattered light from the sample volume, whilst filtering out light scattering from outside the sample volume (Buchhave et al., 1979). Light backscatter is directed to the photomultipliers via the same fiber optic cable, and then to the burst correlation processor within the FVA (Dantec Measurement Technology, 1990a). At the intersection of two coherent laser beams, a fringe pattern is formed, as shown in Figure 2.4. Therefore, within the LDV sampling volume, the pairs of horizontally spaced blue beams and vertically spaced green beams each form a separate fringe pattern; the axis of the fringe pattern being perpendicular to the axis of the two beams. The spacing between the fringes $\left(d_{f}\right)$ is $2.18 \mu \mathrm{m}$ for the horizontal pattern formed by the blue beams and $2.07 \mu \mathrm{m}$ for the vertical pattern formed by the green beams (Vivoni, 1998). 


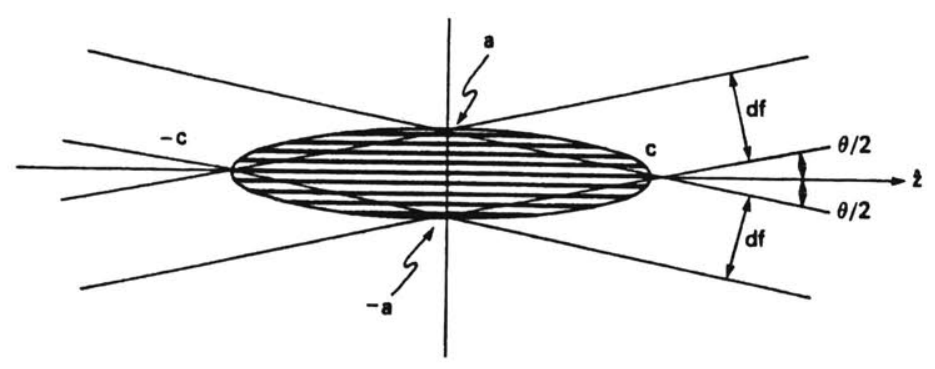

Figure 2.4. Fringe pattern within the sample volume of the $L D V$.

For each pairing, the frequency of one of the beams is shifted by $40 \mathrm{MHz}$ in a Bragg cell. This causes the fringe patterns to move within the sample volume, at a constant velocity much greater than that of any particle passing through the sample volume (Dantec Measurement Technology, 1990a). This allows the resolution of positive and negative particle velocities from the backscattering observed within the sample volume, since all particles will have velocities in the same direction relative to the rapidly moving fringe pattern.

As particles within the fluid pass through the fringe pattern within the sampling volume, the light scattered back towards the probe varies in intensity; obviously, the strength of the backscattered signal is stronger as the particle passes through a light band, and lower as it passes through a dark band. This cycle of backscattering intensity is known as the Doppler burst (Dantec Measurement Technology, 1990a). The particle velocity relative to the velocity of the fringe pattern $\left(v_{s}\right)$ in each direction is given by

$$
v_{s}=f_{D} \cdot d_{f}
$$

where $f_{D}$ is the frequency of the Doppler burst (Vivoni, 1998). Correction for the velocity of the fringe pattern enables calculation of the particle velocity as it passes through the sampling volume. 
The LDV settings shown in Table 2.2 were employed in this study. The high voltage level (HVL) determines the amplification of the scattering signal in the photomultipliers. As the HVL is increased, weaker Doppler bursts are identified, leading to an increased sampling frequency; however, the amount of background noise also increases. From the preliminary tests of Vivoni (1998), an HVL level of $1296 \mathrm{~V}$ would provide a decent sampling rate (expected to be $>60 \mathrm{~Hz}$ ) without incorporating excess background noise. The bandwidth controls the measurable velocity range; the next lowest setting $(0.12 \mathrm{MHz})$ corresponded to a velocity range of only $\pm 13 \mathrm{cms}^{-1}$, which may have been exceeded frequently during several runs. Consequently, the higher setting was used. The validation setting determines the signal-to-noise ratio above which a Doppler burst is considered to be due to a particle passing through the sampling volume. For the fringe spacing in the sampling volume of this LDV system, the minimum acceptable value is $-3 \mathrm{~dB}$ (Dantec Measurement Technology, 1990b); this value was consequently used throughout this study.

\begin{tabular}{|cc|}
\hline LDV setting & Value \\
\hline Bandwidth & $0.40 \mathrm{MHz}$ \\
Velocity range & $-41 \mathrm{cms}^{-1}$ to $+41 \mathrm{cms}^{-1}$ \\
High voltage level & $1296 \mathrm{~V}$ \\
Validation & $-3 \mathrm{~dB}$ \\
\hline
\end{tabular}

Table 2.2. LDV settings employed in this study.

The LDV probe was mounted on a tripod immediately adjacent to the sidewall of the flume at $x=6.5 \mathrm{~m}$. Again, this necessitated the removal of some of the model plants to prevent the blocking of the laser beams by the eelgrass blades. Up to 5 plants were removed in a thin sliver along the axis of the LDV probe such that all four beams arrived at the sampling volume with full intensity. The optics system was connected to a Dantec 58N40 Flow Velocity Analyzer, which uses a burst correlation processor to convert the optical signals into velocity 
data. The processor was in turn connected to a 58G130 Dantec FVA/PDA interface board, which allows the transfer of data to a PC with a Pentium processor. The interface board was installed in an IFA slot of the Dell PC used to collect and process the ADV data. The Flow 3.3 program, provided by Dantec Measurement Technology, was used to alter the sampling duration and to observe the histogram of velocity measurements during the sampling period.

Due to the method by which a velocity estimate is obtained, it was expected that slight seeding of the flume water would increase the sampling frequency without strongly diminishing the intensity of the laser beams at their confluence. When tested, this was observed to be true and the LDV measurements immediately followed the ADV measurements, without requiring replacement of the flume water.

The LDV offers the advantage of having a much smaller sampling volume thereby strongly reducing the amount of noise due to mean velocity shear within it. In addition, the mean sampling frequency of the LDV is significantly higher than that of the ADV. The recording of velocity data by the LDV is, however, very irregular, leading to data points unevenly separated in time.

The height of the LDV sampling volume above the flume bottom was measured with a ruler; the location of the sampling volume, at the confluence of the four laser beams, being immediately obvious. Heights were recorded with an error of $\pm 0.05 \mathrm{~mm}$. Due to the vertical separation of the green laser beams as they exit the probe, vertical velocities could not be measured within $1.5 \mathrm{~cm}$ from the free surface or bottom boundary. Longitudinal velocities could be measured to within $0.2 \mathrm{~cm}$ from the free surface or bottom boundary. For consistency, all velocity records taken with the LDV were 10 minutes in length. 


\subsubsection{Comparison of the two methods of velocimetry}

For this study, the ADV was the instrument of choice for the bulk of the velocity measurements, except in the $6-7 \mathrm{~cm}$ region immediately beneath the free surface. This decision was based on the work of Voulgaris and Trowbridge (1997) comparing the performance of an ADV and an LDV with semi-empirically predicted results for open channel flow. The authors showed that :

- The ADV sensor can measure both mean velocity and Reynolds stress to within $1 \%$ of the estimated 'true value'. While the LDV has a similar accuracy in the measurement of mean velocity, it tends to overestimate the Reynolds stress by a factor of two.

- Although some discrepancies were observed in the ADV measurement of longitudinal velocity variance, this was only for high velocity range settings, with a correspondingly high amount of noise. Vertical velocity variance was measured accurately using the ADV, especially at the lower settings.

- For boundary layer applications, the most significant noise term of the ADV is associated with the mean velocity shear within the sampling volume, which can become significant (compared to turbulent fluctuations) in regions of strong shear. However, even in regions very close to the boundary $(z \sim 1 \mathrm{~cm})$, the ADV provided sufficiently accurate estimates of the Reynolds stress, when compared to the 'true values'.

- Frequency spectra derived from ADV records agree well with theoretical spectra after correction for the spatial averaging within the ADV sample volume and the presence of a noise floor. For this study, the only quantitative feature of the frequency spectra that will be examined is the peak frequency. Although ADV spectra require some correction before agreement with theoretical prediction, the peak frequency is not compromised and will be accurately represented in all ADV spectra without any correction.

- Eddies smaller than $2.2 \mathrm{~cm}$ in the vertical and $1.5 \mathrm{~cm}$ in the horizontal are not fully resolved by the ADV sensor. However, given the canopy-scale coherent structures present in flow through submerged vegetation, eddies of this size are unlikely to significantly affect 
the flow in the mixing layer that develops. In the future, however, studies concerning stemand blade-generated wakes may require the use of the LDV.

For these reasons, the ADV was chosen as the main velocimeter for these experiments. For each run, measurements within $9 \mathrm{~cm}$ of the free surface were taken with the LDV. The top $7 \mathrm{~cm}$ were inaccessible using the ADV; two other records were taken at the same height as the uppermost ADV records to provide a means for comparison between ADV and LDV data.

\subsection{Additional equipment}

For each set of flow conditions, three 3-minute video recordings were taken of the eelgrass meadow (at $x=6.5 \mathrm{~m}$, under well-lit conditions) using a Hitachi Super 8 video camera. The first recording encapsulated up to $1 \mathrm{~m}$ of the meadow, in an attempt to obtain visual estimates of the progression speed of the monami. As a reference, transparent tape with $5 \mathrm{~cm}$ markings was placed along the sidewall of the flume. The second recording focused in upon $20 \mathrm{~cm}$ of the meadow in order to estimate the frequency of the monami, by observation of the progressive waving of a small longitudinal section of the canopy. The third recording was made with transparent tape, complete with $1 \mathrm{~cm}$ height markings, on the side wall of the flume. This was done so as to estimate the average height of the canopy; not only was there spatial variability in this parameter but also a strong temporal variation due to the presence of the monami. An approximate temporal and spatial average of the canopy height was estimated for all runs, by noting the height of 10 model plants during several different stages of the monami cycle. Given the intermittency and low frequency nature of the monami phenomenon, the recorded canopy height was never significantly less than the maximum canopy height during its waving cycle.

A Kodak DC50 digital camera was used to obtain the photographs seen in this thesis. The digital images were viewed and edited using the Kodak Picture Easy 2.0 software 
provided with the camera. All ADV and LDV data were extracted using a Dell $450 \mathrm{MHz}$ Pentium II PC, with Windows 98 platform.

\subsection{Data processing}

Data from the ADV is initially stored in binary files (*adv), which can then be converted into tabular ASCIl files (*.vel) using the 'getvel.exe' program provided by SonTek. These files were then ready for immediate import into MATLAB, where the bulk of the data analysis was performed (refer to Appendices A-C for the MATLAB codes that were generated). All data obtained using the aforementioned MATLAB codes was then exported into Microsoft Excel for presentation. Velocity data obtained by the LDV had to be extracted from the data file generated by the processor and was then exported into ASCII format, prior to analysis in MATLAB.

Before analysis of the velocity data, two separate corrections to the raw data were required. Firstly, correction for tilt of the ADV probe was employed. Even though the probe was aligned visually such that the longitudinal axes of the probe and flume coincided, with as little vertical tilt as possible, small angles of tilt were invariably introduced through the shifting of the probe. Consequently, small components of the longitudinal flow velocity were incorporated into the comparatively small lateral and vertical velocities, leading to significant errors in both. To counteract this, after each vertical profile of velocity records had been taken, a control velocity record was taken at a point $0.6 \mathrm{~m}$ in front of the canopy (Point A). Under the assumption that we have purely unidirectional flow before the canopy is encountered, a tilt-free probe should record zero mean lateral and vertical velocities at $\mathrm{A}$. The mean velocities recorded in front of the canopy were therefore used to estimate the tilt in the probe, assuming that the probe had not undergone any rotation. Letting $U_{\text {raw }}, V_{\text {raw }}$ and $W_{\text {raw }}$ denote the longitudinal, lateral and vertical velocities measured at A (with corresponding mean values 
$\bar{U}_{\text {raw }}, \bar{V}_{\text {raw }}$ and $\bar{W}_{\text {raw }}$ ), the horizontal and vertical tilt angles of the ADV probe ( $\alpha$ and $\beta$, respectively) were determined from simple trigonometric relationships:

$$
\begin{aligned}
& \alpha=\tan ^{-1}\left(\frac{\bar{V}_{\text {raw }}}{\bar{U}_{\text {raw }}}\right) \\
& \beta=\tan ^{-1}\left(\frac{\bar{W}_{\text {raw }}}{\bar{U}_{\text {raw }}}\right)
\end{aligned}
$$

Note that $\alpha$ and $\beta$ are defined positive in the direction of positive $y$ and $z$, as shown in Figure 2.1. Accordingly, the following corrections to the measured velocities in and above the canopy were made, as per Vivoni (1998):

$$
\begin{aligned}
& V=V_{\text {raw }} \cos \alpha-U_{\text {raw }} \sin \alpha \\
& W=W_{\text {raw }} \cos \beta-U_{\text {raw }} \sin \beta \\
& U^{*}=U_{\text {raw }} \cos \alpha+V_{\text {raw }} \sin \alpha \\
& U=U^{*} \cos \beta+W_{\text {raw }} \sin \beta
\end{aligned}
$$

Application of these corrections to the velocity records gathered at A leads obviously to corrected mean velocities of zero in both the lateral and vertical directions. Note that the tilt angles of the ADV probe remained fairly small throughout the experimental runs; the average values of $\alpha$ and $\beta$ were $1.5^{\circ}$ and $1.1^{\circ}$ respectively. Tilt correction was not required for the LDV data since visual alignment of the LDV probe was much simpler and significantly more accurate than for the ADV probe. The reflection of the laser beams from the back wall of the flume indicated quite clearly whether the probe was properly aligned. Before every individual velocity record was taken, the probe was carefully adjusted so that the blue and green beams were reflected at exactly the same height and longitudinal position (respectively) at which they were emitted. 
As a second correction, all LDV data had to be resampled, as this method records data at uneven intervals, as discussed in Section 2.2.2. Spectral analysis of data collected at a single frequency is much simpler and as shown by Vivoni (1998), this resampling has no significant effect on the evaluation of any turbulence parameters. Therefore, all velocity records were resampled at their mean sampling frequency $\left(f_{\text {samp }}\right)$, such that the total number of data points was conserved. That is, the $i$-th time data point of the of the resampled record $\left(t_{i}\right)$ is given by:

$$
t_{i}=\frac{i}{f_{\text {samp }}}
$$

Each new velocity data point was obtained by linear interpolation between the two velocity measurements taken at times surrounding $t_{i}$ in the raw velocity record.

\subsection{Error analysis}

Based on still water experiments, Vivoni (1998) showed that ADV estimates of mean velocity, Reynolds stress and vertical turbulent velocity would not be significantly affected by instrument noise. Horizontal turbulent velocity estimates showed evidence of contamination by noise but this was likely to become insignificant in turbulent flows. In addition, the spatial variability of these velocity statistics within and above a model seagrass canopy is much higher than instrument noise levels (Vivoni, 1998). Similarly, Zavistoski (1994) estimated the total error of an

LDV mean velocity measurement as $1.4 \mathrm{mms}^{-1}$, again expected to be negligible with respect to the spatial variability within and above the model canopy.

In the experiments of this study, all velocity statistics were laterally averaged; velocity records at three lateral positions in the flume were taken at each height under each flow scenario. This was done to capture the lateral variability in the flow, as the proximity to individual plants and their wakes varies considerably across a lateral traverse. Given the insignificance of instrument 
noise, any error quoted with velocity statistics represents solely the standard deviation of these three measurements.

\subsection{Dimensional analysis}

As with any experimental model, satisfaction of dynamic and geometric similarity with the prototype was critical. In this particular study, where the motion of the model plants had to be matched very closely to that of real eelgrass, dynamic similarity becomes very important. Note that in this section, the subscript $m$ refers to that parameter in the model eelgrass meadow, the subscript $p$ referring to that in the prototype (.e. in a real eelgrass meadow). The relevant geometric scales of the model eelgrass blades are described in Figure 2.5.

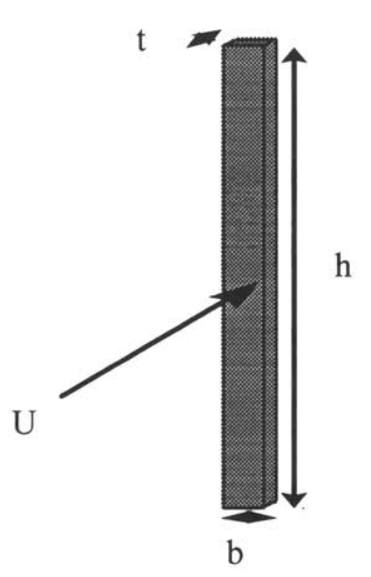

FRONT VIEW, UNDEFLECTED

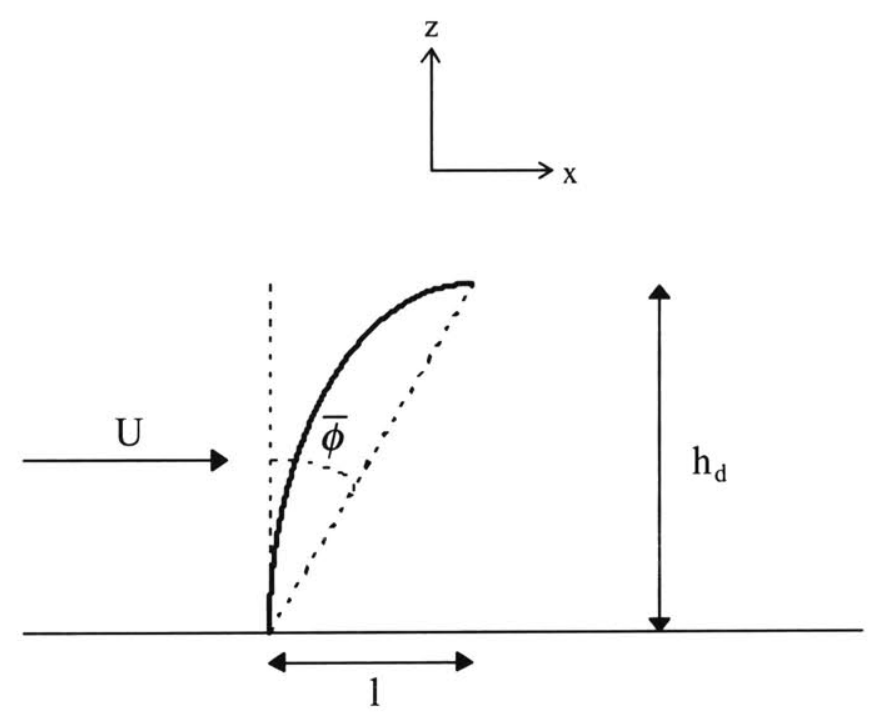

SIDE VIEW, DEFLECTED

Figure 2.5. Important geometric parameters of the model eelgrass blades. 
Achieving similarity in this study did not consist of simple numerical equation of several dimensionless parameters. Real eelgrass meadows exhibit wide ranges of many important parameters, as detailed in Table 2.3.

\begin{tabular}{|c|c|c|c|c|c|}
\hline Parameter & $\begin{array}{c}\text { Beaufort, } \\
\text { NC }^{\mathbf{A}}\end{array}$ & $\begin{array}{c}\text { Massachusetts } \\
\text { Bay }^{\mathbf{B}}\end{array}$ & $\begin{array}{c}\text { Chincoteague } \\
\text { Bay }^{\mathbf{C}}\end{array}$ & $\begin{array}{c}\text { Observed } \\
\text { range }^{\mathbf{D}}\end{array}$ & $\begin{array}{c}\text { Representative } \\
\left.\text { value }^{*}{ }^{*}\right)\end{array}$ \\
\hline $\begin{array}{c}\text { Areal density }(m), \\
\text { blades.m }\end{array}$ & - & 1800 & 3800 & $400-6000$ & 600 \\
\hline blade height $(h), \mathrm{cm}$ & 15 & 30 & 28 & $15-250$ & 30 \\
\hline $\begin{array}{c}\text { blade width }(w), \mathrm{cm} \\
\text { blade height } / \text { blade } \\
\text { width }(h / w)\end{array}$ & 0.30 & 0.36 & 0.26 & $0.25-0.5$ & 0.35 \\
\hline $\begin{array}{c}\text { Fraction of depth } \\
\text { occupied by } \\
\text { seagrass }(h / H)\end{array}$ & - & 83 & 108 & - & 80 \\
\hline
\end{tabular}

\section{Table 2.3. Important parameters of real eelgrass meadows.}

A (Fonseca, 1998)

B (Chandler et al., 1996)

C (Vivoni et al., 1997)

D taken from (Vivoni, 1998) and then modified using data from Grizzle et al. (1996) and Fonseca (1998)

* This value was not an arithmetic mean of the tabulated data, but simply a chosen value that represented typical field conditions, with particular attention paid to Massachusetts Bay conditions.

In addition, for many critical parameters (e.g. blade thickness, blade mass density) there have been precious few field measurements. Prof. Evamaria Koch of Horn Point Environmental 
Laboratory guessed at an average blade thickness, $t$, of $0.1-0.2 \mathrm{~mm}$ (pers. comm., 12 November 1999), while Fonseca (1998) estimated the blade mass density $\left(\rho_{s}\right)$ as $760 \mathrm{kgm}^{-3}$. Hence, real eelgrass blades are positively buoyant in both fresh and saline water, imposing a stringent criterion upon the material to be used.

\subsubsection{Balance of governing forces}

The motion of the plant blades is governed by a combination of a drag force $\left(F_{D}\right)$, a buoyancy force $\left(F_{B}\right)$ and a restoring force $\left(F_{R}\right)$ due to the deflection of the blades by the current. From the geometry of the blade,

$$
\begin{gathered}
F_{B}=\left(\rho_{s}-\rho_{w}\right) g \forall=\left(\rho_{s}-\rho_{w}\right) g h w t \\
F_{D}=\frac{1}{2} \rho_{w} A_{f} C_{D} U_{c}^{2} \approx \frac{1}{2} \rho_{w}(h w \cos \bar{\phi}) C_{D} U_{c}^{2}
\end{gathered}
$$

where $\rho_{w}$ is the density of water, $\forall$ is the volume of an individual blade, $A_{f}$ is the frontal area of the blade, $U_{c}$ is the mean in-canopy velocity and $C_{D}$ is the drag coefficient of the blade. The internal moment in a bent blade, $M_{I}$, is given by

$$
M_{I}=J \frac{\partial^{2} z}{\partial x^{2}}
$$

where $J$ represents the flexural rigidity of the blade and defines the resistance of the beam to bending. Flexural rigidity is defined simply as the product of the modulus of elasticity, $E$, and the moment of inertia, $I$, incorporating the stiffness of the material and the geometric resistance to bending: 


$$
J=E I
$$

The moment of inertia, $I$, for a beam of rectangular cross section (with width $w$ and thickness $t$ ) bending about its $y$-axis is given by

$$
I=\frac{1}{12} w t^{3}
$$

where all dimensions and directions are defined just as for an eelgrass blade in the flume.

From Equation 2.12 therefore,

$$
F_{R} \sim \frac{J}{h}\left(\frac{h-h_{d}}{l^{2}}\right)=\frac{J}{h l}\left(\frac{h}{l}-\frac{h_{d}}{l}\right) .
$$

Since

$$
l=\frac{h_{d}}{\tan \bar{\phi}} \approx h \cdot \sin \bar{\phi} \quad \text { for small deflections }
$$

Equation 2.15 becomes

$$
F_{R} \sim \frac{J}{h^{2} \sin \bar{\phi}}\left(\frac{1}{\sin \bar{\phi}}-\tan \bar{\phi}\right)=\frac{J}{h^{2}} \cdot f_{1}(\bar{\phi}),
$$

where

$$
f_{1}(\bar{\phi})=\left(\frac{1}{\sin ^{2} \bar{\phi}}-\frac{1}{\cos \bar{\phi}}\right)
$$

The most important dynamic dimensionless parameters in this study are the two independent ratios of the governing forces, namely: 


$$
\frac{F_{B}}{F_{R}}=\frac{\left(\rho_{s}-\rho_{w}\right) g \cdot h^{3} \cdot w \cdot t}{J \cdot f_{1}(\bar{\phi})}
$$

and

$$
\frac{F_{B}}{F_{D}}=\frac{2\left(\rho_{s}-\rho_{w}\right) g . t}{\rho_{w} \cos \bar{\phi} C_{D} U_{c}^{2}}
$$

As the angle of deflection of the eelgrass blades cannot be predetermined, it can simply be matched by adjusting the flow velocity.

Note that the drag coefficient of a plate aligned normal to the flow displays a very weak dependence on Reynolds' number (Gerhart et al., 1992); thus, the drag coefficient of eelgrass blades can be treated as a constant. Under the assumption, therefore, that $f_{1 m}(\bar{\phi}) \approx f_{1 p}(\bar{\phi})$ and $\cos \bar{\phi}_{m} \approx \cos \bar{\phi}_{p}$ is automatically prescribed, and ignoring the other parameters that are approximately equal in the field and in the model (i.e. $g, \rho_{w}$ and $\left.C_{D}\right)$, the dynamic parameters (now dimensional) become, respectively:

$$
\begin{gathered}
\lambda_{1}=\frac{\left(\rho_{s}-\rho_{w}\right) h^{3} \cdot w \cdot t}{J} \\
\lambda_{2}=\frac{\left(\rho_{s}-\rho_{w}\right) t}{U_{c}{ }^{2}}
\end{gathered}
$$

The material chosen for the construction of the eelgrass blades was polyethylene film, supplied by AIN Plastics of New England. This material was chosen because not only was it positively buoyant in the fresh water of the flume $\left(\rho_{s}=920 \mathrm{kgm}^{-3}\right)$ but it had a modulus of elasticity $\left(E=3.0 \times 10^{8} \mathrm{~Pa}\right)$ that would allow construction of suitably sized plants with a flexural rigidity comparable to that of real eelgrass plants. It was deemed that $\lambda_{l}$, more so than 
$\lambda_{2}$, was the determinant of plant motion; $\lambda_{2}$ depends upon the square of the canopy velocity which can be altered as required.

Given the uncertainty of several field parameters, a direct numerical matching of $\lambda_{1}$ was not sufficient to ensure similarity. Using our only set of field data that included $J_{p}$ $\left(=8 \times 10^{-8} \mathrm{kgm}^{-3} \mathrm{~s}^{-2}\right.$ from Fonseca (1998)), the preliminary estimate of $\lambda_{I}$ is 3 , but the value could conceivably range between 0.1 and 10 . To find the most realistic plant behavior, six test plants with $8 \mathrm{~cm}$ long blades and with a range of values of $\lambda_{l}(0.07$ to 1.1$)$ were constructed.

As a stringent examination of the model plants, the constructed plants were placed in the flume and subjected to a wave environment. The wave generator was used to generate sinusoidal waves of period $0.5 \mathrm{~Hz}$ and amplitude $2.0 \mathrm{~cm}$, both of which are appropriate scaled values for the laboratory flume. Video footage of actual eelgrass meadows in the open ocean, taken in April 1989, was obtained from Prof. Koch. This footage showed that eelgrass blades have a whip-like oscillation in a wave environment; the motion is certainly unlike that of a rigid beam oscillating about a pivot. Within the constructed plants, a range of behavior was observable, the stiffer plants (lower values of $\lambda_{I}$ ) lacking the whip-like motion of the real plants. The model plant with the most realistic behavior was that with $\lambda_{1}=0.66$.

\subsubsection{Further similarity criteria}

As shown by Vivoni (1998), dimensional analysis involving all system variables in the modeling of a seagrass canopy yields the following important dimensionless parameters (where $p_{i}$ represents the set of $\{h, w, t\}$ and $v$ is the kinematic viscosity of water):

$$
\frac{U H}{v}, h a, \frac{H}{h} \text { and } \frac{p_{i}}{H},
$$


given that matching the Froude number is both unimportant (Vivoni, 1998) and impossible if any form of Reynolds number similarity is desired.

Whilst matching of the blade height Reynolds number, $\operatorname{Re}_{h}(=U h / v)$, is desirable, it is difficult to do so with such a range of velocities experienced in the field and in these experiments. As a rough numerical comparison, the Reynolds number in the field (using the depth-averaged current) ranges from 0 to $5 \times 10^{6}$ (Vivoni, 1998). In this experiment, the range of $\operatorname{Re}_{h}$ was 1100 to 6000 , well within the observed range in the field. In addition, a simple matching of the flow regime in the open channel of the flume was also required. For all runs, the depth Reynolds number $(U H / V)$ was above 2000 , meaning the flow in the flume was always turbulent; one would always expect to see turbulent flow in the field.

The choice of the model blade height was constrained by the limited height of flume $\left(H_{f}=58 \mathrm{~cm}\right)$; a significant canopy height was desirable, as was a sufficiently deep surface layer above the canopy. Considering that an $H / h_{d}$ value of approximately 2 has been observed to be a point of transition in the turbulence structure of vegetated aquatic flow (Vivoni, 1998), an $H_{f} / h$ value of 3-4 was desired. Consequently, a blade height of $12.7 \mathrm{~cm}$ was chosen. Despite a representative $h / w$ ratio of 80 in the field (Table 2.3 ), the polyethylene film could only be consistently cut into $3 \mathrm{~mm}$ wide strips, giving $h /\left.w\right|_{m}=42$, which is not a huge deviation from the range observed in the field.

Scaling the areal plant density of the eelgrass meadow was based on the dimensionless parameter $h a$. The plant density parameter, $a$, describes the total frontal area per unit volume occupied by the plants:

$$
a=m b
$$


where $m$ is the number of blades per unit area. From Table 2.3, a typical value of $m$ in the field is 600 blades. $\mathrm{m}^{-2}$, a typical blade width $0.40 \mathrm{~cm}$, and a typical height $0.30 \mathrm{~m}$. Therefore, $(h a)_{p}=0.72$. Matching this parameter in the model, with a blade height of $12.7 \mathrm{~cm}$ and width of $0.30 \mathrm{~cm}$, meant $m_{m}$ was required to be 1890 blades. $\mathrm{m}^{-2}$, or 315 plants. $\mathrm{m}^{-2}$. With the canopy having an area of $2.7 \mathrm{~m}^{2}, 850$ model plants were required.

Maintaining a $\lambda_{I}$ value of 0.66 , the scaling of the $8 \mathrm{~cm}$ blades of the preliminary model plant to a height of $12.7 \mathrm{~cm}$ gave a required blade thickness of $0.10 \mathrm{~mm}$; polyethylene film of this thickness was, fortunately, commercially available. Thus, the dimensions of the model eelgrass meadow had been finalized.

\subsection{Plant properties}

A rigid beam clamped at one end has a fundamental, natural frequency of oscillation $\left(f_{\text {nat }}\right)$ given by

$$
f_{n a t}=\frac{(1.875)^{2}}{2 \pi}\left[\frac{E I}{m l_{b}^{4}}\right]^{1 / 2}
$$

(Niklas, 1992)

where $m$ is the mass per unit length of the beam $\left(\mathrm{kgm}^{-1}\right)$

and $l_{b}$ is the beam length $(\mathrm{m})$, which, in this case, corresponds to the blade height.

To determine $m$, a small section $(11.25 \mathrm{~cm} \times 30.40 \mathrm{~cm})$ of the polyethylene film was weighed. The mass of the section was $3.18 \mathrm{~g}$, giving a mass per unit area of $9.30 \times 10^{-3} \mathrm{gcm}^{-2}$. With the blades cut to a width of $0.30 \mathrm{~cm}$, their mass per unit length would therefore be $3.10 \times 10^{-2}$

$\mathrm{gcm}^{-1}\left(\equiv 3.10 \times 10^{-3} \mathrm{~kg} \cdot \mathrm{m}^{-1}\right)$. Therefore, given the value of $J$ in Table $2.4, f_{\text {nat }}=0.18 \mathrm{~Hz}$. 
It is expected, however, that the monami frequency is independent of the natural frequency of oscillation of the blades. The model plants of Vivoni (1998) had a natural frequency of $3.1 \mathrm{~Hz}$, yet video footage revealed a maximum monami frequency of just 0.18 $\mathrm{Hz}$. The video footage also showed a mild lateral vibration under strong currents; the frequency of this vibration was observed to be of the order of $2-3 \mathrm{~Hz}$, and may represent the natural frequency of the blades. No such lateral oscillation was observed in this study.

The important physical parameters of the constructed eelgrass meadow are summarized in Table 2.4 .

\begin{tabular}{|lcl}
\hline Blade properties & & \\
Mass density & $\rho_{s}$ & $920 \mathrm{kgm}^{-3}$ \\
Flexural rigidity & $J$ & $8.0 \times 10^{-8} \mathrm{kgm}^{-3} \mathrm{~s}^{-2}$ \\
Blade height & $h$ & $12.7 \mathrm{~cm}$ \\
Blade width & $w$ & $3.0 \mathrm{~mm}$ \\
Blade thickness & $t$ & $0.102 \mathrm{~mm}$ \\
Natural vibration frequency & $f_{n a t}$ & $0.18 \mathrm{~Hz}$
\end{tabular}

\section{Bulk canopy properties}

Undeflected plant height

$\begin{array}{ll}h_{\text {und }} & 13.5 \mathrm{~cm}\left(^{*}\right) \\ m_{m} & 315 \text { plants. }{ }^{-2}\end{array}$

Areal plant density

Table 2.4. Important physical parameters of the model eelgrass meadow.

* This value differs from that expected based on the height of the blades and dowels. Even in still water, blades were rarely fully erect, leading to a diminished undeflected height as seen here.

In summary, due to a dearth of field information, the dynamic dimensionless parameters (shown in (2.19) and (2.20)) were not used to directly obtain the model 
specifications. Rather, they were used to create a set of plants in the ballpark, the correct plant being chosen based on video footage of an actual eelgrass meadow. All observers thus far have commented on the realism of the plant behavior in the flume, generating confidence in the scaling methods used. A photograph of the model eelgrass meadow is shown in Figure 2.6. As more information on eelgrass meadows is published, the aforementioned dimensionless parameters may be used for simple numerical scaling.

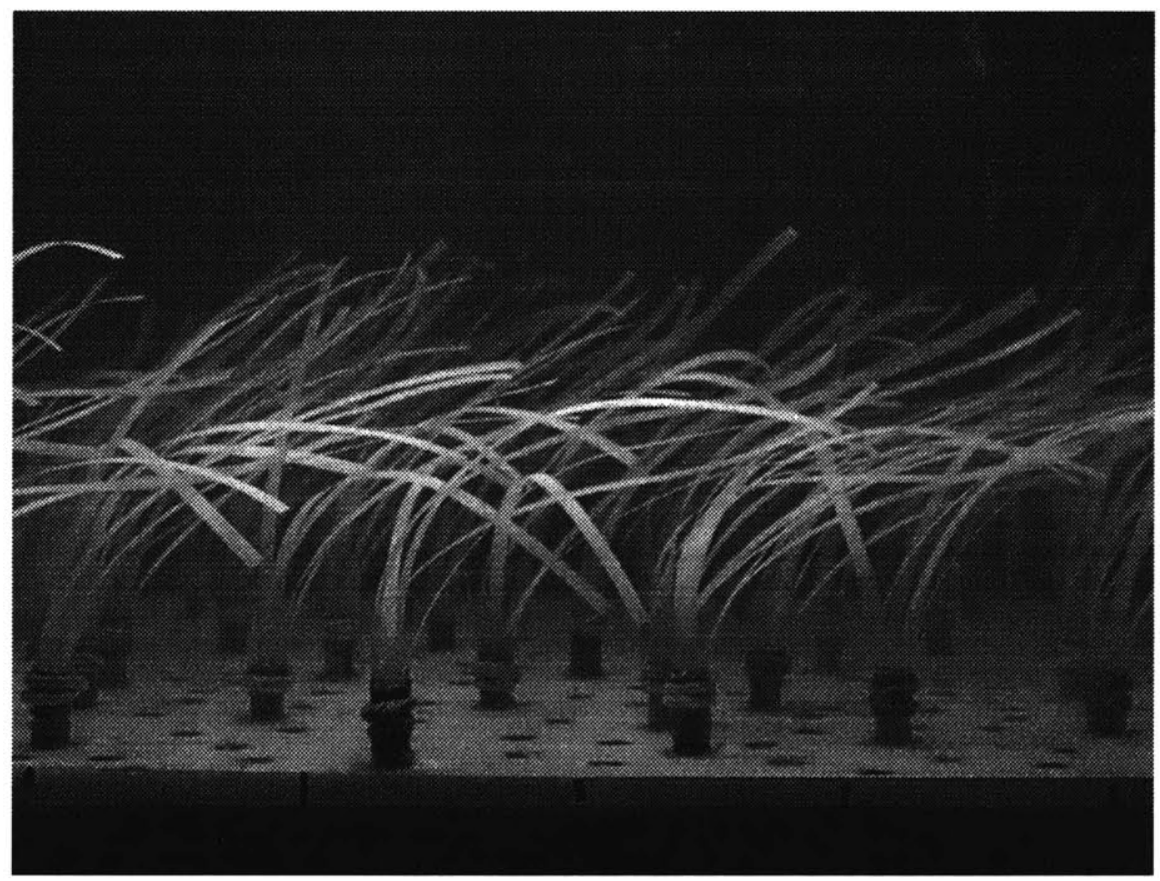

Figure 2.6. Photograph of the constructed model eelgrass meadow.

\subsection{Flow scenarios of the main experiments}

The main set of experiments performed in this study consisted of nine flow scenarios; each scenario distinguished from the others by changes in water depth $(H)$ and flowrate $(Q)$. The flow scenarios, detailed in Table 2.5, were chosen so as to be able to:

(i) observe conditions with and without the monami, and 
(ii) encompass a range of $H / h_{d}$ values, to observe the effect of decreasing surface layer depth on the monami.

\begin{tabular}{|c|c|c|c|c|c|}
\hline Scenario & $H(\mathbf{c m})$ & $Q( \pm \mathbf{5} \mathbf{g p m})$ & $\boldsymbol{h}_{\boldsymbol{d}}( \pm \mathbf{0 . 2} \mathbf{c m})$ & $\begin{array}{c}\text { Observable } \\
\text { monami } ?\end{array}$ & $\begin{array}{c}\text { Amplitude of } \\
\text { waving } \pm \mathbf{0 . 2} \mathbf{~ c m})\end{array}$ \\
\hline A & 35.9 & 176 & 8.5 & YES & 2.9 \\
\hline B & 35.9 & 73 & 11.3 & YES & 1.0 \\
\hline C & 35.9 & 20 & 12.9 & NO & - \\
\hline D & 29.0 & 155 & 6.4 & YES & 2.3 \\
\hline E & 29.0 & 63 & 9.4 & YES & 2.8 \\
\hline F & 29.0 & 12 & 12.7 & NO & - \\
\hline G & 16.4 & 76 & 6.2 & YES & 1.6 \\
\hline H & 16.4 & 17 & 9.4 & NO & - \\
\hline I & 12.3 & 20 & 9.0 & NO & - \\
\hline
\end{tabular}

Table 2.5. Flow and plant height parameters for the nine experimental runs comprising this study.

\subsubsection{Mean velocity profiles}

For each flow scenario, two sets of measurements were taken. The first data set consisted of vertical profiles of velocity records, taken using either the ADV or LDV as described in Chapter 2. All velocity records were taken at $x=6.5 \mathrm{~m}$, at heights separated vertically by approximately $1 \mathrm{~cm}$. Measurements could be taken to within $0.2 \mathrm{~cm}$ of the canopy bottom (with the ADV) and to within $0.5 \mathrm{~cm}$ of the free surface (LDV). For each scenario, a vertical profile was taken at three lateral positions in the flume, namely at $y=-3 \mathrm{~cm}, 0$ and +3 $\mathrm{cm}$. Measurement at 3 lateral positions, separated by a distance much greater than the lateral scale of an individual plant, was expected to encompass the lateral variability in the canopy. 
Note that analysis of lateral variability in mean and turbulent velocities by Vivoni (1998), above a model canopy in this flume, showed that the interior $20 \mathrm{~cm}$ were devoid of wall effects.

\subsubsection{Comparison of statistics obtained by the ADV and LDV}

Given that profiles of both mean and turbulent quantities incorporated data obtained by the ADV and by the LDV, it was hoped that the two forms of data would be consistent, if not identical. Using the two ADV and LDV records obtained at the same point during each run, empirical comparisons between the mean velocity measurements (generated using the MATLAB codes in Appendices A1 and A2) were obtained. The differences between the mean velocity estimates obtained by the two instruments were much larger than expected, and cause for concern. While Voulgaris and Trowbridge (1997) report differences of less than $1 \%$, results from this study showed much greater discrepancies (up to $1.2 \mathrm{cms}^{-1}$ and occasionally up to $30 \%$ ). The empirical relationship between the two estimates was:

$$
U_{A D V}=1.067 U_{L D V}+0.282
$$

where $U_{A D V}$ is the mean velocity estimate obtained by the ADV $\left(\mathrm{cms}^{-1}\right)$ and $U_{L D V}$ is the mean velocity estimate obtained by the $\operatorname{LDV}\left(\mathrm{cms}^{-1}\right)$. Fortunately, the relationship between the two estimates was consistent $\left(r^{2}=0.997\right)$. Therefore, all LDV estimates of mean velocity were 'converted' to ADV estimates using Equation 2.25.

As opposed to the agreement between ADV and LDV estimates of mean velocity, the agreement in the estimates of $r_{u w}$ (i.e. the coefficient of correlation between horizontal and vertical turbulent fluctuations) was neither good nor consistent. The discrepancy between the estimates varied between each flow scenario, ranging from $2 \%$ to $50 \%$. As a result, LDV correlation coefficient estimates were converted to ADV estimates using the mean ratio between the estimates obtained from the two records for that scenario only. Therefore, while 
correlation coefficient estimates from LDV records were indeed 'converted' to corresponding ADV estimates, little faith should be placed in them. 


\section{Chapter 3. Results and Discussion}

\subsection{Mathematical introduction}

Firstly, the mathematical description of turbulent fluid flow is presented here as a foundation for the following results and subsequent analysis. The Navier-Stokes equations, in tensor notation, for an incompressible, Newtonian fluid are as follows:

$$
\begin{gathered}
\frac{\partial u_{i}}{\partial x_{i}}=0 \\
\frac{D u_{i}}{D t}=-\frac{1}{\rho} \frac{\partial p}{\partial x_{i}}+g_{i}+\nu \nabla^{2} u_{i}
\end{gathered}
$$

where $x_{i}$ and $u_{i}$ represent the position and velocity vectors, $p$ the pressure, $\rho$ the fluid density, $v$ the kinematic viscosity and $g_{i}$ the gravitational acceleration vector. Substitution of the Reynolds decomposition of velocities and pressure (i.e. $\left.u_{i}=U_{i}+u_{i}^{\prime} ; p=P+p^{\prime}\right)$ into Equations 3.1 and 3.2 gives, respectively:

$$
\begin{gathered}
\frac{\partial U_{i}}{\partial x_{i}}=0 \\
\frac{\partial U_{i}}{\partial t}+U_{j} \frac{\partial U_{i}}{\partial x_{j}}+\frac{\partial \overline{u_{i}^{\prime} u_{j}^{\prime}}}{\partial x_{j}}=-\frac{1}{\rho} \frac{\partial P}{\partial x_{i}}+g_{i}+v \frac{\partial^{2} U_{i}}{\partial^{2} x_{j}}
\end{gathered}
$$

The spatial non-uniformity of local plant density and thus local velocity characteristics meant that lateral averaging of velocity statistics was required, as in Raupach and Shaw (1982). The lateral averaging of velocities and pressure is expressed as: 


$$
\begin{aligned}
& U_{i}=\left\langle U_{i}\right\rangle+U_{i}^{\prime \prime} \\
& p_{i}=\left\langle p_{i}\right\rangle+p_{i}^{\prime \prime}
\end{aligned}
$$

where the angular brackets denote the laterally-averaged quantity and the double primes the deviation from the average. For steady, two-dimensional $(x, z)$ flow through a seagrass meadow, lateral averaging yields the following relationships for vegetated flow, using the traditional parameterization of drag force:

$$
\begin{gathered}
\frac{\partial\langle U\rangle}{\partial x_{i}}+\frac{\partial\langle W\rangle}{\partial z}=0 \\
\langle U\rangle \frac{\partial\langle U\rangle}{\partial x}+\langle W\rangle \frac{\partial\langle U\rangle}{\partial z}=-\frac{1}{\rho} \frac{\partial\langle P\rangle}{\partial x}+\frac{1}{\rho} \frac{\partial\langle T\rangle}{\partial x}-\frac{1}{2} C_{D} a\langle U\rangle^{2}
\end{gathered}
$$

where (3.8) is simply the $x$-component of the series of equations in (3.4) and $T$ represents the temporal average of shear stress. Neglecting the viscous and dispersive stresses, as is valid in vegetated flows (Raupach and Shaw, 1982), the total stress is comprised solely of the Reynolds stress:

$$
T=-\rho \overline{u^{\prime} w^{\prime}}
$$

Thus, changes in streamwise momentum are governed by the driving pressure force (due to the setup of a surface slope above the model canopy), the imposed drag force due to the vegetation and Reynolds stress. 


\subsection{Preliminary test}

As a preliminary test, the evolution of the vertical profile of the temporal mean of longitudinal velocity, $U$, along the model eelgrass meadow was examined. This test was performed for two reasons:

(a) to indicate where in the canopy the flow had become fully developed, thereby providing a reasonable place to perform the main experiments, and

(b) to examine the vertical profile of $U$ immediately upstream of the canopy. It was hoped that the modification to the inlet conditions (shown in Figure 2.1) would result in the development of a typical turbulent boundary layer velocity profile by the time the flow encountered the meadow. The immediate aim was the prevention of a discharge jet, whereby a region of anomalously high velocity exists at the height of the upstream inlet.

The flume was filled to a depth of $41.0 \mathrm{~cm}$ with a flowrate of $166 \pm 3 \mathrm{gpm}$, providing an intermediate depth-averaged velocity of $6.7 \mathrm{cms}^{-1}$. Six vertical profiles of mean velocity were taken in the center of the flume at $x=-0.6 \mathrm{~m}, 0.5 \mathrm{~m}, 2.2 \mathrm{~m}, 3.5 \mathrm{~m}, 5.0 \mathrm{~m}$ and $6.5 \mathrm{~m}$. For each vertical profile, five minute velocity records were taken with the ADV, at locations separated vertically by $1 \mathrm{~cm}$. As can be seen from Figure 3.1, the shear layer never extended up to the $7 \mathrm{~cm}$ region immediately beneath the free surface; consequently, the LDV was not employed. Note that although $h_{d}$ is a weak function of $x$ (due to the longitudinal variation of the mean velocities to which the plants are exposed), the average value of $h_{d}$ was approximately $8.8 \mathrm{~cm}$. 


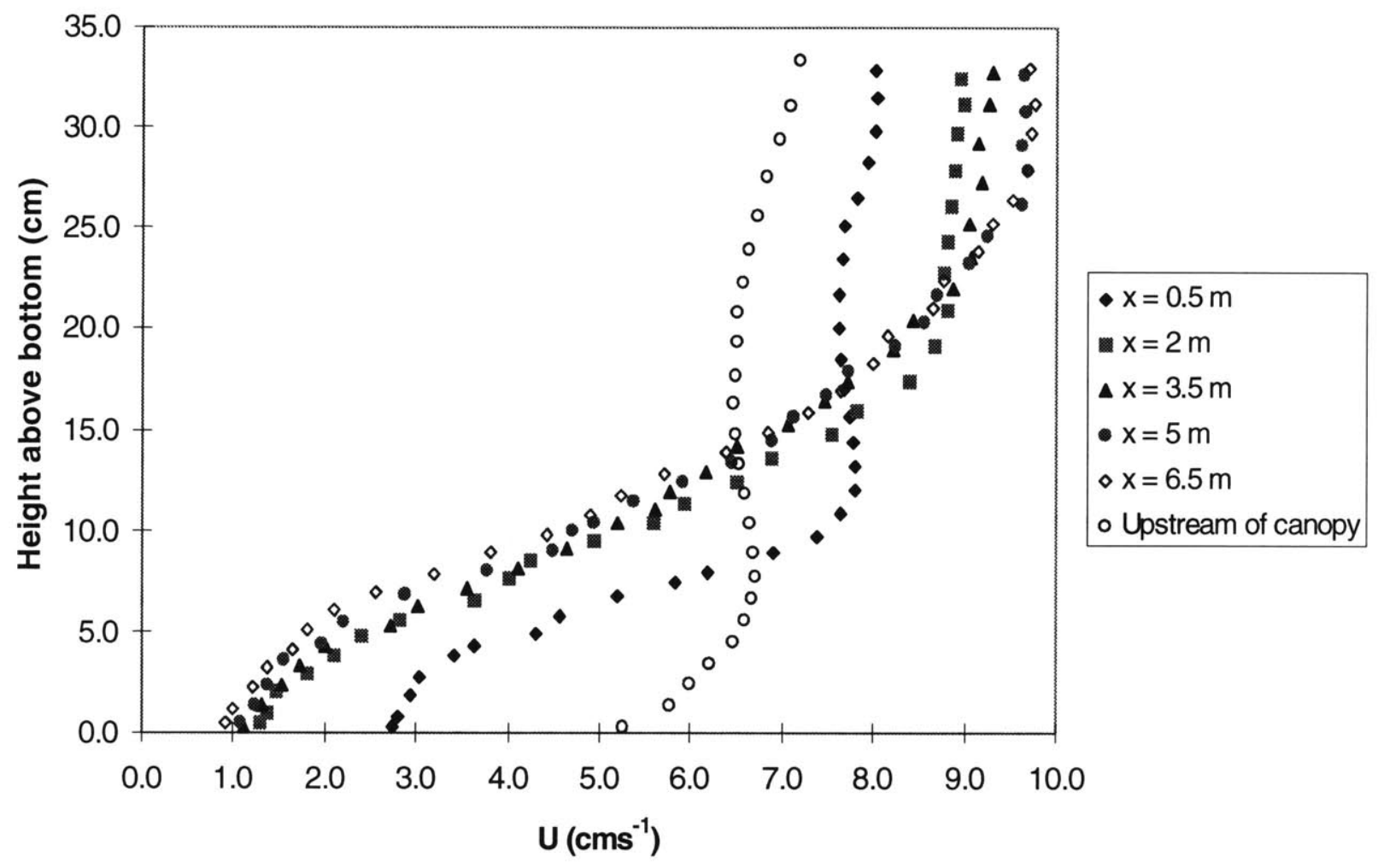

Figure 3.1. Evolution of the vertical profile of mean longitudinal velocity as the flow encounters the model eelgrass meadow.

The velocity profile immediately upstream of the canopy indicates that the prevention of a discharge jet had been moderately successful, although the profile still differed significantly from that of fully developed turbulent flow in an open channel. However, the memory of upstream conditions is clearly lost once uniform flow is attained within the canopy. The drag exerted by the canopy causes a spatial deceleration of the fluid near the bottom. By continuity, the overlying fluid must undergo a spatial acceleration, associated with the partial redirection of the flow over the top of the meadow. This leads to the creation of a region of strong shear, characterized by the presence of an inflection point.

Figure 3.1 demonstrates that approximately uniform flow conditions had been reached well before the end of the canopy, with little development of the velocity profile beyond 
$x=5 \mathrm{~m}$. Consequently, $x=6.5 \mathrm{~m}$ was deemed to be an appropriate sampling point for future experiments as it was expected to be representative of uniform in-canopy conditions in the field.

Figure 3.2 shows the development of $\vec{U}, \Delta U$ and $\theta$ as the flow progresses into the canopy. The results are in stark contrast to the typical mixing layer generated in the laboratory (i.e. that between two free streams separated initially by a splitter-plate). Such a mixing layer has a constant, prescribed value of $\Delta U$ and a momentum thickness that increases continually, by diffusion of momentum, until boundaries prevent further development. In this case, $\Delta U$ is initially zero and grows similarly to $\theta$ as the (vertically non-uniform) vegetation drag is exerted on the flow. In addition, the momentum thickness tapers off even though the mixing layer never fully extends to the free surface. The absence of continued mixing layer growth is further discussed in Section 3.2.1. 


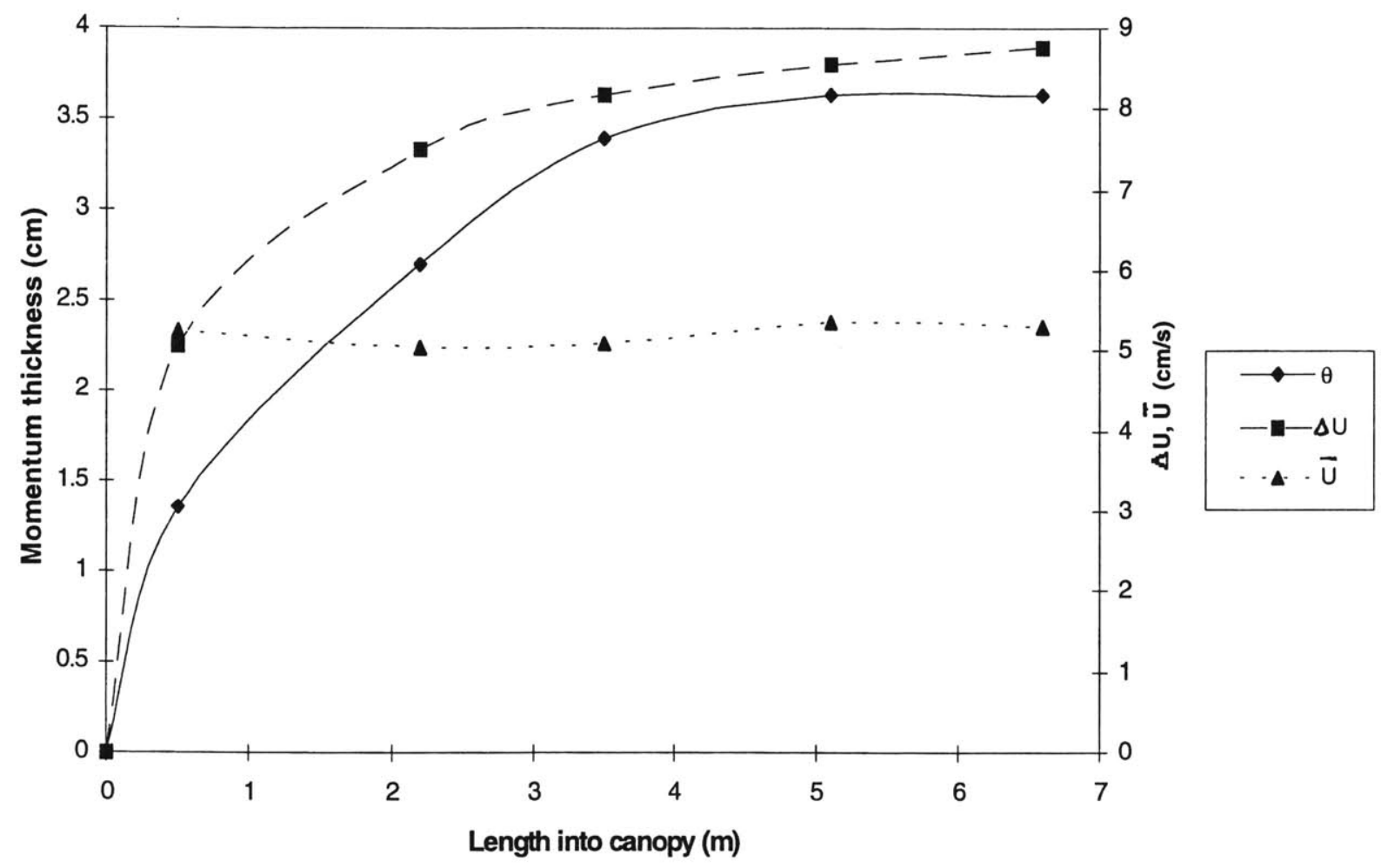

Figure 3.2. Development of three fundamental mixing layer parameters as the flow progresses into the model canopy.

The coherent waving (monami) of the plants was observed to begin approximately $0.7 \mathrm{~m}$ into the canopy and became more pronounced as it progressed along the canopy. By the end of the canopy, the vertical excursion of the plants during their waving cycle was approximately $3.0 \mathrm{~cm}$.

The monami was separated into three streamwise channels. Each channel underwent the cycle of coherent, progressive waving but was noticeably out of phase with the other channels, such that at any point in time, the deflected plant height exhibited significant variability across the model canopy. This is in agreement with Ikeda and Kanazawa (1996) and Vivoni (1998) who estimated the lateral scale of the coherent vortices generated above a plant 
canopy to be of the order $1.5-3 h_{d}$. The flume in question is $38 \mathrm{~cm}$ wide, giving a monami channel width of $12.7 \mathrm{~cm}$ (or $\left.\sim 1.4 h_{d}\right)$.

\subsubsection{Presence of secondary circulation}

As previously noted, the momentum thickness of mixing layers grows continually in the absence of boundary effects. Our observation of the momentum thickness growing asymptotically is therefore somewhat anomalous, although the same phenomenon can be seen in the flume experiments of Gambi et al. (1990). The roughness created by the vegetation, and the comparative smoothness of the flume walls, can set up outward lateral currents. This is known as differential roughness secondary flow (DRSF) and is discussed further in Pantin et al. (1981). The presence of the sidewalls can therefore result in secondary circulation (see Figure 3.3 (a)).

To check for the presence of strong secondary circulation in the flume, two lateral transects of ten minute velocity records were taken with the ADV at $z=h_{d}(\sim 8.8 \mathrm{~cm})$. Records were taken every $3 \mathrm{~cm}$ in the lateral direction and the transects were taken at $x=2.5 \mathrm{~m}$ and $x=4.0 \mathrm{~m}$. Special care was taken to straighten the probe before the records were taken, to avoid incorporating any component of the longitudinal velocity into the measured lateral velocities. Nevertheless, at the completion of both transects, a velocity record was taken upstream of the canopy to determine the tilt in the probe; the lateral and vertical tilt angles were both less than $0.2^{\circ}$. The secondary flow in the flume is shown in Figure 3.3 (b). Note that this figure indicates the presence of outward lateral flow on both sides, as one would expect. 
(a)

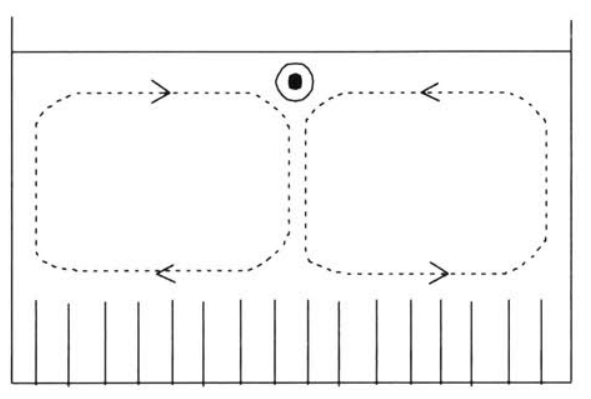

(b)

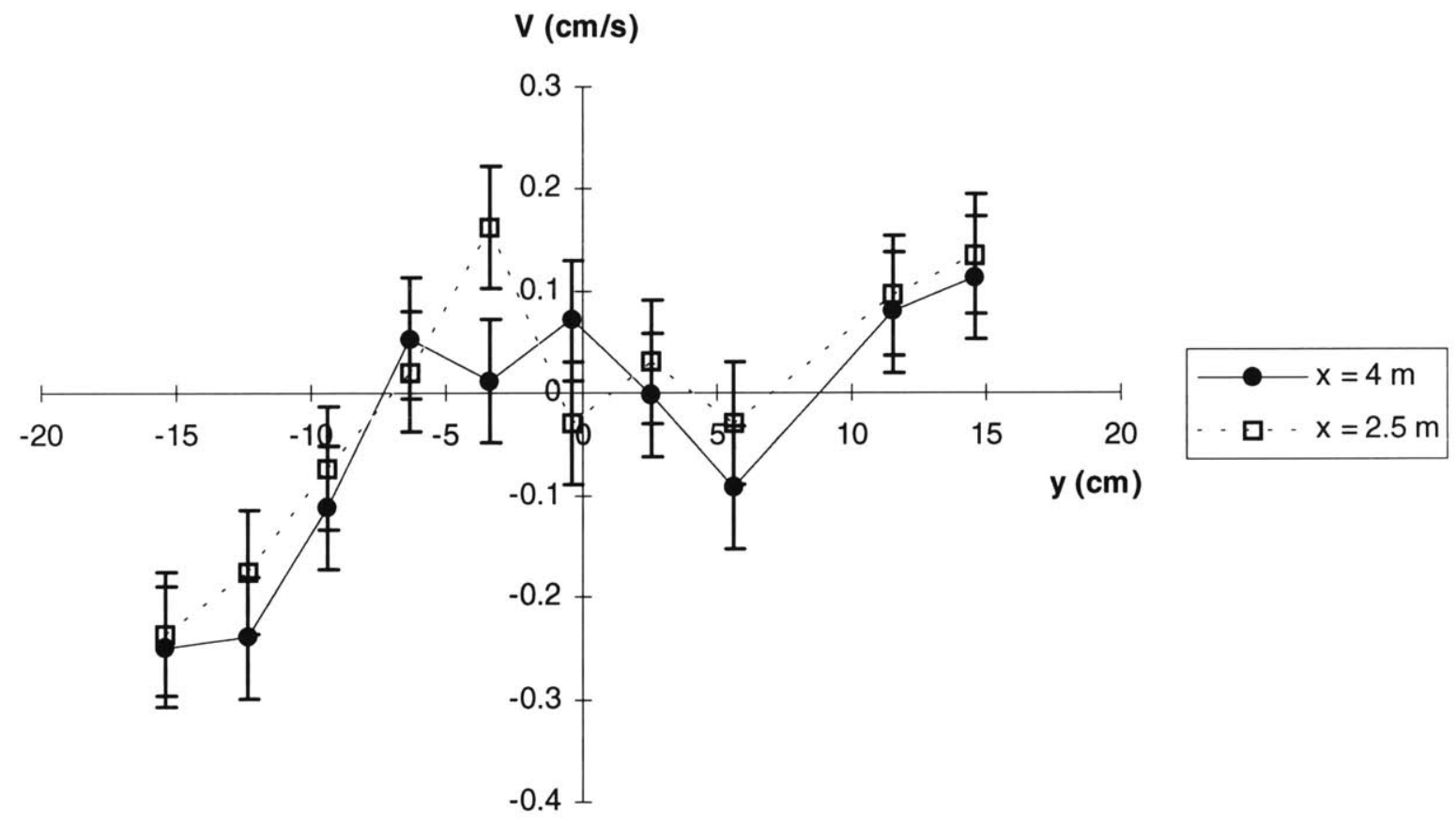

Figure 3.3 (a) Schematic diagram of expected secondary circulation above model vegetation.

(b) Evidence of mild secondary circulation generated in the flume. Vertical bars represent uncertainty in $V$ based on the estimated uncertainty in determining the tilt of the probe $\left( \pm 0.1^{o}\right)$.

Taking an estimated circulation velocity of $0.2 \mathrm{cms}^{-1}$, a water particle would take $\sim 510$ s to complete one circulation; this is equivalent to the time taken for the flow to progress 
more than $30 \mathrm{~m}$ into the canopy. Given that the mean velocity profile changes very little after $x=5 \mathrm{~m}$, it seems unlikely that the induced secondary circulation accounts fully for the asymptotic behavior of the profile.

\subsection{Triggering the monami phenomenon}

Preliminary visual observations of the model eelgrass meadow and the field observations of Grizzle et al. (1996) indicated that the coherent waving of the plants was not present under all flow conditions. From six observations, the authors indeed observed the presence of the monami only once, under maximum flow depth and flow velocity. Similarly, Fonseca and Kenworthy (1987) indicated that the monami generally occurs at higher flow velocities. Consequently, a simple experiment was undertaken to determine the flow conditions required in the flume for the observation of coherent waving.

The flume was filled to a water depth of $41 \mathrm{~cm}$ and the valve was opened up fully, such that the pump was operating at its maximum flowrate. The monami phenomenon was clearly present under these conditions. The valve was then progressively closed until the coherent waving could no longer be observed. At this point, the flow velocity at the canopy-water interface $\left(U_{h}\right)$ was measured with the LDV. The transition from an observable monami to a lack thereof was surprisingly rapid and consistent; the reverse transition always occurring at the same flowrate. The flume was then drained of $2 \mathrm{~cm}$ of water and the entire process repeated. For every water depth, the monami was observed at the highest flowrate and disappeared as the mean flow speed decreased. The interface velocities required to trigger the monami, along with the $H / h_{d}$ ratio at that critical value of $U_{h}$ at the various flow depths are shown in Figure 3.4. In this figure, the domain of depth and/or velocity greater than the dashed line represents where the monami is present. 


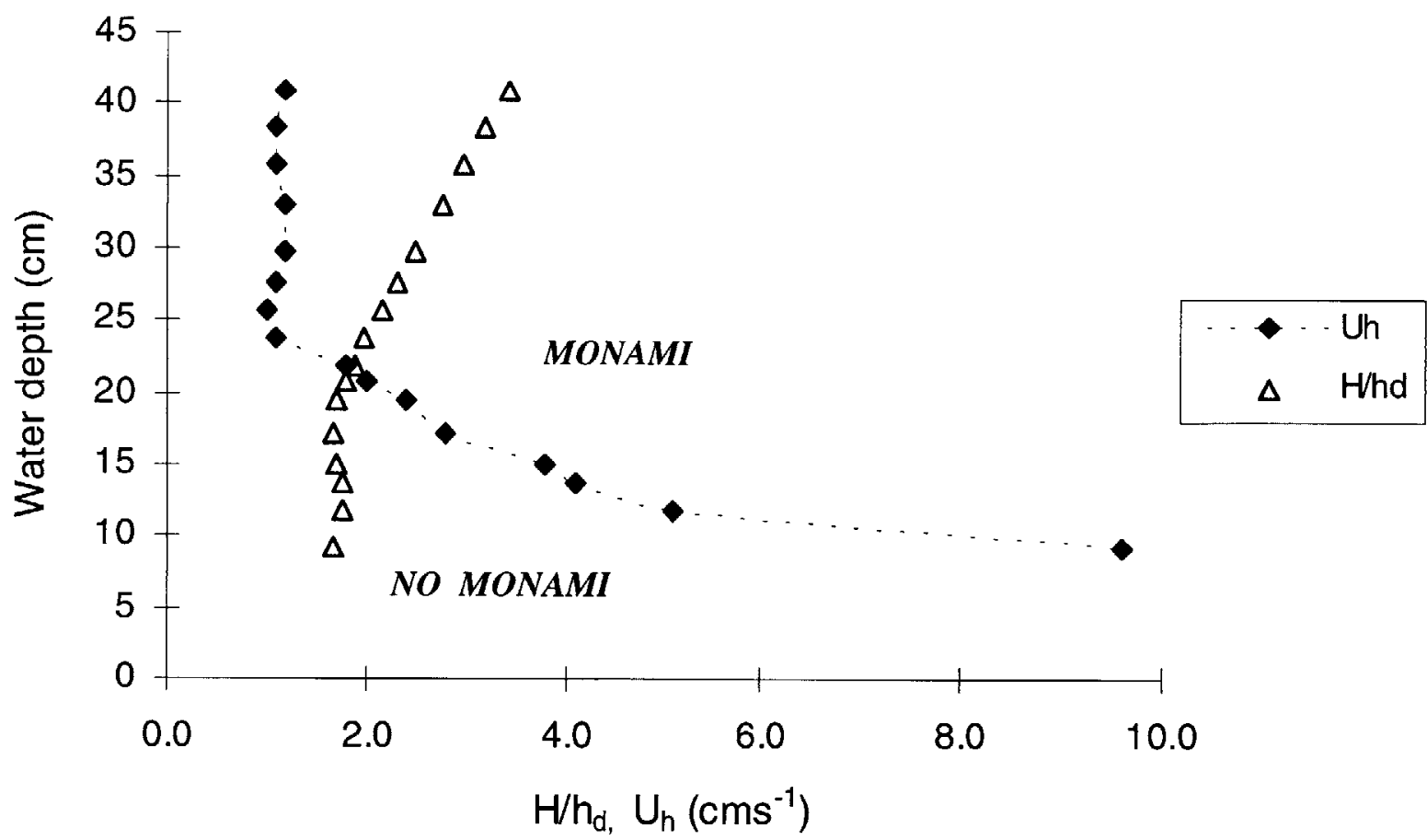

Figure 3.4. The conditions required for observation of the monami in the laboratory flume. The dashed line represents the transition from the monami to a lack thereof.

The curve shown in Figure 3.4 consists of two regions; water depths where the interface velocity required to trigger the monami is constant and shallower depths where the trigger velocity increases as the depth is reduced. It should be stressed that the relationship shown in Figure 3.4 is valid only for our specific experimental model of an eelgrass meadow. Changes in plant density, dimensions and stiffness would undoubtedly result in a different curve; the emphasis here is on the qualitative nature of the curve. Indeed, Grizzle et al. (1996) speculated upon a trigger interface velocity of $10 \mathrm{cms}^{-1}$ for real eelgrass meadows when $H / h_{d}$ was approximately 2 ; at this $H / h_{d}$ value, the observed trigger velocity in the flume was $1.2 \mathrm{cms}^{-1}$. This is discussed further in Section 3.5.1. 


\subsection{The appropriateness of the mixing layer analogy}

Reassuringly, the presence (or lack thereof) of the monami phenomenon in all flow scenarios agreed with expectation, based on Figure 3.4. The final column of Table 2.5 details the maximum vertical excursion exhibited by the plants whilst waving, estimated from the video footage; clearly, the monami phenomenon can be very pronounced, given that the waving excursion of the plants is up to $35 \%$ of the mean plant height.

The initial, and most obvious, means of comparison between flow through submerged vegetation and that of a mixing layer lies in the examination of the mean velocity profile. The mean (laterally averaged) velocity profiles of these experiments are shown in Figure 3.5.

(a)

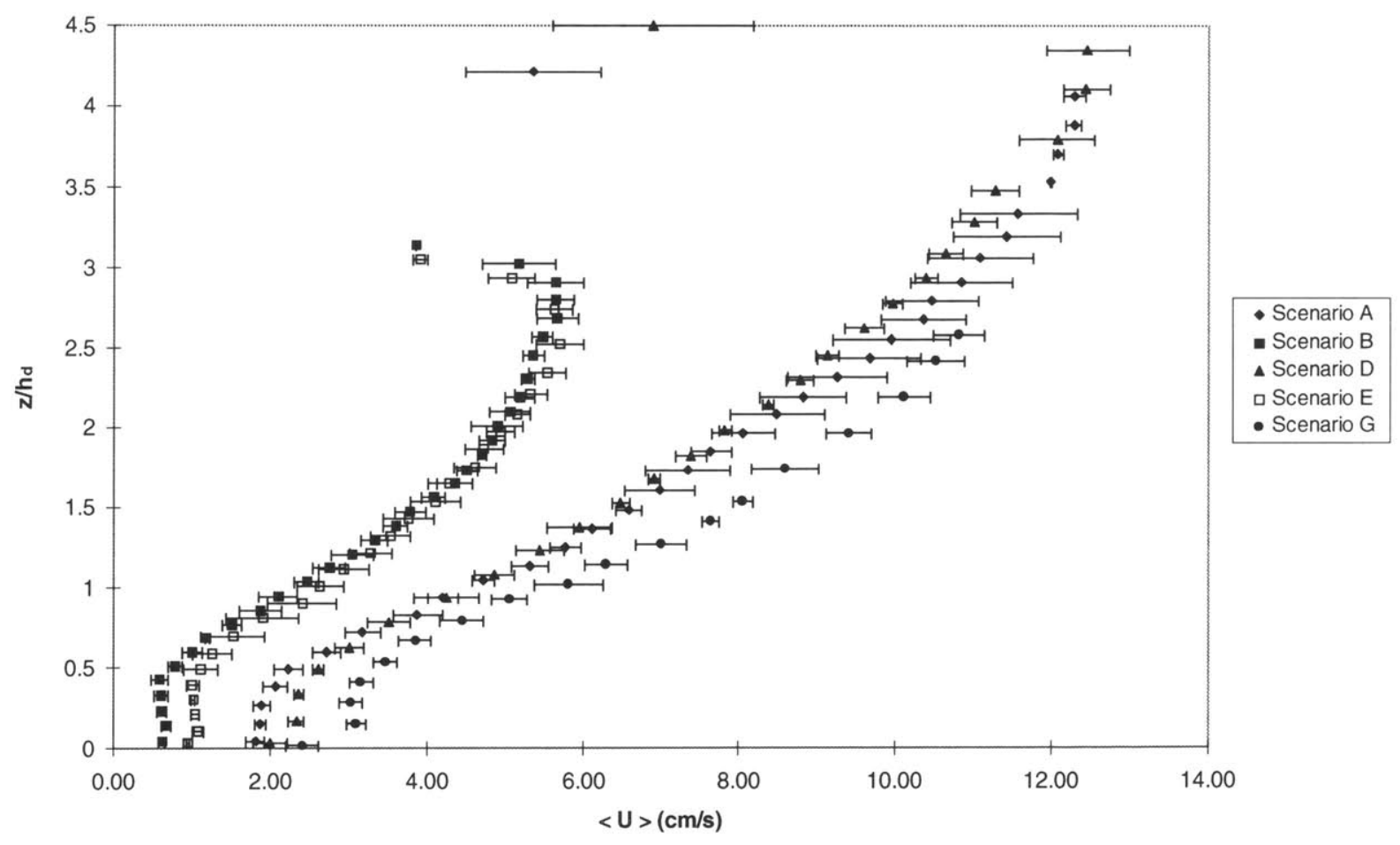


(b)

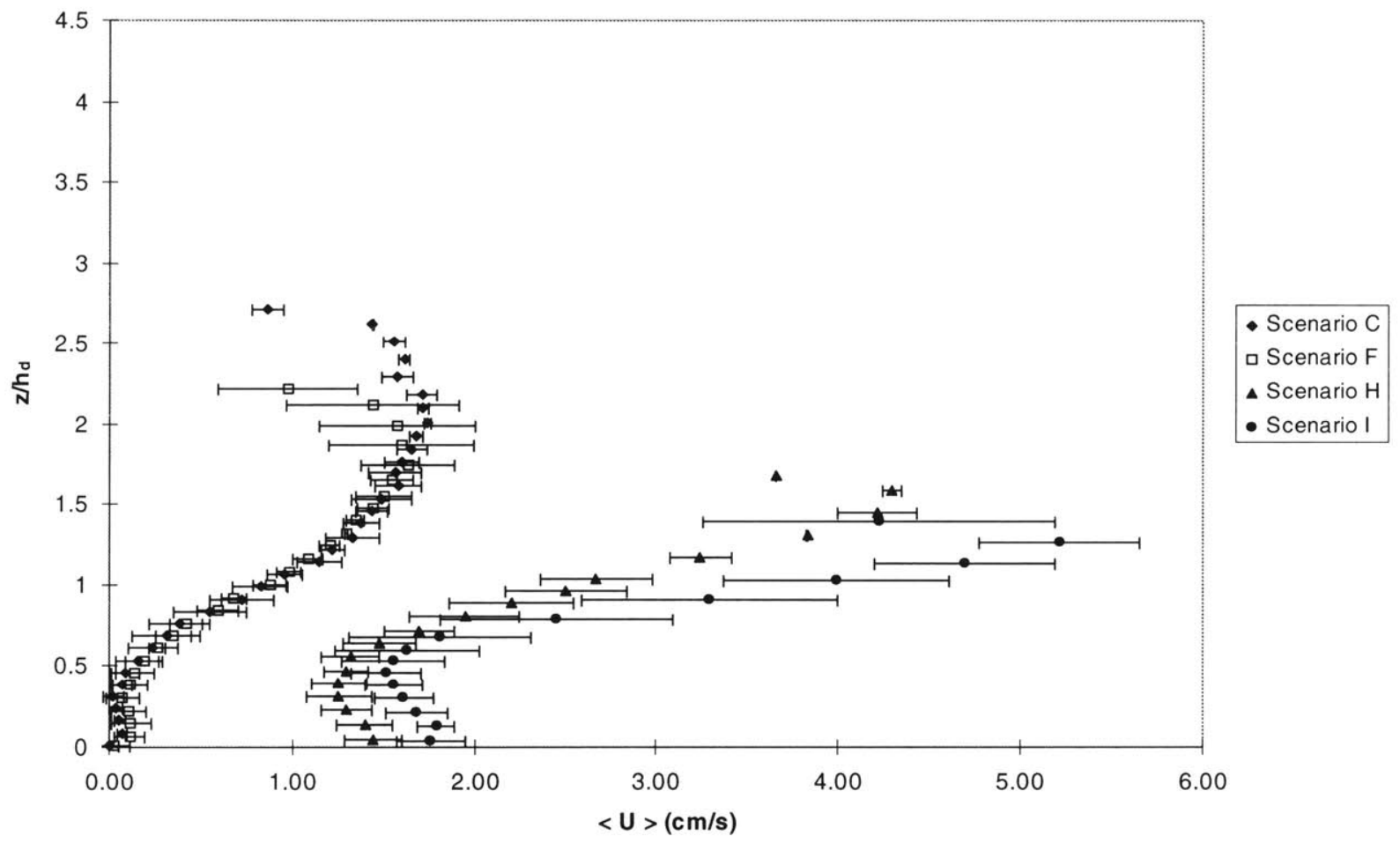

Figure 3.5. (a) Laterally averaged mean velocity profiles for flow scenarios with the monami.

(b) Laterally averaged mean velocity profiles for flow scenarios without an observable monami. In both cases, horizontal bars indicate the standard deviation of the measurements at multiple lateral locations.

The mean velocity profiles in Figure 3.5 demonstrate the strong mixing layer nature of the flow, with some peculiarities particular to open channel flow through submerged vegetation. Particularly in the case where there exists no monami, there appears to be a region of reverse shear (i.e. where the velocity increases as the bed is approached) in the lower half of the canopy. In addition, in the region near the free surface there is a reduction in streamwise velocity, as is typical in open-channel flow. 
All velocity profiles, however, have strong mixing layer characteristics. This is further exemplified by Figure 3.6, in which the height and velocity axes of the mean velocity profiles are shifted by their average values $(\bar{z}$ and $\bar{U}$, as defined in Section 1.1.3) and normalized by $\theta$ and $\Delta U$ respectively. The comparison to the traditional hyperbolic tangent profile of a mixing layer, namely:

$$
\frac{z-\bar{z}}{\theta}=0.5 \times \tanh \left(\frac{U-\bar{U}}{2 \Delta U}\right)
$$

is favorable, with some slight yet important differences.

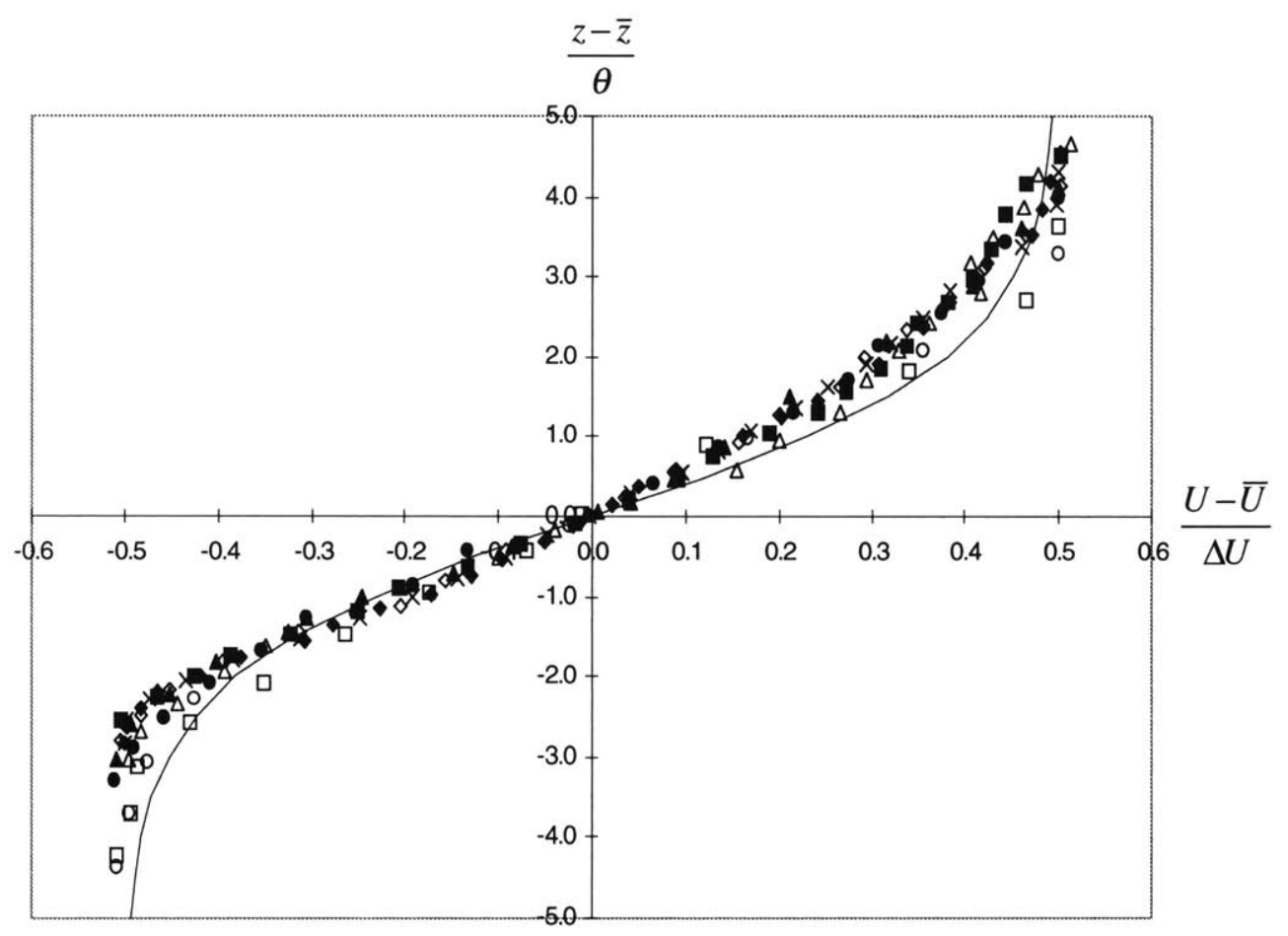

- Scenario A

- Scenario $B$

$\triangle$ Scenario $C$

$\times$ Scenario $D$

$\diamond$ Scenario $E$

- Scenario $F$

- Scenario G

口 Scenario $\mathrm{H}$

- Scenariol

Hyperbolic tangent

Figure 3.6. Comparison between the observed velocity profiles and the traditional hyperbolic tangent profile of a mixing layer. 


\subsubsection{Quantification of the mixing layer analogy}

In accordance with the mixing layer analogy that forms the basis of this study, several parameters associated with pure mixing layers can be estimated for vegetated flow. Specifically, the parameters introduced in Table 3.1 are:

- $R=\frac{\Delta U}{2 \bar{U}}$. This quantity represents the overall shear in the mixing layer, and influences several aspects of mixing layer behavior, such as the growth rate (Brown and Roshko, 1974; Ho and Huerre, 1984; Rogers and Moser, 1994).

- $R e_{h m}$ - the mixing layer thickness Reynolds number, defined by:

$$
\operatorname{Re}_{h m}=\frac{\Delta U h_{m}}{v}
$$

This parameter governs the transition of mixing layers from laminar to turbulent; although the coherent vortices are present in both, this transition is accompanied by a dramatic increase in small-scale mixing (Moser and Rogers, 1991). 


\begin{tabular}{|c|c|c|c|c|c|c|c|c|}
\hline Scenario & $\begin{array}{c}\bar{U} \\
\left(\mathbf{c m s}^{-1}\right)\end{array}$ & $\begin{array}{c}\Delta U \\
\left(\mathbf{c m s}^{-1}\right)\end{array}$ & $R$ & $\theta(\mathbf{c m})$ & $h_{m}(\mathbf{c m})$ & $h_{m} / h_{d}$ & $h_{m} / \theta$ & $R e_{h m}$ \\
\hline A & 7.12 & 10.49 & 0.74 & 4.51 & 32.2 & 3.79 & 7.1 & 33800 \\
\hline B & 3.14 & 5.03 & 0.80 & 3.62 & 25.5 & 2.26 & 7.0 & 12800 \\
\hline C & 0.89 & 1.66 & 0.93 & 2.71 & 19.9 & 1.54 & 7.3 & 3300 \\
\hline D & 7.40 & 10.12 & 0.68 & 3.74 & 25.6 & 4.00 & 6.8 & 25900 \\
\hline E & 3.37 & 4.66 & 0.69 & 2.90 & 20.0 & 2.13 & 6.9 & 9320 \\
\hline F & 0.88 & 1.51 & 0.86 & 2.38 & 17.3 & 1.36 & 7.3 & 2600 \\
\hline G & 6.96 & 7.71 & 0.55 & 2.00 & 13.8 & 2.23 & 6.9 & 10640 \\
\hline H & 2.70 & 2.84 & 0.53 & 1.43 & 11.2 & 1.19 & 7.8 & 3180 \\
\hline I & 3.38 & 3.68 & 0.54 & 0.91 & 5.8 & 0.64 & 6.4 & 2130 \\
\hline
\end{tabular}

Table 3.1. Important mixing layer parameters of the mean velocity profile for all flow scenarios.

The consistency of the $h_{m} / \theta$ ratio $(7.1 \pm 0.4)$ is useful, as it provides a relatively simple means of estimating the momentum thickness without the requirement of obtaining a complete velocity profile.

Given that all profiles were taken at $x=6.5 \mathrm{~m}$, the value of $h_{m}$ in Table 3.1 is indicative of the spatial growth rate of the generated mixing layer. The inverse relationship between $R$ and the mixing layer growth rate (e.g. Rogers and Moser, 1994), is therefore apparent from Table 3.1 when comparing scenarios of the largest flow depth (e.g. Scenarios A-C). The complicating effects of the free surface, which may restrict mixing layer growth, limit the correlation between the two parameters, however. The magnitude of $\Delta U$ (and hence the value of $R$ ) is a function of the exerted vegetation drag (which in turn scales upon the square of the mean 
velocity) and the flow depth. Unlike in the typical splitter-plate experiments, $R$ can not be prescribed a priori in vegetated flow, and indeed it changes along the length of the canopy (Figure 3.2).

\subsubsection{Important vertical length scales}

With reference to Figure 3.6, the point of inflection of the experimental mean velocity profiles is not at $z=\bar{z}$, but is situated significantly below that point; this is further demonstrated in Table 3.2. In this table, four important vertical length scales in the mixing layer analogy of flow through aquatic vegetation are compared:

- $h_{d}$, the deflected height of the vegetation.

- $\bar{z}$, the height above the bottom of the center of the mixing layer,

- $z_{i n f l}$, the height at which the inflection point in the velocity profile is situated (i.e. where $d U / d z$ is maximized). This was estimated from the values of $\Delta U / \Delta z$ between each data point in the profile. In profiles where a single, clear maximum was not present, a threepoint moving average was applied to identify the inflection point.

- $z_{\text {wrms }}$, the height at which the standard deviation of the vertical velocity record (i.e. the vertical turbulent velocity, $w_{r m s}$ ) reached its maximum value. This was assumed to be the height of the central axis of vortex propagation, as one would expect $w^{\prime}$ to be maximized along the central longitudinal axis of an elliptical vortex. Vertical profiles of $w_{r m s}$, normalized by the mean mixing layer velocity, $\bar{U}$, for Scenarios $\mathrm{A}$ and $\mathrm{C}$ are shown in Figure 3.7 (ADV data only). The asymmetry of the profiles about $z_{w r m s}$ is indicative of a reduced kinematic influence of the vortices within the canopy; this is discussed further in Section 3.5.3. 


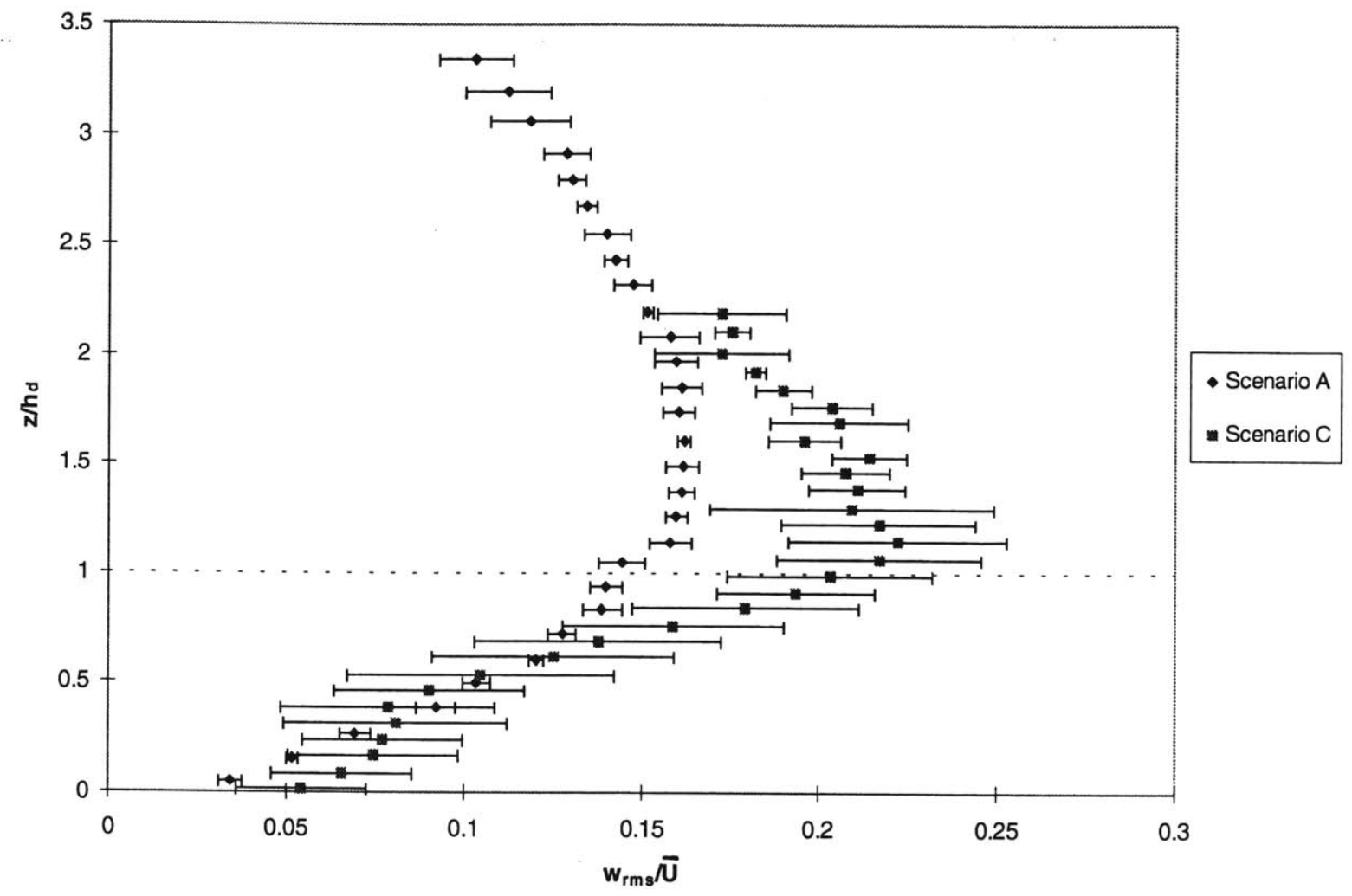

Figure 3.7. Vertical profiles of $w_{r m s}$ (normalized by $\bar{U}$ ) for Scenarios $A$ (monami) and $C$ (no monami). The dashed line indicates the deflected height of the vegetation.

As demonstrated by Table 3.2, the inflection points of the velocity profiles lie at or just below the canopy-water interface $\left(z_{\text {inf }} / h_{d}=0.95 \pm 0.08\right)$, in accordance with the findings of Vivoni (1998). In fact, the location of the inflection point may be a better indication of the effective height of the canopy, as indicated by Raupach et al. (1996), rather than a temporally and spatially averaged visual estimate. The relationship between $z_{\text {wrms }}$ and $\bar{z}$ also suggests that the vortices are essentially located in the center of the mixing layer $\left(z_{w r m} d \bar{z}=1.07 \pm 0.08\right)$. However, the center of the mixing layer lies well above the inflection point $\left(\bar{z} / z_{\text {inf }}=1.31 \pm\right.$ 0.29 ), which is in stark contrast to the typical mixing layer profile which is symmetrical about $\bar{z}$. This concurs with the flow visualization findings of Ikeda and Kanazawa (1996) and is symptomatic of the redirection of flow over the top of the canopy. 


\begin{tabular}{|c|c|c|c|c|}
\hline Scenario & $h_{d}(\mathbf{c m})$ & $\bar{z}(\mathbf{c m})$ & $z_{\text {infl }}(\mathrm{cm})$ & $z_{\text {wrms }}(\mathrm{cm})$ \\
\hline $\mathrm{A}$ & 8.5 & 14.1 & 9.1 & 13.7 \\
\hline $\mathrm{B}$ & 11.3 & 14.0 & 11.9 & 14.7 \\
\hline $\mathrm{C}$ & 12.9 & 13.2 & 11.3 & 15.8 \\
\hline $\mathrm{D}$ & 6.4 & 11.7 & 6.0 & 11.7 \\
\hline $\mathrm{E}$ & 9.4 & 11.8 & 8.5 & 13.4 \\
\hline $\mathrm{F}$ & 12.7 & 12.7 & 12.1 & 12.7 \\
\hline $\mathrm{G}$ & 6.2 & 7.8 & 6.0 & 9.1 \\
\hline $\mathrm{H}$ & 9.4 & 9.7 & 8.7 & 9.8 \\
\hline $\mathrm{I}$ & 9.0 & 7.9 & 7.5 & 8.8 \\
\hline
\end{tabular}

Table 3.2. Contrast between the vertical length scales of the mixing layer flow and plant canopy.

In the initial stages of flow development, the inflection point, canopy height and mixing layer center all correspond to the one height (Figure 3.1). As the flow is redirected over the canopy, the mixing layer continues to evolve, but at an increasingly greater height. The region of maximum shear is always fixed at the point where there is a transition from exerted drag to a lack thereof (i.e. at the top of the canopy).

\subsubsection{Comparison of frequency spectra and monami frequencies}

As observed by several researchers (Ackerman and Okubo, 1993; Grizzle et al., 1996; Wallace et al., 1998), the spectrum of streamwise velocity exhibits a strong, characteristic peak at a frequency that is invariant over depth. All records of longitudinal velocity in this study were resampled at a frequency $\left(f_{r}\right)$ between 1 and $4 \mathrm{~Hz}\left(\gg f_{K H}\right)$. These values of $f_{r}$ were 
sufficiently large such that the characteristic peaks in the spectra were observable and sufficiently small such that computational time was significantly reduced. The autocorrelation function of the resampled record was then calculated and smoothed using a Parzen window of width $\left(w_{w}\right)$ of between $40 f_{r}$ and $60 f_{r}$. From the smoothed autocorrelation function, the power spectrum of longitudinal velocity was obtained (Appendix B). In the power spectra for each flow scenario, peaks of an invariant frequency were observed throughout the generated mixing layer. Not surprisingly, identification of spectral peaks was easier for the flows with lower values of $R e_{h m}$, where there is a lack of small-scale turbulence superimposed upon the coherent structures that we are attempting to identify.

The strong peaks in the spectra of longitudinal velocity, and the constancy of the peak frequency over depth, are demonstrated in Figure 3.8. 
(i)

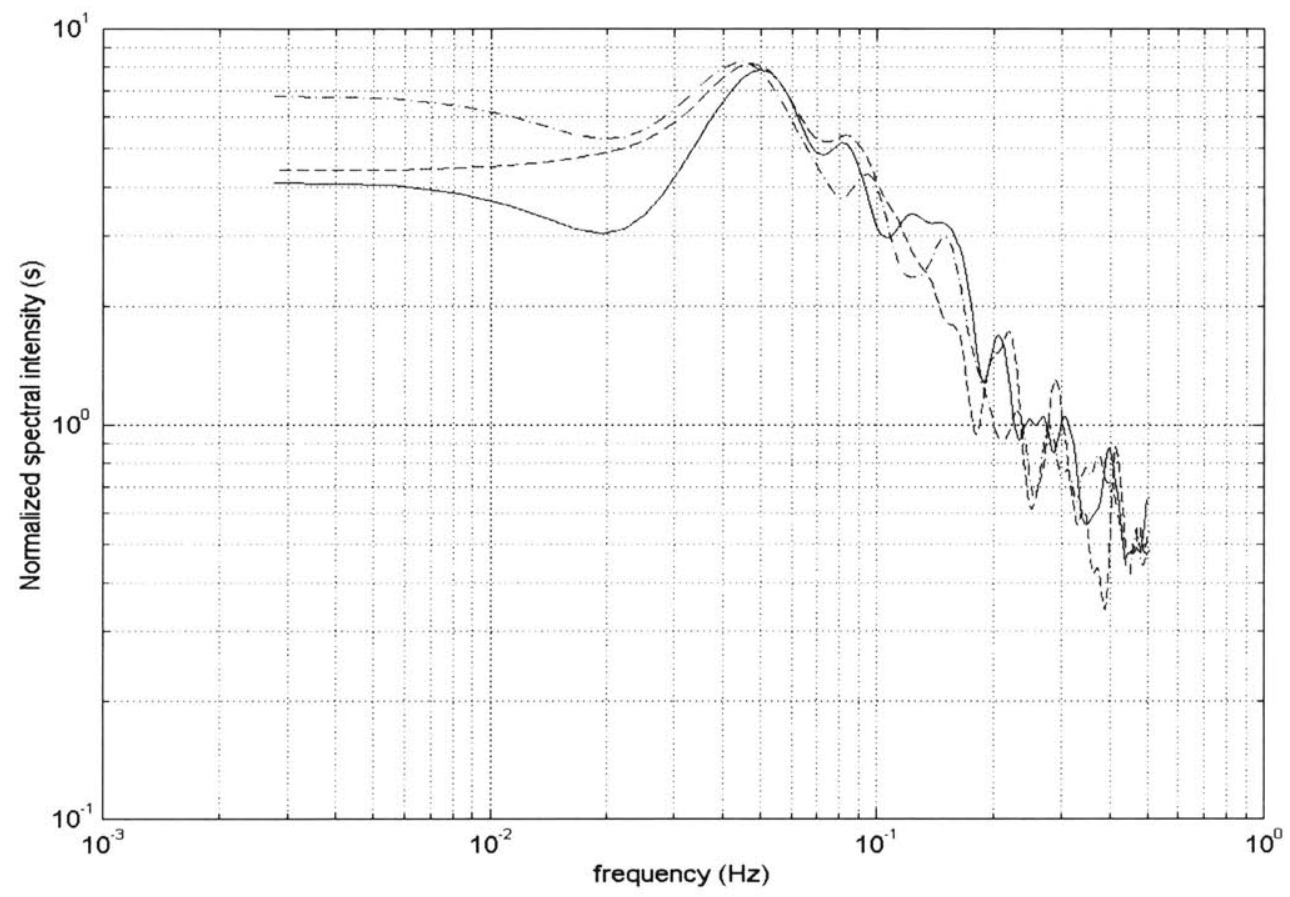

(ii)

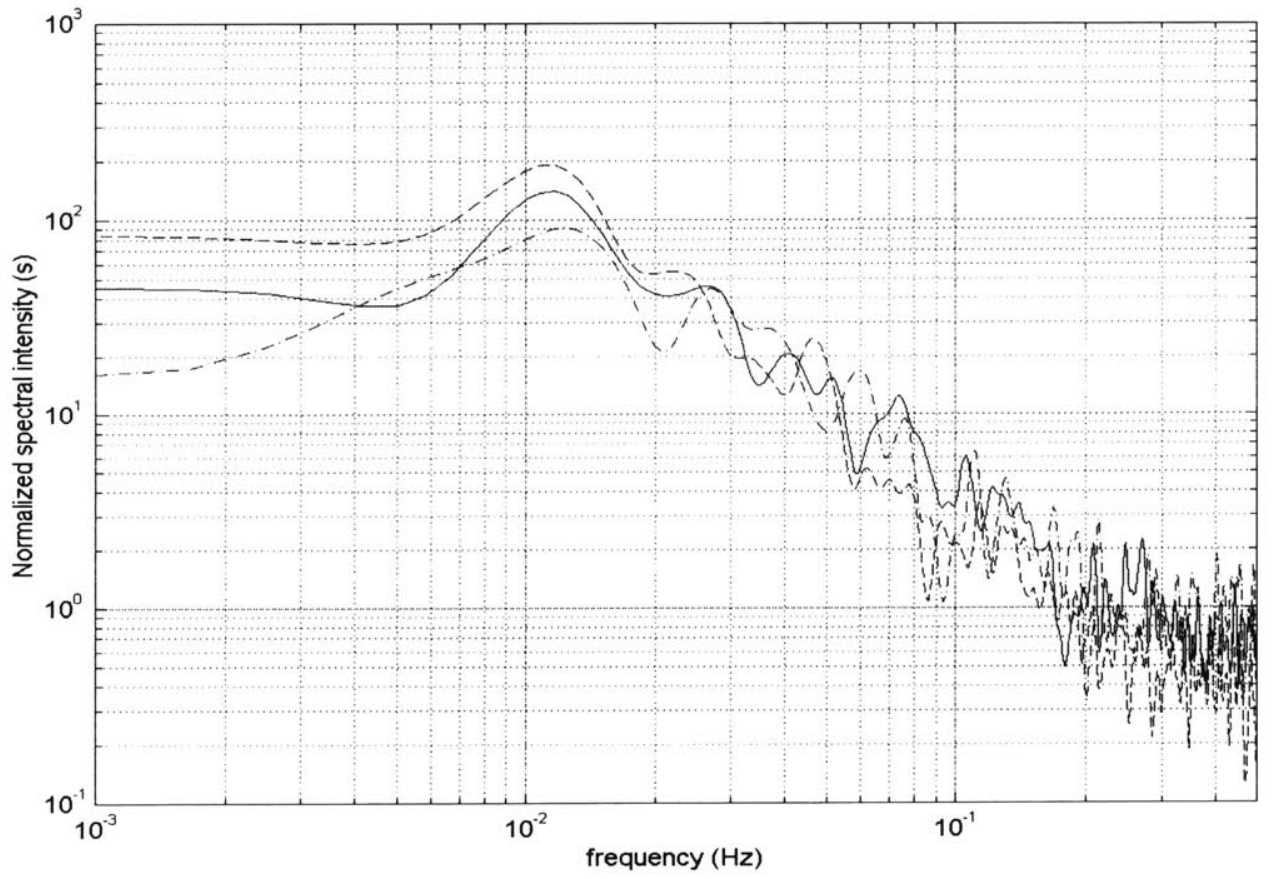


(iii)

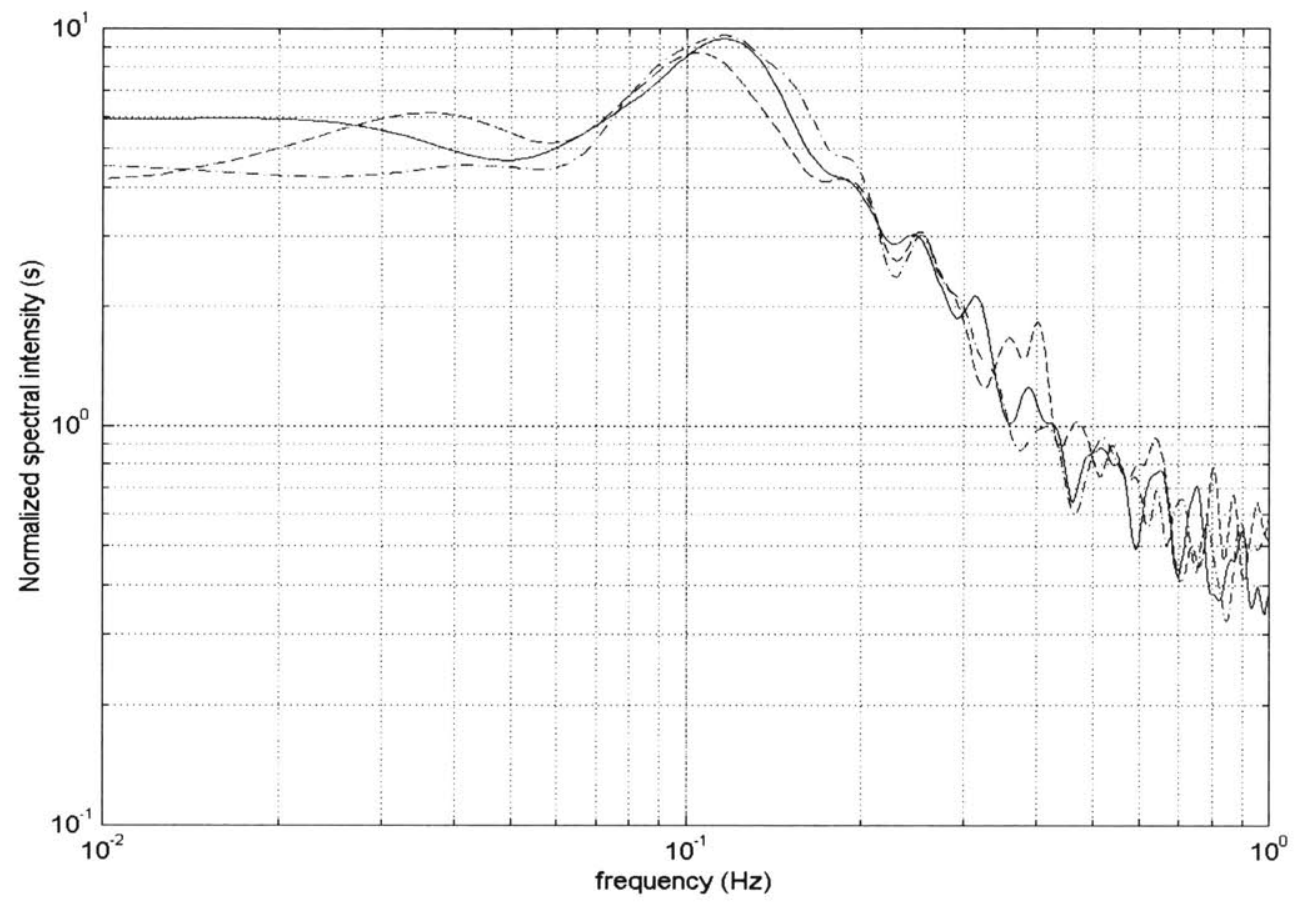

Figure 3.8. Pronounced peaks in the spectra of streamwise velocity in and above the model canopy.

(i) Scenario A $\left(-z / h_{d}=1.37,--z / h_{d}=0.83,-. z / h_{d}=0.72\right) ; f_{r}=1 H z, w_{w}=60$.

(ii) Scenario $C\left(-z / h_{d}=1.06,--z / h_{d}=0.84,-. z / h_{d}=0.84\right) ; f_{r}=2 H z, w_{w}=80$.

(iii) Scenario $G\left(-z / h_{d}=0.93,--z / h_{d}=1.02,-. z / h_{d}=0.80\right) ; f_{r}=4 H z, w_{w}=240$.

Obviously, these characteristic spectral peaks are indicative of strong, periodic velocity oscillations. The streamwise velocity for Scenario $G$ oscillates with a frequency of approximately $0.11 \mathrm{~Hz}$, demonstrated by the peak in the spectrum in Figure 3.8(iii). A raw time series of longitudinal velocity (along with the 1 second moving average) for Scenario $G$ is shown in Figure 3.9, clearly exhibiting oscillations of period $\sim 9 \mathrm{~s}$. 


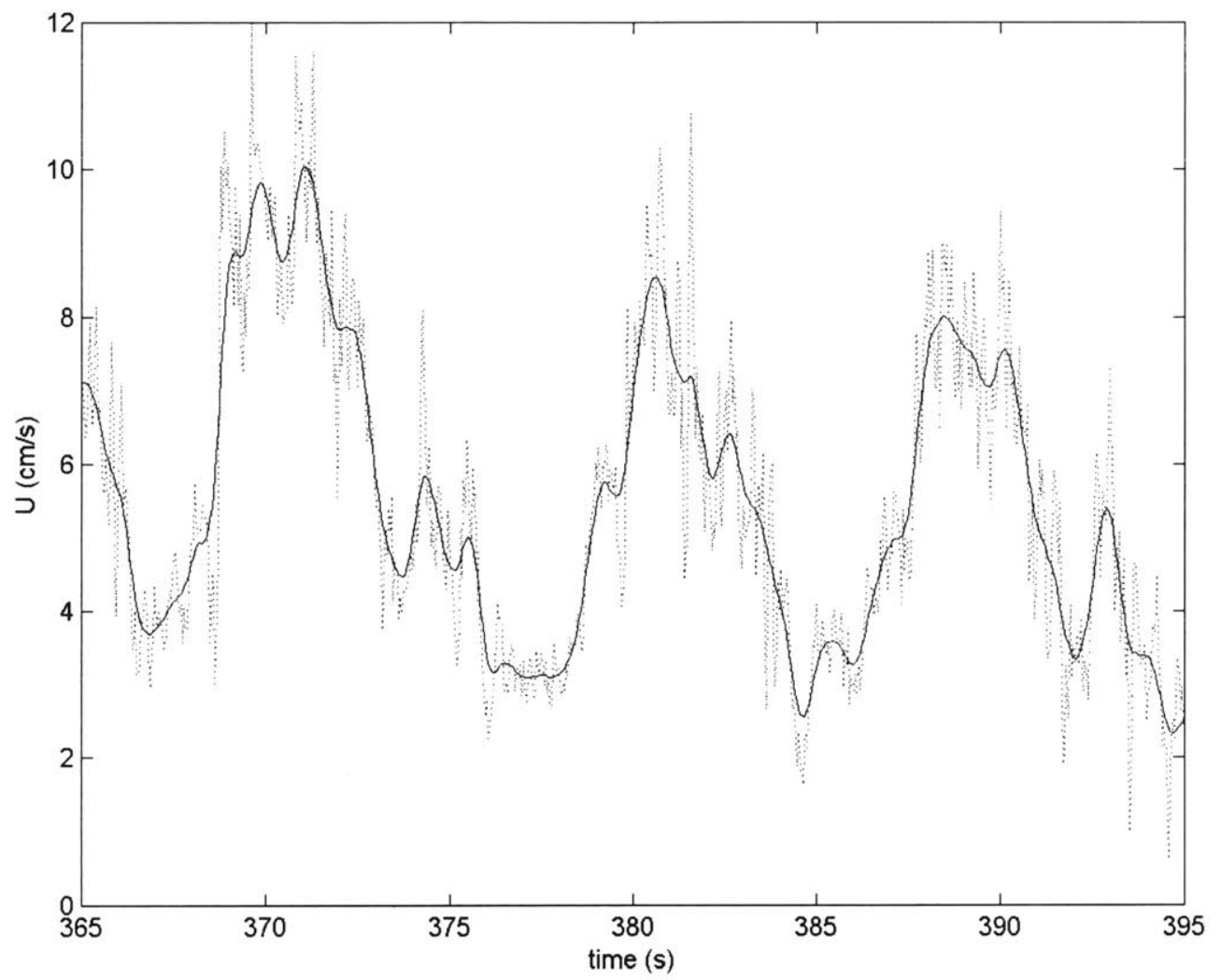

Figure 3.9. Time series of a strongly oscillatory streamwise velocity record, taken at $z / h_{d}=$ 0.93 for Scenario $G$.

: raw time series of longitudinal velocity

- 1 second moving average of longitudinal velocity

Due to the roller-type nature of the generated vortices, oscillations of an identical frequency were expected to be seen in the vertical velocity. Therefore, spectra of vertical velocity were also analyzed. Characteristic peaks of frequencies identical to those in the spectra of longitudinal velocity were indeed found. The presence of a strong peak at exactly double that frequency was also noted, however, as demonstrated in Figure 3.10. 


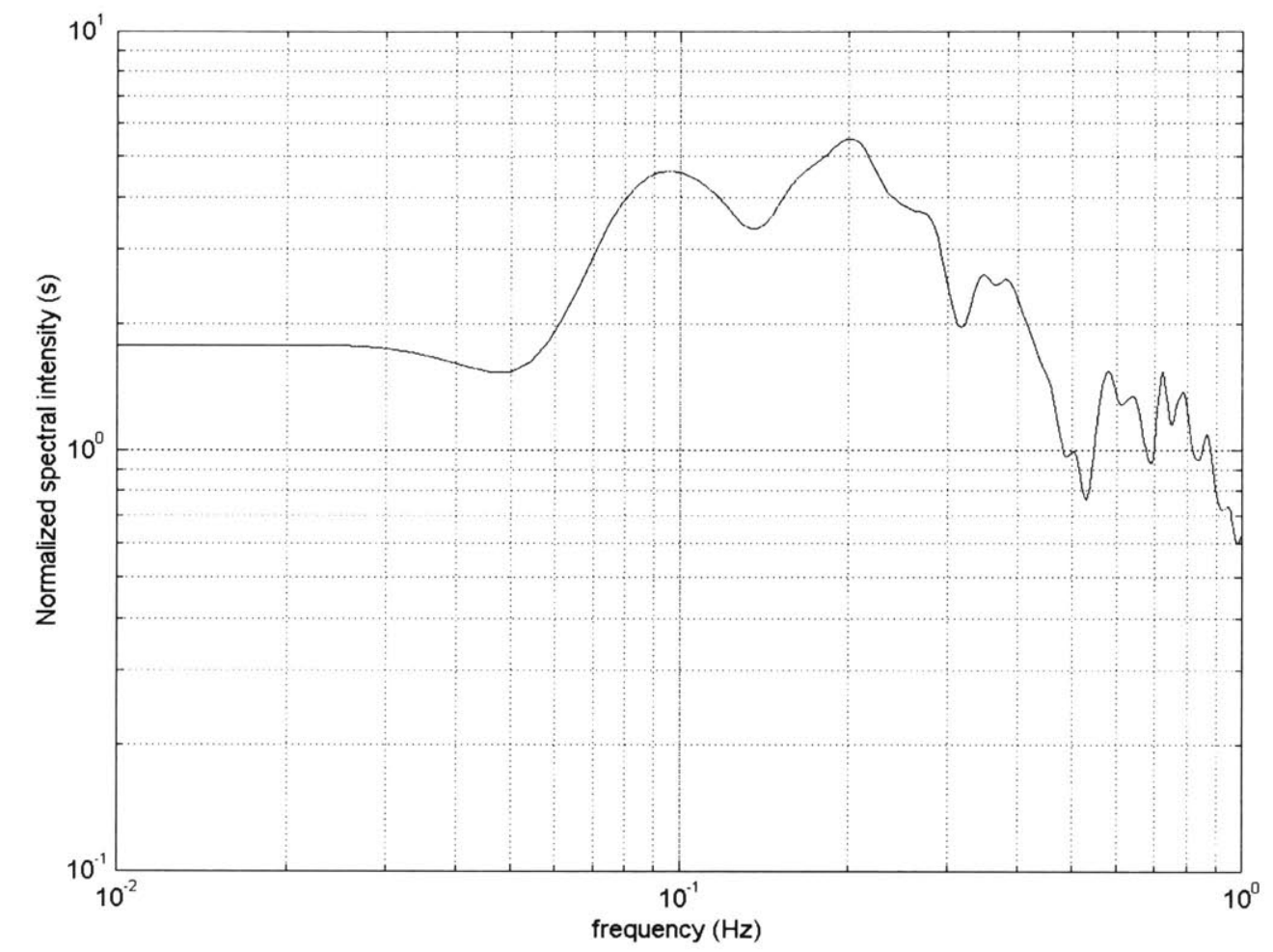

Figure 3.10. Example of the double peak in the power spectrum of vertical velocity. The spectrum is of a time series in Scenario $G\left(z / h_{d}=0.93\right) ; f_{r}=2 H z, w_{w}=80$.

The presence of this harmonic is inconsistent with the roller conceptualization of these vortices, but appears to be related to the vertical velocities generated in between vortices, as noted by Ikeda and Kanazawa (1996). The authors found strong updraft velocities in front of the vortices generated above their flexible, submerged vegetation and there is evidence of mild downward velocities behind the vortices (Figure 3.11). Thus, at any given point, an oscillation of vertical velocity at double the frequency of vortex generation may be observed, as demonstrated by Figure 3.10. 

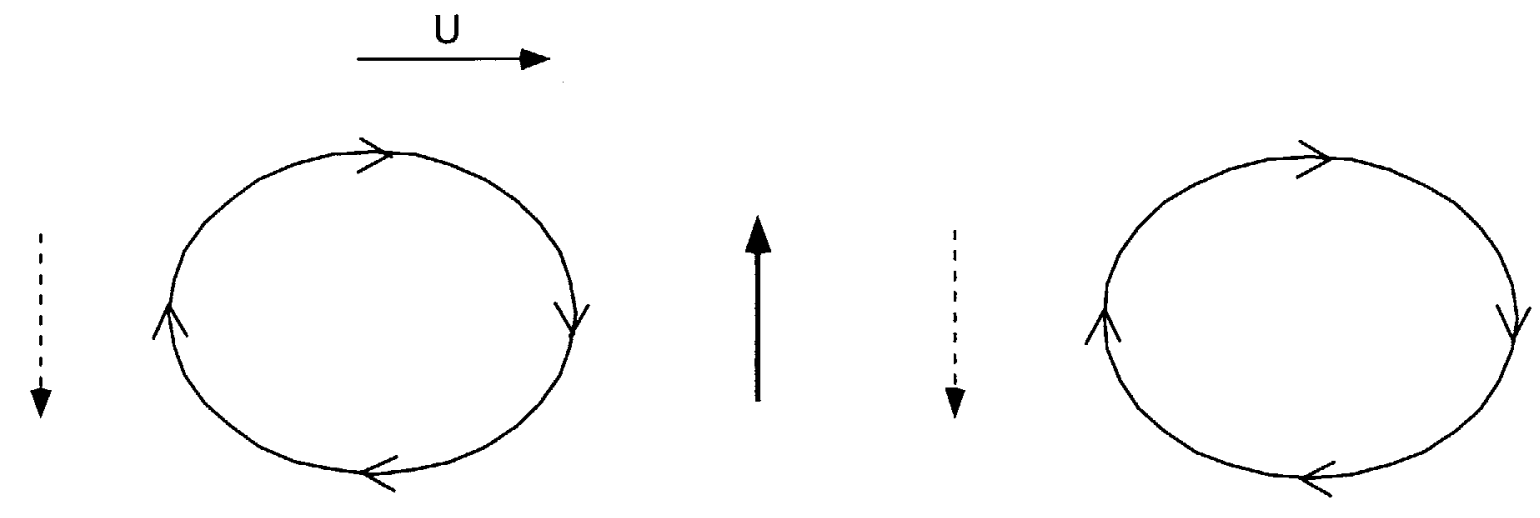

Figure 3.11. Schematic diagram of the sequence of vertical velocities in the vortex street in vegetated flow, as observed by Ikeda and Kanazawa (1996).

The mean monami frequency was obtained from the video footage obtained during the experiments, as discussed in Section 2.3. Over a period of between two and three minutes, each 'monami channel' was observed and the number of waving cycles exhibited by the plants in that channel were counted. The average frequency of the monami, with associated uncertainty (the standard deviation of the three estimates), is shown in Table 3.3 , along with the peak frequencies in the spectra of both longitudinal and vertical velocity and the predicted instability frequency of the generated mixing layer (from Equation 1.2). Note that all velocity records were taken within the central monami channel. 


\begin{tabular}{|c|c|c|c|c|}
\hline Scenario & $\begin{array}{l}\text { Mean monami } \\
\text { frequency }(\mathrm{Hz}) \\
\text { (visual estimate) }\end{array}$ & $\begin{array}{l}\text { Peak in streamwise } \\
\text { velocity spectrum } \\
(\mathrm{Hz})\end{array}$ & $\begin{array}{l}\text { Peaks in vertical } \\
\text { velocity spectrum } \\
(\mathrm{Hz})\end{array}$ & $\begin{array}{l}\text { Predicted mixing layer } \\
\text { instability frequency } \\
(\mathrm{Hz})\end{array}$ \\
\hline $\bar{A}$ & $0.055 \pm 0.008$ & $0.052 \pm 0.006$ & $\begin{array}{l}0.055 \pm 0.005 \\
0.112 \pm 0.005\end{array}$ & $0.051 \pm 0.002$ \\
\hline $\bar{B}$ & $0.028 \pm 0.001$ & $0.028 \pm 0.003$ & $\begin{array}{l}0.030 \pm 0.002 \\
0.057 \pm 0.004\end{array}$ & $0.028 \pm 0.001$ \\
\hline $\bar{C}$ & - & $0.012 \pm 0.001$ & $\begin{array}{l}0.012 \pm 0.001 \\
0.024 \pm 0.002\end{array}$ & $0.010 \pm 0.001$ \\
\hline $\mathrm{D}$ & $0.064 \pm 0.003$ & $0.063 \pm 0.007$ & $\begin{array}{l}0.066 \pm 0.006 \\
0.135 \pm 0.009\end{array}$ & $0.063 \pm 0.002$ \\
\hline $\bar{E}$ & $0.039 \pm 0.005$ & $0.039 \pm 0.006$ & $\begin{array}{l}0.038 \pm 0.003 \\
0.075 \pm 0.005\end{array}$ & $0.037 \pm 0.002$ \\
\hline $\bar{F}$ & - & $0.014 \pm 0.002$ & $\begin{array}{l}0.014 \pm 0.001 \\
0.028 \pm 0.002\end{array}$ & $0.012 \pm 0.001$ \\
\hline $\bar{G}$ & $0.110 \pm 0.007$ & $0.109 \pm 0.012$ & $\begin{array}{l}0.105 \pm 0.004 \\
0.206 \pm 0.010\end{array}$ & $0.111 \pm 0.003$ \\
\hline $\mathrm{H}$ & - & $0.062 \pm 0.006$ & $\begin{array}{l}0.064 \pm 0.004 \\
0.126 \pm 0.003\end{array}$ & $0.060 \pm 0.003$ \\
\hline $\bar{I}$ & - & $0.113 \pm 0.007$ & $\begin{array}{l}0.116 \pm 0.004 \\
0.233 \pm 0.004\end{array}$ & $0.119 \pm 0.007$ \\
\hline
\end{tabular}

Table 3.3. Comparison of observed monami frequencies, predicted mixing layer instability frequency and the frequency of peaks in velocity spectra.

The strong agreement between the mean monami frequency, the predicted instability frequency of the mixing layer and the frequency of oscillations in streamwise velocity confirms the proposal of Ikeda and Kanazawa (1996), namely that all three are related occurrences. The mixing layer profile that develops leads to the generation of vortices that propagate 
downstream. The stream of vortices are responsible for a strongly oscillating streamwise velocity, that causes the progressive, coherent waving of the vegetation.

It is important to note that the lack of waving in the canopy does not imply a lack of coherent structures in the mixing layer. The kinematics of the vortices in such situations (Scenarios C,F,H and I) are insufficient to cause visible deflections of the plants; this is discussed further in Section 3.5.1.

In summary, the data have shown that the monami is generated as follows: the turbulent boundary layer flow encounters the canopy drag, resulting in the redirection of flow over the top of the canopy and the deceleration of lower-lying fluid. This leads to the development of a shear-layer profile, complete with an inflection point. Accordingly, the Kelvin-Helmholtz instability develops and rolls over, creating three-dimensional vortices. In the case of our experimental model, this all appears to occur within the first $0.7 \mathrm{~m}$ of the canopy. These vortices are maintained by the velocity shear across the top of the canopy and progress downstream. Therefore, each plant observes a stream of vortices and, resultingly, a oscillating streamwise velocity. This oscillating velocity causes the coherent and progressive waving of the vegetation.

It is interesting to note that the monami is visible as the propagation of forward plant deflection along the canopy, indicating the progression of high streamwise velocity, as represented in Figure 3.12. 


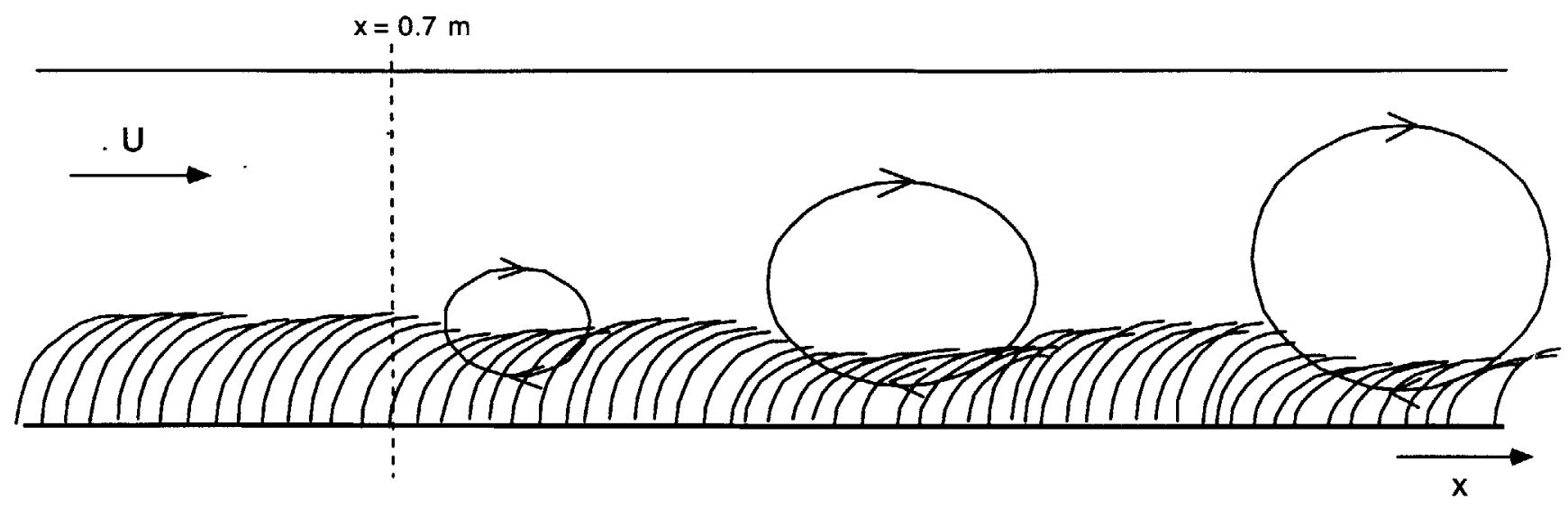

Figure 3.12. Schematic diagram of plant response to the passage of the street of elliptical vortices in the generated mixing layer. The monami is observed as a progression of forward plant deflection.

This is counterintuitive, as the shear of the mean velocity profile would create a vortex rotating clockwise in Figure 1.1. Therefore, the canopy would encounter lower velocities than usual as a vortex passed, given that the canopy lies below the axis of vortex propagation. This is commonly seen in pure mixing layers, where the vortex circulation can be so pronounced that instantaneous reversal of the velocity can be seen as a vortex passes (Dimotakis et al., 1981). Seemingly, this is not the case here and prompted the following study of the speed of propagation of these coherent structures.

\subsection{Vortex velocities}

The second set of measurements in these experiments were taken to estimate the longitudinal velocity of the coherent vortices $\left(U_{v}\right)$ generated in the mixing layer. This involved the use of two ADV probes, as shown in Figure 3.13. The idea behind the measurement of vortex velocity was that since individual vortices are maintained by the shear of the mixing 
layer, the change in velocity as an individual vortex passes can be measured at more than one point along the canopy.

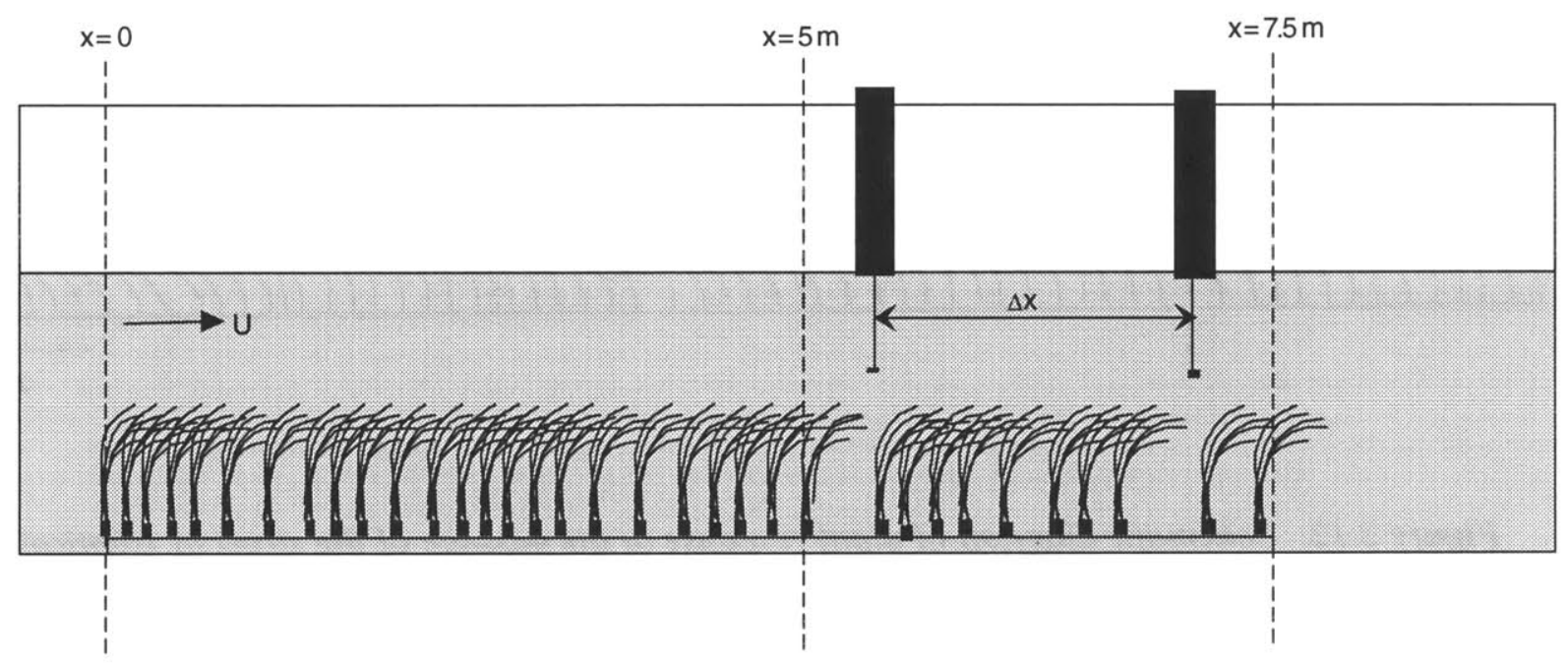

Figure 3.13. Experimental configuration for the measurement of the speed of progression of the coherent vortices.

The two probes were separated by a distance ranging between $0.5 \mathrm{~m}$ and $1.6 \mathrm{~m}$, depending upon the mean velocity of the mixing layer region $(\bar{U})$. The separation distance was chosen such that the travel time of a vortex from the upstream probe to the downstream probe $\left(t_{v}\right)$ was large enough to make negligible any error incurred by the manual commencement of both probe recordings, but small enough so that the characteristics of the vortex would not change appreciably as it progressed along the canopy. The probes spanned a length of canopy in which the mean velocity profile, and hence the momentum thickness, changed little. With reference to Figure 3.13, this meant that the upstream probe was never placed less than $5.0 \mathrm{~m}$ along the canopy. For each flow scenario, three vortex velocity estimates were obtained from ten minute velocity records obtained simultaneously by the ADV probes. With both probes at the same lateral position across the flume, estimates were obtained at $y=0, y=+3 \mathrm{~cm}$ and 
$y=-3 \mathrm{~cm}$ for all flow scenarios. Since all these positions lay in the same 'vortex channel', this was done more for repetition than to capture lateral variability.

This second set of measurements was performed after the completion of the first set. Consequently, the valve had to be carefully adjusted to recreate the same flowrate that was used initially. However, due to inaccuracies that might be incurred (especially at low flowrates where the error in the estimation of the flow rate is comparatively high), the mean velocity of the mixing layer $(\bar{U})$ had to be re-estimated for all flow scenarios. Therefore, all measurements were taken at $\bar{z}$, the mean height of the mixing layer for each individual scenario (as shown in Table 3.2). The average of the three mean values of longitudinal velocity was designated as the new value of $\bar{U}$. For the scenarios with a low flowrate $(<20 \mathrm{gpm})$, the disagreement between the two values was significant, ranging between $1 \%$ and $27 \%$; for higher flowrates, the new value of $\bar{U}$ always agreed with the old value to within $10 \%$.

To obtain an estimate of vortex velocity, the crosscorrelation between the two simultaneous velocity records was examined. The longitudinal velocity record of the downstream probe was successively lagged against the same record of the upstream probe, until the crosscorrelation between the two records $\left(r_{k}\right)$ was maximized.

$$
r_{k}=\frac{1 / N \sum_{n=1}^{N-k}\left(u_{1, n}-U_{1}\right)\left(u_{2, n+k}-U_{2}\right)}{u_{r m, 1} \cdot u_{r m s, 2}}
$$

where the subscripts 1 and 2 refer to the upstream and downstream velocity records respectively, $k$ is the lag between the two records, $n$ is the number of the data point in the velocity record and $N$ is the total number of points in the velocity record. The crosscorrelation reflects the correlation between the two velocity records when the downstream record is shifted forward by $k$ data points. It is expected that the crosscorrelation between the two records would be maximized when the lag is equal to the time taken for an individual vortex to travel from the 
upstream probe to the downstream probe $\left(t_{\text {raw }}\right)$. Often, several peaks were present in the crosscorrelation function, indicating that the separation distance of the probes was large enough such that more than one vortex was present between them at any given time. However, there was always one clearly dominant peak, invariably at a time lag that corresponded to a vortex velocity in the vicinity of $\bar{U}$. The lag associated with this peak was assumed to be the time taken for a vortex to travel between the ADV probes. The MATLAB code used to compute the cross-correlation functions is shown in Appendix C. Sample plots of the cross-correlation between the two velocity records as a function of lag are shown in Figure 3.14.

(a)

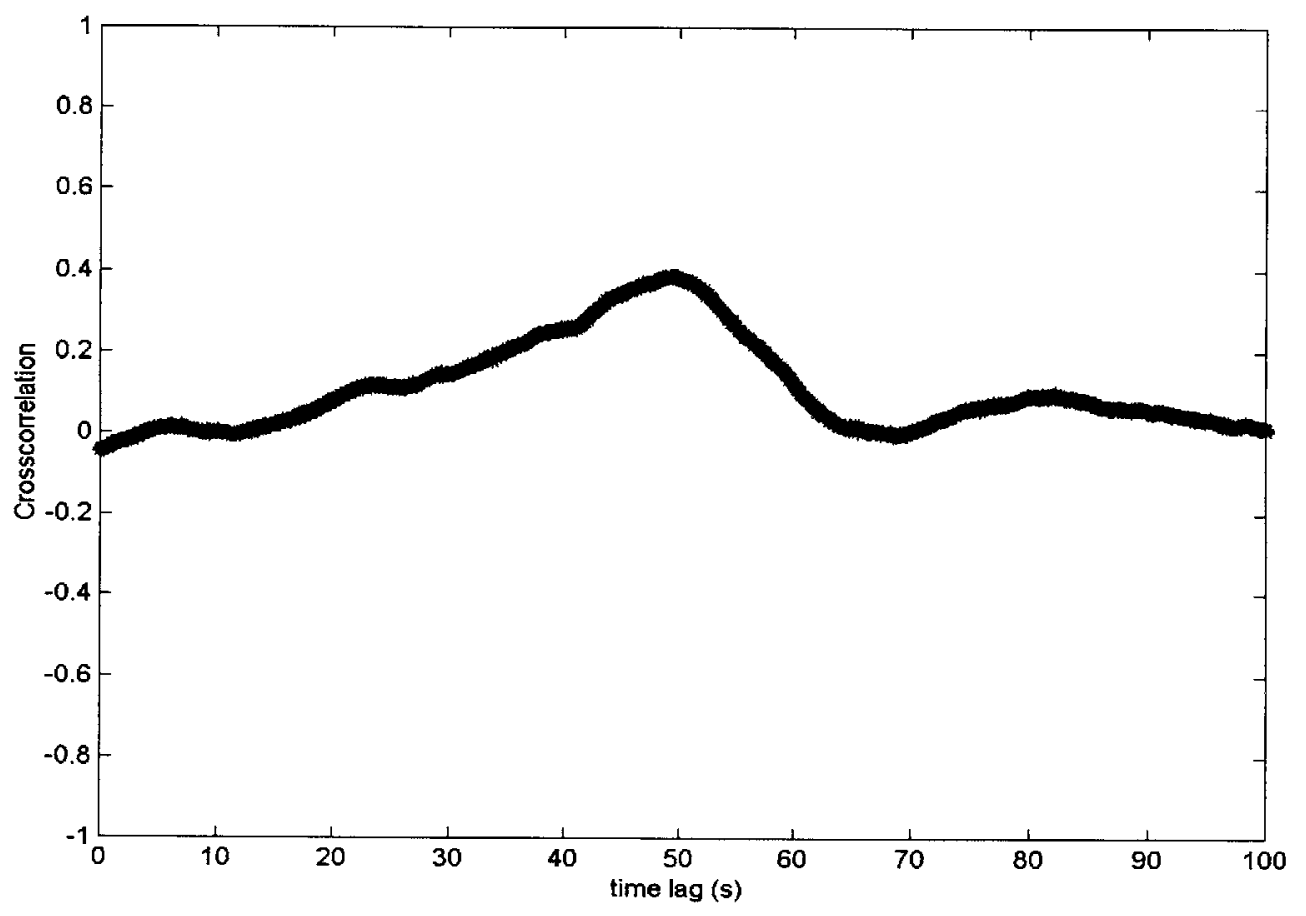


(b)

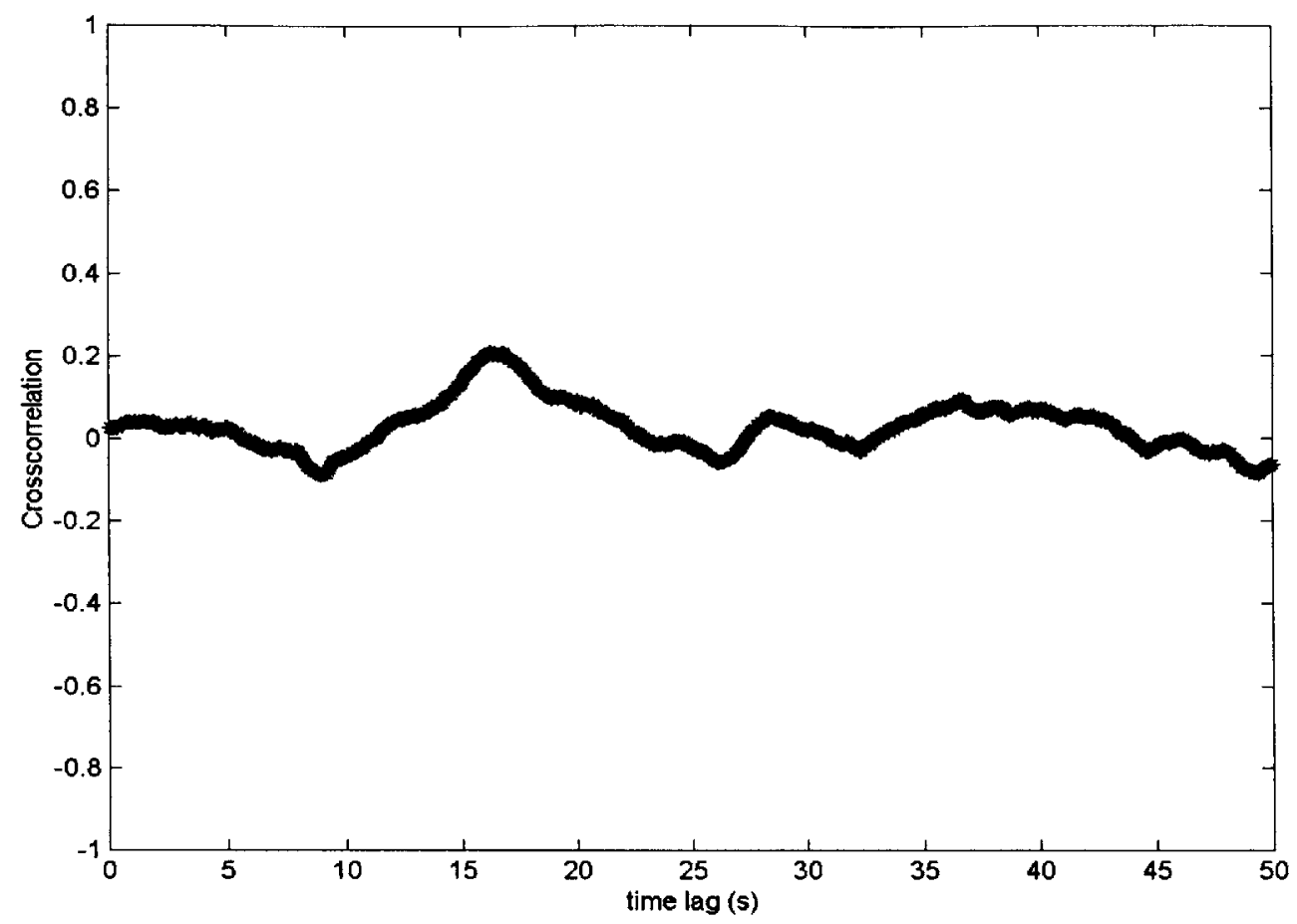

Figure 3.14. Examples of the cross-correlation between two lagged, simultaneous velocity records taken at different points along the canopy.

(a) Scenario $F, y=-3 \mathrm{~cm}, \Delta x=0.62 \mathrm{~m}$

(b) Scenario $G, y=+3 \mathrm{~cm}, \Delta x=1.55 \mathrm{~m}$

As a form of quality control, measurements that produced a maximum crosscorrelation of less than 0.08 (a nominal value) were rejected and the corresponding experiment was repeated. The vortex velocity was calculated using the estimated travel time of the vortex:

$$
U_{v}=\frac{\Delta x}{t_{v}}
$$

The resulting estimates of vortex velocity are shown in Table 3.4. Note that the uncertainty in the vortex velocity is much greater than any uncertainty in the re-estimation of $\bar{U}$; the latter 
has thus been neglected.

\begin{tabular}{|c|c|c|c|c|c|c|c|}
\hline Scenario & $y(\mathrm{~cm})$ & $\Delta x(\mathrm{~cm})$ & $t_{v}(\mathrm{~s})$ & $U_{v}\left(\mathrm{cms}^{-1}\right)$ & $\bar{U}\left(\mathrm{cms}^{-1}\right)$ & $\frac{U_{v}}{\bar{U}}$ & $\frac{U_{v}}{U_{2}}$ \\
\hline $\mathrm{A}$ & $\begin{array}{r}0 \\
-3 \\
+3\end{array}$ & $\begin{array}{l}161.1 \\
154.6 \\
168.2\end{array}$ & $\begin{array}{l}14.81 \\
15.17 \\
16.87\end{array}$ & $\begin{array}{c}10.88 \\
10.19 \\
9.97\end{array}$ & 7.12 & $1.45 \pm 0.07$ & $0.84 \pm 0.04$ \\
\hline B & $\begin{array}{r}0 \\
-3 \\
+3\end{array}$ & $\begin{array}{l}154.6 \\
153.6 \\
154.4\end{array}$ & $\begin{array}{l}43.86 \\
43.21 \\
43.41\end{array}$ & $\begin{array}{l}3.52 \\
3.55 \\
3.56\end{array}$ & 2.79 & $1.27 \pm 0.01$ & $0.63 \pm 0.01$ \\
\hline $\mathrm{C}$ & $\begin{array}{r}0 \\
-3 \\
+3\end{array}$ & $\begin{array}{l}62.4 \\
52.7 \\
63.4\end{array}$ & $\begin{array}{l}50.23 \\
44.95 \\
54.43\end{array}$ & $\begin{array}{l}1.24 \\
1.17 \\
1.16\end{array}$ & 0.973 & $1.22 \pm 0.04$ & $0.69 \pm 0.02$ \\
\hline D & $\begin{array}{r}0 \\
-3 \\
+3\end{array}$ & $\begin{array}{l}153.6 \\
157.5 \\
154.3\end{array}$ & $\begin{array}{l}14.20 \\
14.04 \\
14.24\end{array}$ & $\begin{array}{l}10.82 \\
11.22 \\
10.84\end{array}$ & 7.45 & $1.47 \pm 0.03$ & $0.88 \pm 0.02$ \\
\hline $\mathrm{E}$ & $\begin{array}{r}0 \\
-3 \\
+3\end{array}$ & $\begin{array}{l}151.6 \\
153.7 \\
152.8\end{array}$ & $\begin{array}{l}35.83 \\
35.83 \\
37.24\end{array}$ & $\begin{array}{l}4.23 \\
4.29 \\
4.10\end{array}$ & 3.03 & $1.25 \pm 0.03$ & $0.72 \pm 0.02$ \\
\hline $\mathrm{F}$ & $\begin{array}{r}0 \\
-3 \\
+3\end{array}$ & $\begin{array}{l}62.0 \\
61.5 \\
62.4\end{array}$ & $\begin{array}{l}47.97 \\
49.22 \\
46.68\end{array}$ & $\begin{array}{l}1.29 \\
1.25 \\
1.34\end{array}$ & 1.12 & $1.16 \pm 0.03$ & $0.79 \pm 0.02$ \\
\hline $\mathrm{G}$ & $\begin{array}{c}0 \\
0 * \\
-3 \\
+3\end{array}$ & $\begin{array}{l}156.6 \\
155.6 \\
156.9 \\
154.6\end{array}$ & $\begin{array}{l}18.00 \\
17.35 \\
16.62 \\
16.70\end{array}$ & $\begin{array}{l}8.70 \\
8.97 \\
9.44 \\
9.26\end{array}$ & 7.12 & $1.28 \pm 0.05$ & $0.84 \pm 0.03$ \\
\hline $\mathrm{H}$ & $\begin{array}{c}0 \\
-3 \\
+3\end{array}$ & $\begin{array}{l}88.0 \\
88.0 \\
88.0\end{array}$ & $\begin{array}{l}27.44 \\
27.94 \\
27.89\end{array}$ & $\begin{array}{l}3.21 \\
3.15 \\
3.16\end{array}$ & 2.69 & $1.18 \pm 0.01$ & $0.77 \pm 0.01$ \\
\hline I & $\begin{array}{r}0 \\
-3 \\
+3\end{array}$ & $\begin{array}{l}88.0 \\
88.0 \\
88.4\end{array}$ & $\begin{array}{l}19.67 \\
20.17 \\
20.09\end{array}$ & $\begin{array}{l}4.47 \\
4.36 \\
4.40\end{array}$ & 4.03 & $1.09 \pm 0.01$ & $0.84 \pm 0.01$ \\
\hline
\end{tabular}

Table 3.4. Estimation of vortex velocity based on crosscorrelation of two velocity records at different longitudinal locations.

* extra measurement taken due to high variability in the data 


\subsubsection{Effects of the rapid convective velocity of the vortices}

It is interesting to note that the ratio of vortex velocity to mean mixing layer velocity is always greater than unity. This agrees with the flow visualization findings of Ikeda and Kanazawa (1996) but contrasts measurements in pure mixing layers (i.e. those without the vegetation drag) where the ratio is almost exactly unity (Dimotakis et al., 1981; Ho and Huerre, 1984; Panides and Chevray, 1990). There exists a clear relationship between the relative vortex velocity and the mixing layer thickness, as demonstrated in Figure 3.15. The intercept of the curve is, interestingly enough, at a value close to unity. This suggests that in the initial stages of mixing layer development, vortex velocities are similar to those in a pure mixing layer. As the thickness of the layer grows, however, the vortices become disproportionately affected by the high-stream velocities of the mixing layer, leading to increased convective velocities of the coherent structures. This is highlighted by the fact that as the mixing layer thickness (and hence the vortex size) grows, so too does the vortex velocity relative to $\bar{U}$. In other words, as the vortex is able to encounter more of the high-stream velocities, it travels faster as a result. As expected, the vortex velocity never exceeds the maximum velocity of the mixing layer, $U_{2}$ (Table 3.4). 


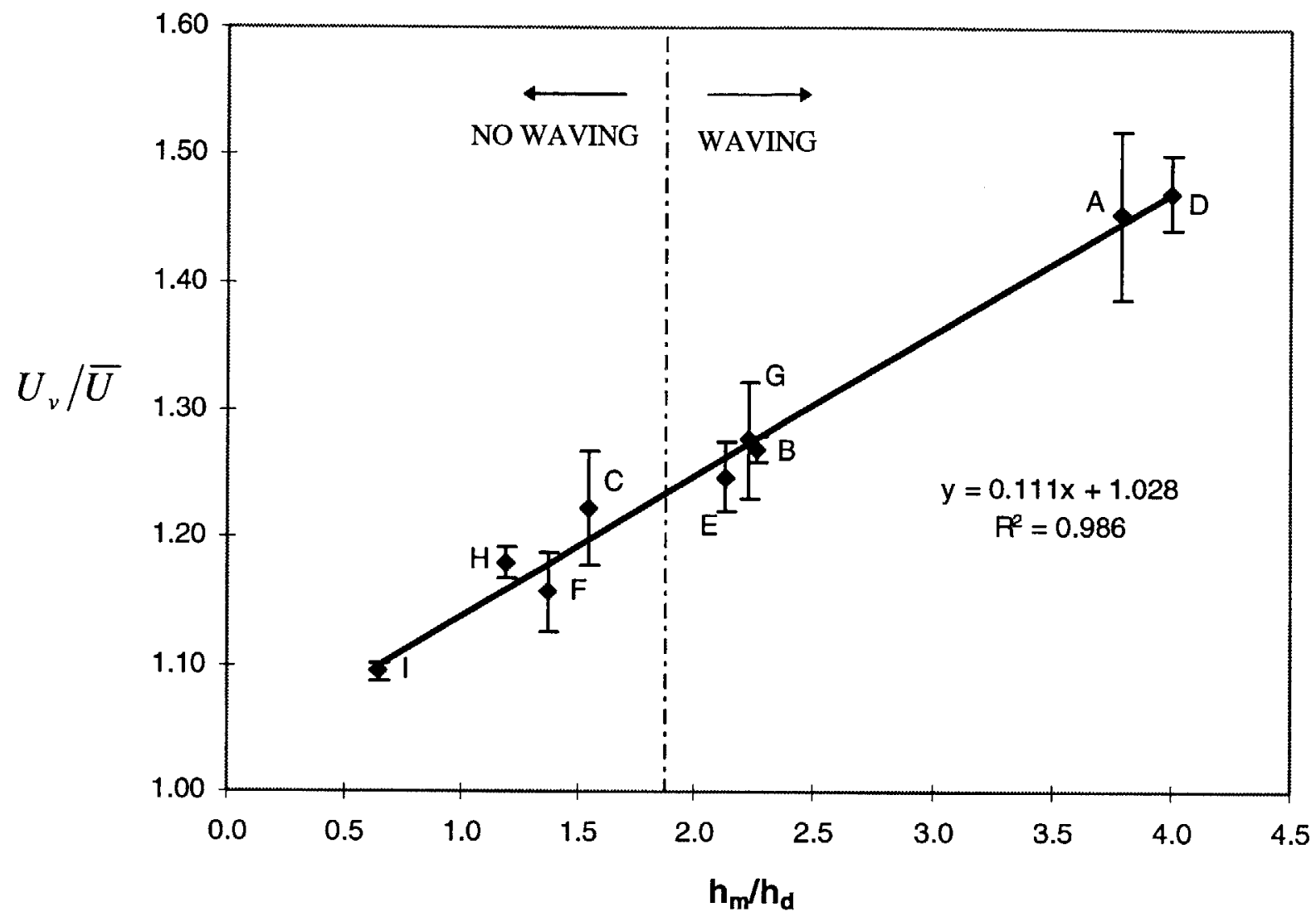

Figure 3.15. Evidence of the linear relationship between the ratio of vortex velocity to mean mixing layer velocity with the non-dimensionalized mixing layer height. Error bars indicate one standard deviation from the lateral average. Data point labels indicate the flow scenario in which the measurement was taken.

The observed vortex velocities, while in striking contrast to that expected based on mixing layer theory, are in agreement with the flow visualization estimates of Ikeda and Kanazawa (1996). In terrestrial canopies, an accepted value of $U_{\nu}$ is $1.8 U_{h}$, according to Finnigan (1979a), where $U_{h}$ is the mean velocity at the top of the canopy. In this study, the average $\bar{U} / U_{h}$ ratio was 1.22 . Assuming a similar ratio for terrestrial canopies, where the center of the mixing layer is consistently above the top of the canopy (Raupach et al., 1996), the relative vortex velocity $\left(U_{v} / \bar{U}\right)$ is approximately 1.5 . This is consistent with our findings, 
where in the cases most resembling the unbounded terrestrial flows (i.e. Scenarios A and D), we see a very similar ratio. Although not suggested by the linear relationship in Figure 3.15 (which spans a narrow range of $h_{m} / h_{d}$ ), it is expected that as an unconfined canopy condition is approached, the value of $U_{v} / \bar{U}$ asymptotes to a value of approximately 1.5 .

The observation of the monami as a downstream progression of a localized region of forward plant deflection indicates that the speed of vortex propagation is sufficiently high, and their circulation sufficiently slow, that as a vortex passes, the streamwise velocity at the top of the canopy increases. This is easier to comprehend when it is considered that the top of the canopy lies up to $1.5 \theta$ below the axis of vortex propagation, and experiences a much lower mean velocity than the center of the mixing layer. Given that the vortices are also travelling up to $50 \%$ faster than the mean mixing layer velocity, the advection speed of these vortices, relative to the mean velocity at the top of the canopy, is so large that its passage is accompanied by a velocity increase, despite its circulation.

Given the data presented in Figure 3.15, the curve detailing the conditions required for observation of the monami (Figure 3.4) can now be interpreted in more detail. For very large water depths, the mixing layer thickness is not restricted by the free surface, allowing $h_{m}$ to grow large, leading to a high vortex velocity. Consequently, a monami is observed for virtually all flow rates, except when the mean velocity is so low that even the (comparatively) rapidly propagating vortices are not strong enough to cause a deflection of the eelgrass blades. As the free surface is lowered, the mixing layer thickness becomes restricted by the upper boundary. As a result, the relative vortex velocity $\left(U_{v} / \bar{U}\right)$ decreases as the water depth becomes shallower (Figure 3.15). Consequently, greater velocities are needed to bend the plants over sufficiently for the $h_{m} / h_{d}$ ratio to grow large enough to result in sufficiently rapid vortex propagation. 


\subsubsection{Critical parameter for monami observation}

Given that the flexibility of submerged aquatic vegetation varies widely, it seems reasonable to attempt to develop a general criterion for the presence of the monami in submerged aquatic vegetation. The lower portion of the curve in Figure 3.4 (i.e. that with an increasing trigger interface velocity) corresponds to a maintenance of an $H / h_{d}$ value $\left[\approx h_{m} / h_{d}\right.$ for such shallow depths] of 1.7. That is, the velocity must increase sufficiently to deflect the plants such that $H / h_{d}>1.7$ before the monami can occur. Importantly, this ties in perfectly with Figure 3.15, where the criterion for the observation of waving is $h_{m} / h_{d}>1.6-2.0$. Additionally, this compares favorably with the work of Vivoni (1998) who employed relatively shallow water depths such that mixing layer thickness was consistently restricted by the free surface. The monami was not observed until $H / h\left(\approx h_{m} / h_{d}\right)$ reached 1.75 .

Hence, there clearly exist two separate regimes of the mixing layer flow. The first regime involves the shear layer not extending up fully to the free surface and therefore remaining unconstrained by the limited depth. The second involves the shear layer being able to grow no more, restricted by the presence of the boundary. The depth at which the transition occurs depends on the growth rate of the mixing layer and the longitudinal distance into the canopy. Although the qualitative nature of the curve in Figure 3.4 will be invariant, the magnitudes of the trigger velocities will obviously be a function of plant flexibility (stiffer plants will require a much greater current to exhibit the monami). 


\subsubsection{Momentum transport by the coherent structures}

The correlation coefficient $r_{u w}$ is an important turbulent parameter in this study as it is an indication of the efficiency of the turbulent vertical transport of streamwise momentum. It is defined statistically as the correlation coefficient between the longitudinal and vertical turbulent fluctuations:

$$
r_{u w}=\frac{\overline{u^{\prime} w^{\prime}}}{u_{r m s} \cdot w_{r m s}}
$$

This parameter can be used to infer size, location and strength characteristics of the generated vortices. It is worth noting that in open-channel flow, the correlation coefficient reaches a maximum (in magnitude) of approximately -0.32 . However, in flows through terrestrial vegetation, coefficients of up to -0.5 are observed (Raupach et al., 1996), indicative of the importance of the coherent structures in momentum transport and by implication, scalar fluxes. The negative signs of the correlation coefficients in these flows result from the predominance of ejection and sweep events, causing a downward turbulent transfer of momentum.

Figures 3.16 and 3.17 show the vertical profiles of the correlation coefficient for the flow scenarios with and without the monami respectively. Note that in these figures, $h_{p}$, the depth of penetration of Reynolds stress into the canopy, is shown. This quantity is defined as the vertical distance into the canopy (from above) at which the Reynolds stress, and thus the correlation coefficient, reaches zero. 


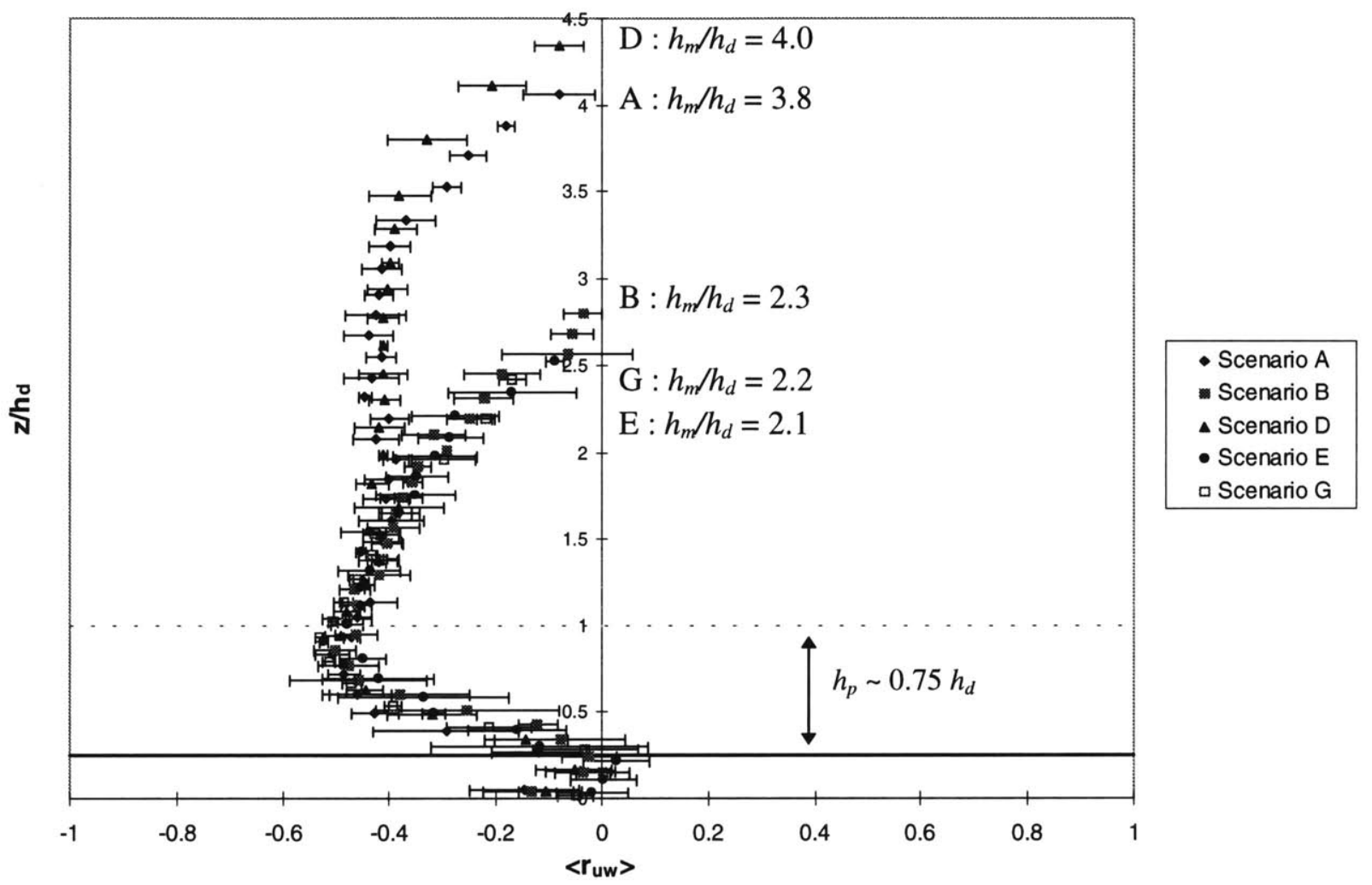

Figure 3.16. Profiles of the correlation coefficient for flow scenarios with the monami. Horizontal bars indicate one standard deviation from the lateral average. The dashed line represents the top of the canopy and the solid line shows the extent of penetration of Reynolds stress into the canopy. Data labels show the $h_{m} / h_{d}$ ratio for each flow scenario. 


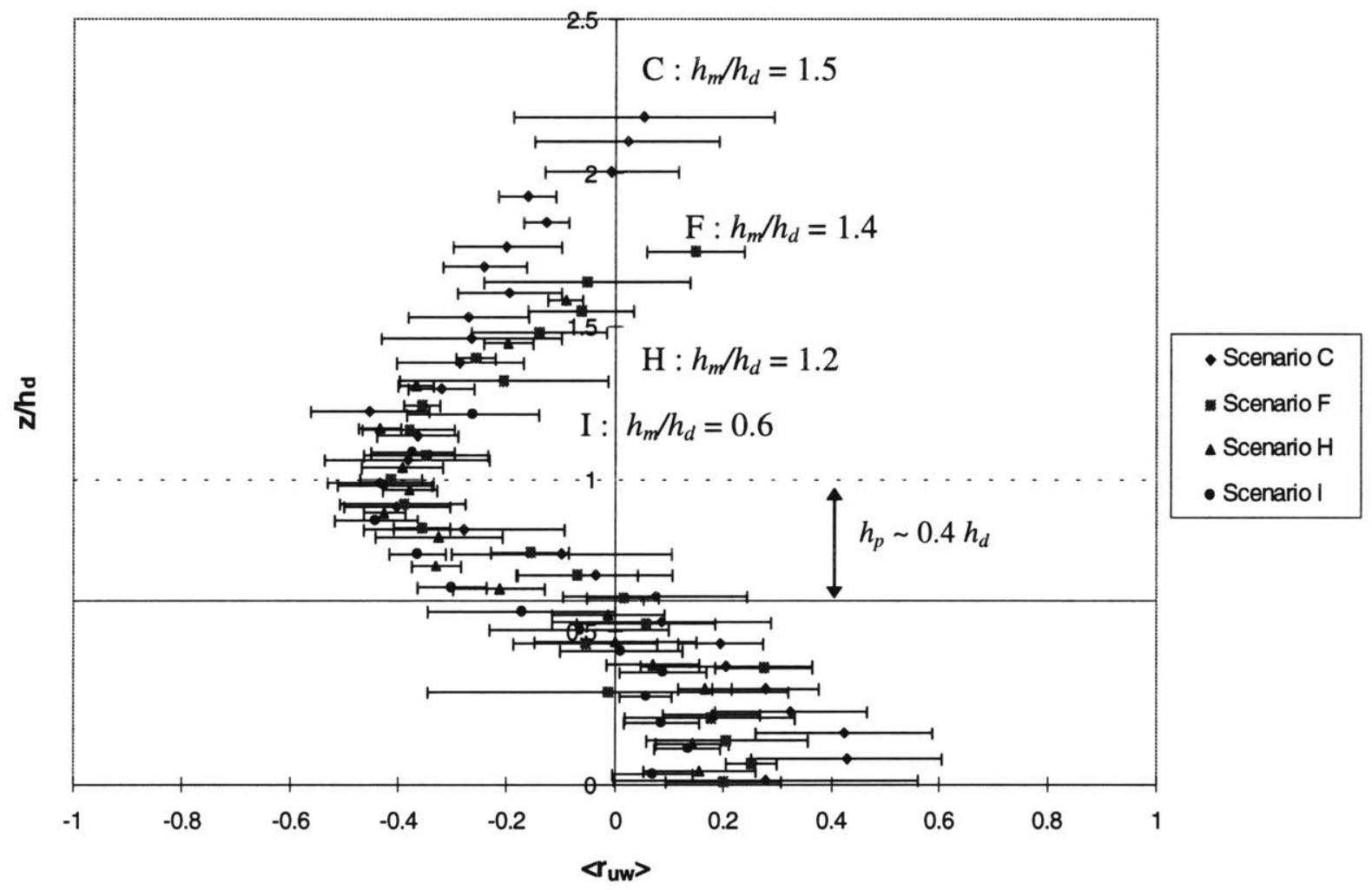

Figure 3.17. Profiles of the correlation coefficient for flow scenarios without the monami. Horizontal bars indicate one standard deviation from the lateral average. The dashed line represents the top of the canopy and the solid line shows the extent of penetration of Reynolds stress into the canopy. Data labels show the $h_{m} / h_{d}$ ratio for each flow scenario.

Regardless of whether the monami is present, $r_{u w}$ reaches a maximum magnitude at the inflection point where the shear is maximized (i.e. just below the top of the canopy). In the case of the waving canopy, the maximum value reached is approximately -0.5 , in accordance with the observations of Raupach et al. (1996) in terrestrial canopies. Above this point, the vertical profile of $r_{u w}$ reflects the kinematic influence of the vortices, the size of which are restricted by the thickness of the mixing layer. Figures 3.16 and 3.17 tie in to Figure 3.15 and demonstrate 
the importance of two vertical length scales: the canopy height and the mixing layer thickness. The comparatively thick mixing layers of Scenarios A and D allow a much larger vortex to develop, leading to a thicker zone of efficient downward transfer of streamwise momentum. In all cases, the efficiency of momentum transfer is maximized at the top of the canopy, and remains considerably larger than that in a turbulent boundary layer within the mixing layer, due to the coherent structures that develop.

As with the vertical profiles of $w_{r m s}$ (Figure 3.7), the profiles of $r_{u w}$ are asymmetric about their maximum value (at $z \sim h_{d}$ in all cases). The decrease of $r_{u w}$ is much more rapid below $z=h_{d}$ than it is above that point, highlighting the inability of the vortices to penetrate into the canopy., due to the drag exerted by the vegetation. Comparison of Figures 3.16 and 3.17 demonstrates that plant waving, however, allows a considerably greater penetration of Reynolds stress (associated with the vortices) into the canopy $\left(h_{p \text {,waving }} \sim 0.75 h_{d} ; h_{p, n o n-w a v i n g}\right.$ $\left.\sim 0.4 h_{d}\right)$. The relative drag force exerted by stationary blades is considerably greater than that exerted by blades that deflect under strong sweep events. Consequently, the vortex structure is broken up by the non-waving blades, leading to a diminished penetration of Reynolds stress. The structure of the profile of $r_{u w}$ above $z=h_{d}$ is similar, regardless of plant waving, with a high value being maintained from the top of the canopy to the top of the generated mixing layer.

It is also noticeable that, especially within the canopy, there is a much greater spatial variability in $r_{u w}$ when the canopy is not waving. This results from the lack of penetration of large, canopy-scale turbulence into the canopy. Thus, the Reynolds stress arises predominantly from stem-scale turbulence, which would otherwise be superceded by the canopy-scale vortical structures of the mixing layer. The spatial heterogeneity created by the distributed wakes therefore creates strong lateral variability in $r_{u w}$. 
The strong positive correlation in the lower halves of the canopies that do not exhibit the monami is symptomatic of a region of 'reverse shear' (i.e. where the velocity increases as the bed is approached). This is explored further in Section 3.5.4.

\subsubsection{Vortex size}

The correlation coefficient profiles within the generated mixing layers are shown in Figures 3.18 and 3.19. These figures show essentially the same data as Figures 3.16 and 3.17, but with a non-dimensionalized vertical axis, in an attempt to collapse the data. Figure 3.16 shows that in a waving canopy, the vortical structure has a vertical extent of approximately $1.5-3.5 h_{d}$, using -0.3 as the approximate (cutoff) value for the far less coherent boundary layer turbulence. For the large-scale turbulence above the flexible canopy of Wallace et al. (1998), the vertical Eulerian length scale was approximately $1.5 h_{d}$. Allowing for the omitted factor of $2 \pi$ in his estimation of integral length scales - the inclusion of which yields length scales corresponding more accurately to actual eddy sizes (Wallace et al., 1998) - Vivoni (1998) found the vertical Eulerian length scale to be of the order of $1.5-2.5 h_{d}$. Ikeda and Kanazawa (1996) observed the size of generated vortices to be approximately $1.5-2 h_{d}$. The relationship between such an integral length scale and the canopy height is, however, secondary and would be expected to vary considerably, especially in the initial stages of the canopy when mixing layer growth is pronounced. The corresponding relationship with a mixing layer parameter is more relevant and is expected to be more consistent between different canopies and flow conditions, as confirmed by Figure 3.18 . 


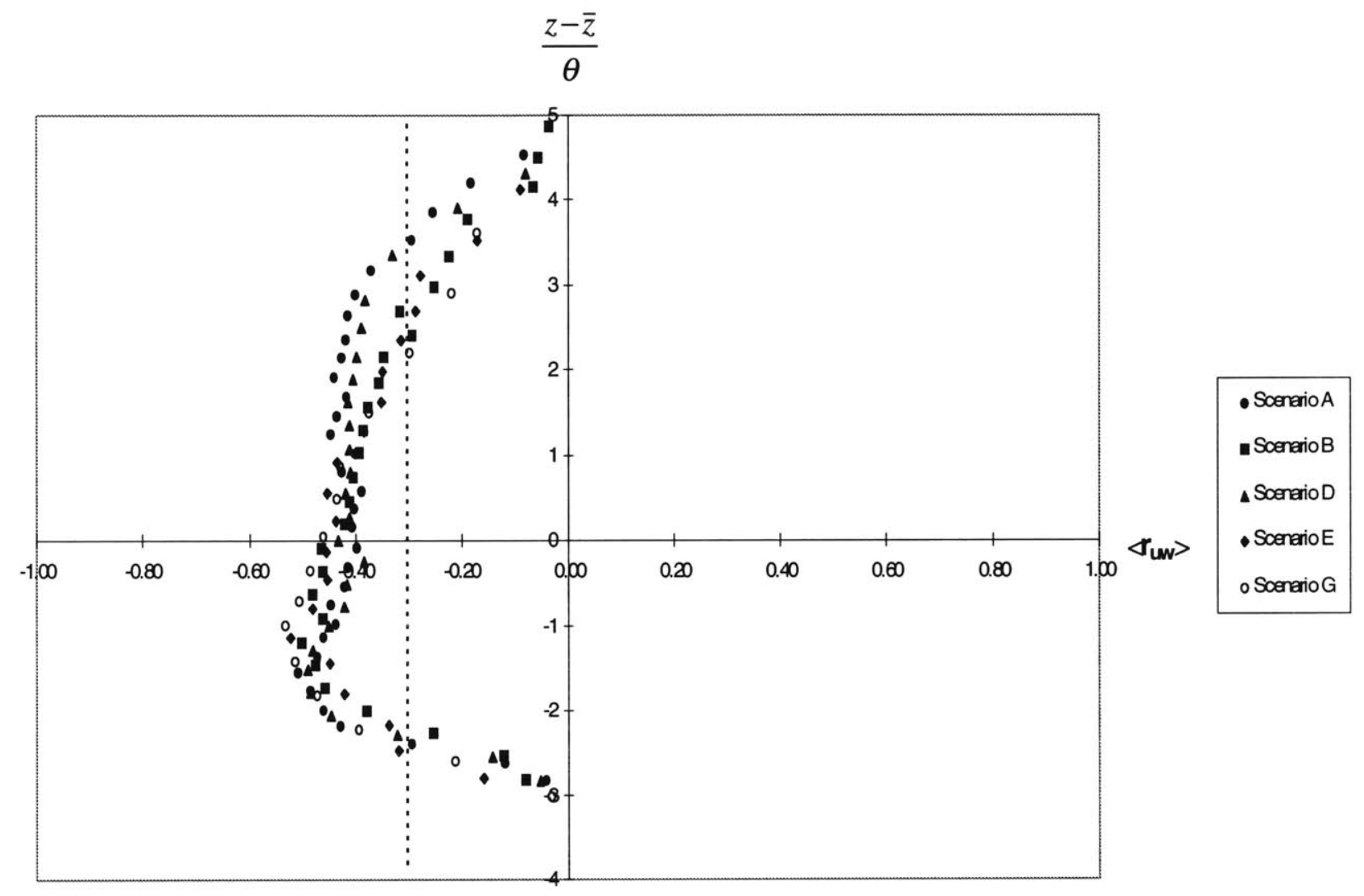

Figure 3.18. Correlation coefficient profiles through the mixing layer for all flow scenarios with the monami. The dashed line indicates the maximum magnitude of $r_{u w}$ observed in boundary layer flow ( -0.3). 


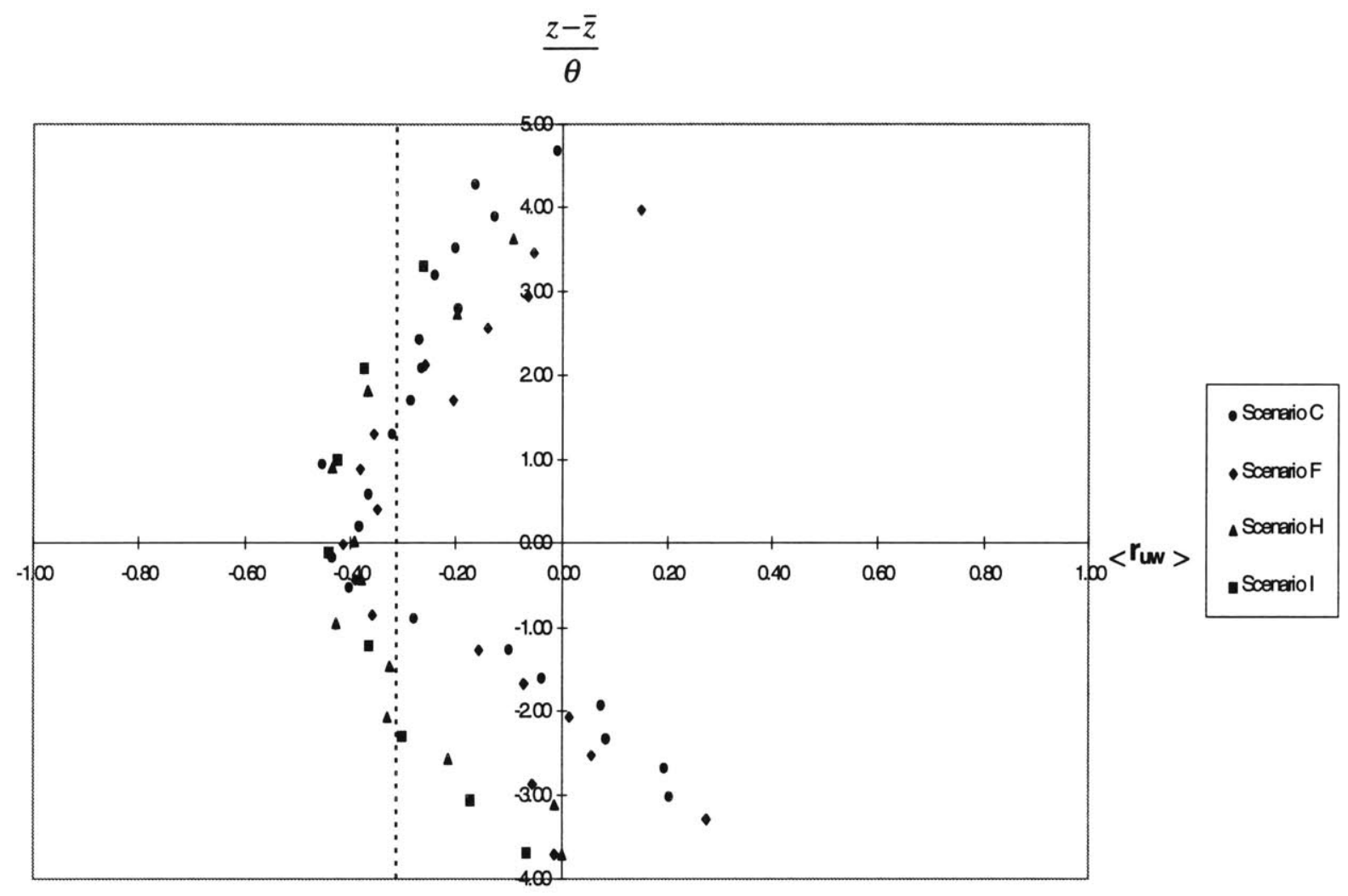

Figure 3.19. Correlation coefficient profiles through the mixing layer for all flow scenarios without the monami. The dashed line indicates the maximum magnitude of $r_{u w}$ observed in boundary layer flow ( -0.3).

Thus, regardless of the degree of plant submergence, the vertical extent of the vortices in a waving canopy is approximately $6 \theta$. This corresponds approximately to the mixing layer thickness (Table 3.1), meaning that above a canopy exhibiting the monami, the structures encompass (vertically) almost all of the mixing layer. In a non-waving canopy, however, the inability of the vortex to penetrate into the canopy leads to a diminished vortex height of between $3 \theta$ and $5 \theta$.

Figures 3.18 and 3.19 also confirm that the vortices are sitting slightly above the geometric center of the mixing layer; the center of the region of $\left|r_{u w}\right|>0.32$ lies approximately 
$0.4 \theta$ above $\bar{z}$. However, the resulting mean velocity increase due to this displacement is only $\sim 0.06 \Delta U$ (Figure 3.6). Since the average $\Delta U / \bar{U}$ ratio is $1.4 \pm 0.3$ (Table 3.1) this mean velocity increase is not nearly large enough to explain vortex velocities of the magnitude observed $(1.1-1.5 \bar{U})$.

\subsubsection{Reverse shear in non-waving canopies}

The observations of an increase in velocity as the bed is approached within non-waving canopies (Figure 3.5) is intriguing. Figure 3.20, a magnified look at the mean in-canopy velocities, highlights the presence of this reverse shear within canopies that do not exhibit the monami. The mean velocity profiles have been shifted by $U_{\min }$, the lowest observed velocity in the canopy (excluding the point nearest the bed) and normalized by $\bar{U}$.

Given the significance of fluid motions immediately above the sediment-water interface in governing chemical, biological and depositional/erosional processes, the near-bed maximum velocity is an important physical parameter. The presence of reverse shear seemingly arises due to the inability of the Reynolds stress of the vortices to penetrate deeply into a non-waving canopy (Figure 3.17). Thus, ignoring small spatial accelerations, the resulting conservation of streamwise momentum in the lower parts of the canopy $\left(z<h_{d}-h_{p}\right)$ reduces to a balance between the exerted drag force and the driving pressure force, supplied by the setup of a surface slope. As the model eelgrass blades (and indeed, actual eelgrass blades) emerge from a bundled stem region, the frontal area of the plants increases with height above the bed; consequently, so too does the exerted drag force. As a result, this region is characterized by a mean velocity that decreases with height above the bed. 


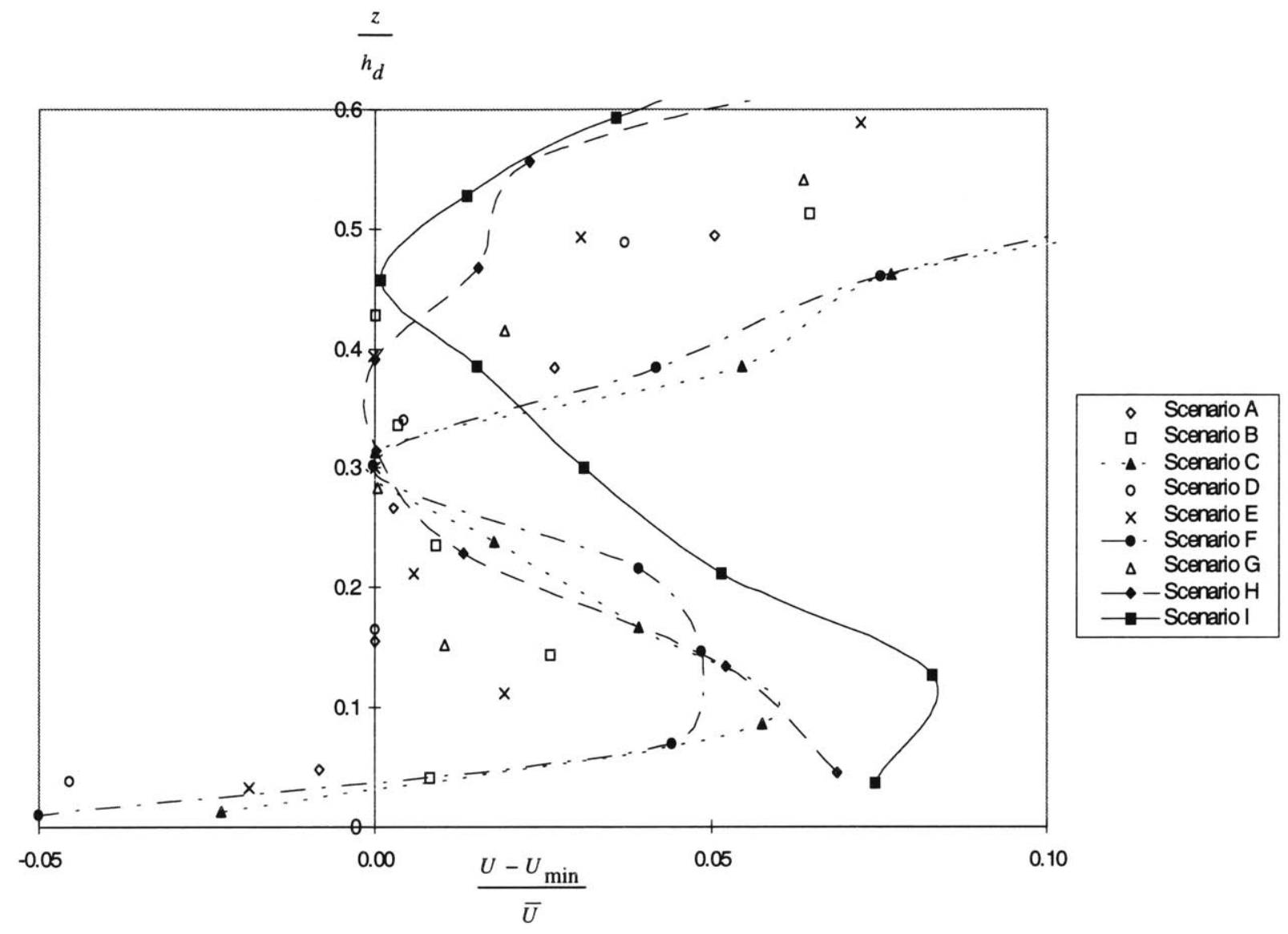

Figure 3.20. Evidence of reverse shear in the non-waving canopies of this study; the mean velocity profiles have been shifted by the minimum velocity and normalized by the mean mixing layer velocity. Vertical velocity profiles of non-waving canopies are highlighted with lines.

Careful examination of the results of Vivoni (1998) shows a similar pattern; regions of reverse shear (of a similar strength to those shown in Figure 3.20) limited to the cases where the monami is nonexistent. However, high positive values of $r_{u w}$ were not observed in his canopy. 


\section{Chapter 4. Further Discussion}

\subsection{Differences between vegetated flow and laboratory mixing layers}

There are several differences between this example of mixing layer flow with that commonly generated in the laboratory - namely, that of two fluid streams initially separated by a splitter-plate. The boundary layers that develop on either side of the splitter plate cause the mixing layer to have a non-zero initial momentum thickness $\left(\theta_{i}\right)$. Downstream distances are generally normalized by $\theta_{i}$ and/or the resulting wavelength of the initial wave instability (which scales upon $\theta_{i}$, according to Equation 1.2). For example, Winant and Browand (1974) detail an initial zone of vortex amalgamation commencing at a distance (downstream of the splitterplate) of approximately 3 wavelengths of the initial wave instability. In the case of the mixing layers generated by aquatic vegetation, the profile has no initial momentum thickness and comparison to downstream distances in pure mixing layer experiments is difficult. Additionally, $\Delta U$ is a function of $x$, meaning the most fundamental mixing layer parameter $(R)$ is not constant.

Preliminary investigations revealed clearly discernible, individual peaks in the frequency spectrum of longitudinal velocity at $x=0.7 \mathrm{~m}, 2.0 \mathrm{~m}, 3.5 \mathrm{~m}$ and $5.0 \mathrm{~m}$ along the model canopy, under the same flow conditions as employed in the preliminary test (Section 3.2). The distinct peaks indicate that vortex merging is not taking place for $x>0.7 \mathrm{~m}$, as the discrete nature of the merging would preclude the presence of a single frequency of velocity oscillations in the domain where merging is occurring. Even if merging was completed over a very small longitudinal distance, an almost instantaneous doubling of mixing layer momentum thickness should be observed (Ho and Huerre, 1984); Figure 3.2 provides evidence that this is not occurring. Seemingly, therefore, the flow field through submerged aquatic vegetation can be thought of as a vortex street with increasing momentum thickness due to turbulent 
entrainment by the vortical structures, but without vortex amalgamation. .However, flow visualization is required as confirmation of the lack of vortex interactions in the generated mixing layer. Ikeda and Kanazawa (1996) made no mention of vortex merging in their flow visualization experiments above a flexible submerged model canopy.

The lack of vortex amalgamation complies with the theoretical criterion of $\Lambda / \delta_{w}<3.5$ for amalgamation to occur by tearing (i.e. the merging mechanism after pairing is complete) in an array of transverse vortices (Raupach et al., 1996), where $\Lambda$ is the vortex spacing and $\delta_{w}$ is the vorticity thickness. The vorticity thickness is approximately equal to 4.5 times the momentum thickness in vegetated flows (Raupach et al., 1996). An estimate of the vortex spacing is obtained from:

$$
\Lambda=\frac{U_{v}}{f}=31.2 \theta\left(\frac{U_{v}}{\bar{U}}\right)
$$

using Equation (1.2). Therefore,

$$
\frac{\Lambda}{\delta_{w}} \approx 7\left(\frac{U_{v}}{\bar{U}}\right)
$$

Since $U_{v} / \bar{U}>1$, vortex amalgamation by tearing is not expected to be occurring at the point of observation (i.e. $x=6.5 \mathrm{~m}$ ).

As discussed in Section 3.5.3.1, the vertical extent of the vortices $\left(\delta_{v}\right)$ in waving canopies is approximately $6 \theta$. From Equation 4.2, the vortex spacing in these waving canopies is between $8.8-10.3 \delta_{w}$, using the relative vortex velocities listed in Table 3.4. Since $\delta_{w} \approx 4.5 \theta$ and $\delta_{v} \approx 6 \theta$ in waving canopies, $\delta_{w} / \delta_{v} \approx 0.75$. Therefore, the vortex spacing varies between $6.6-7.7 \delta$ in the waving canopies of this study. This corroborates the findings of Ikeda and 
Kanazawa (1996), whose flow visualization shows the vortex spacing to be, at the very least, $5 \delta_{\nu}$ in a waving canopy. With the reduced size of the vortices above non-waving canopies, the vortex spacing is as much as $12.7 \delta_{v}$ (Scenario C).

\subsection{Effects of depth limitation}

As discussed briefly in Chapter 1, an important aspect of this research is distinguishing between vegetated flows in the atmosphere and those in aquatic systems. The imposition of the free surface in aquatic flows represents the most important difference. The presence of the free surface implies that we are frequently no longer dealing with, literally, a free shear layer. Interestingly, even when the free surface clearly prevents further development of the shear layer, the monami frequency is still in agreement with Equation 1.2 for a free shear layer. This indicates that Equation 1.2 reflects purely a kinematic constraint and is independent of the evolution of the mixing layer.

In contrast, in atmospheric canopies there is poor agreement between the observed frequency of vortex generation and linear instability theory (Equation 1.2) and the estimated streamwise separation of the vortices is approximately half that predicted, based on the thickness of the mixing layer. The authors surmise that several high-Reynolds-number processes are responsible for this discrepancy; the emergence of modes (due to nonlinearities) other than the fastest-growing mode as predicted by linear analysis and the additional entrainment in a turbulent mixing layer are possibly responsible. Because wind velocities and terrestrial length scales are much greater than the currents and length scales found in aquatic vegetated flow, it is reasonable to assume that such high-Reynolds-number processes are not occurring in vegetated aquatic flows, and certainly not in the laboratory flume. In addition, mixing layers generated by terrestrial vegetation are superimposed upon an atmospheric boundary layer of much greater scale. There is therefore an interaction between the coherent structures of the mixing layer and the turbulence of the atmospheric boundary layer. However, 
in the majority of vegetated aquatic flows (i.e. $H / h_{d}<\sim 10$ ), this is not the case, and the mixing layer extends through most (if not all) of the water column.

\subsection{Vortex kinematics}

The signature of the monami as a localized region of forward bending of the plants indicates that an increase in longitudinal velocity occurs at the top of the canopy as a vortex passes. This implies that the convective speed of the vortices minus the longitudinal velocity associated with vortex circulation is larger than the mean velocity at the top of the canopy. As an example, consider the vertical velocity records at $z_{w r m s}$ for Scenario A. The $w_{r m s}$ at this height, approximately $1.2 \mathrm{cms}^{-1}$, can be taken as an estimate of the circulation speed within the vortex. In this flow scenario, the vortices are propagating at approximately $10.3 \mathrm{cms}^{-1}$ (Table 3.4 ), and $U_{h} \sim 4.5 \mathrm{cms}^{-1}$ (Figure 3.5). As the vortex passes, the streamwise velocity recorded at $z=h$ increases to $(10.3-1.2)=9.1 \mathrm{cms}^{-1}$, significantly greater than $U_{h}$. Although vortex circulation does therefore not dominate streamwise velocity fluctuations, it is a significant cause of vertical velocity fluctuations and is the mechanism behind the strong vertical momentum fluxes in the mixing layer.

The rapid convective velocity of the vortices remains counterintuitive. Although the canopy drag is continually present, it can still not explain the disproportionate effect of the high-stream velocities on the vortex speed. The vortices are located, more or less, in the vertical center of an approximately symmetrical mixing layer. This means that integration of the mean velocity profile over the vertical extent of the coherent structure (as determined by Figure 3.16) gives an average velocity to which the vortex is exposed of almost exactly $\bar{U}$. The comparatively high velocity of the vortices $\left(U_{v} / \bar{U}\right.$ up to 1.5$)$ is inconsistent with laboratorygenerated mixing layers without the vegetation drag, although a similar phenomenon is seen in terrestrial canopies. 
The observation of several 'monami channels' in such a narrow flume is certainly surprising. This lack of lateral uniformity of the monami is, however, consistent with waving phenomena in terrestrial canopies (e.g. waving wheat fields) and the three-dimensionality of the coherent structures observed by Ikeda and Kanazawa (1994) above a flexible model canopy. Conversely, in splitter-plate-generated mixing layers the coherent structures have a quasi-twodimensional structure (Browand and Troutt, 1990).

\subsection{Frequencies of plant vibration}

Due to the low currents through aquatic vegetation, the monami frequency is generally considerably lower than the natural frequency of vibration of the vegetation $(0.18 \mathrm{~Hz})$. Finnigan and Mulhearn (1978) showed that terrestrial vegetation absorbs wind momentum preferentially at the natural frequency of vibration of the plant structure. Thus, while the plant waving in this study may have a minor component at the natural frequency, the instability frequency of the mixing layer dominates plant motion. It is suspected that the low-amplitude lateral oscillation of the model plants of Vivoni (1998) corresponded to their natural frequency. Spectra of both longitudinal and vertical velocity in this study (examples of which are shown in Figures 3.8 and 3.10 respectively) show no evidence of oscillations at a frequency of $0.18 \mathrm{~Hz}$. This is expected given the forced nature of plant waving that is suspected.

\subsubsection{Effect of plant waving}

As demonstrated by Figures 3.16 and 3.17 , there is minimal Reynolds stress penetration into the non-waving canopies of this study ( $h_{p \text {,waving }} \sim 1.9 h_{p, n o n-w a v i n g}$ ). This indicates that the monami cannot be thought of as a completely passive response to the flow. Although Vivoni (1998) found no significant difference in the turbulence structure based on the presence of the monami, his vegetation was very rigid and exhibited very low-amplitude waving, in contrast to this study. 


\subsection{Length scale prediction}

The clear mixing layer nature of vegetated flows means that there are two sets of length scales: those intrinsic to the system (e.g. plant height, water depth) and those that are functions of the mixing layer flow (e.g. momentum thickness, mixing layer thickness). With measurement of the latter being far from a trivial prospect, it is desirable to determine processbased empirical relationships to obtain some predictive capability.

It is expected that the momentum thickness of the mixing layer will depend upon the depth of the surface layer above the canopy (i.e. $H-h_{d}$ ), as this is the region in which the mixing layer is free to grow before the free surface can restrict further growth. Linear regression of the observed values of $\theta$ with $H-h_{d}$, both non-dimensionalized by $h_{d}$, shows an excellent correlation, as demonstrated in Figure 4.1. When the regression line is forced through the origin, since emergent vegetation $\left(H-h_{d}=0\right)$ does not create a mixing layer in the flow, $\theta \approx 0.16\left(H-h_{d}\right)$. 


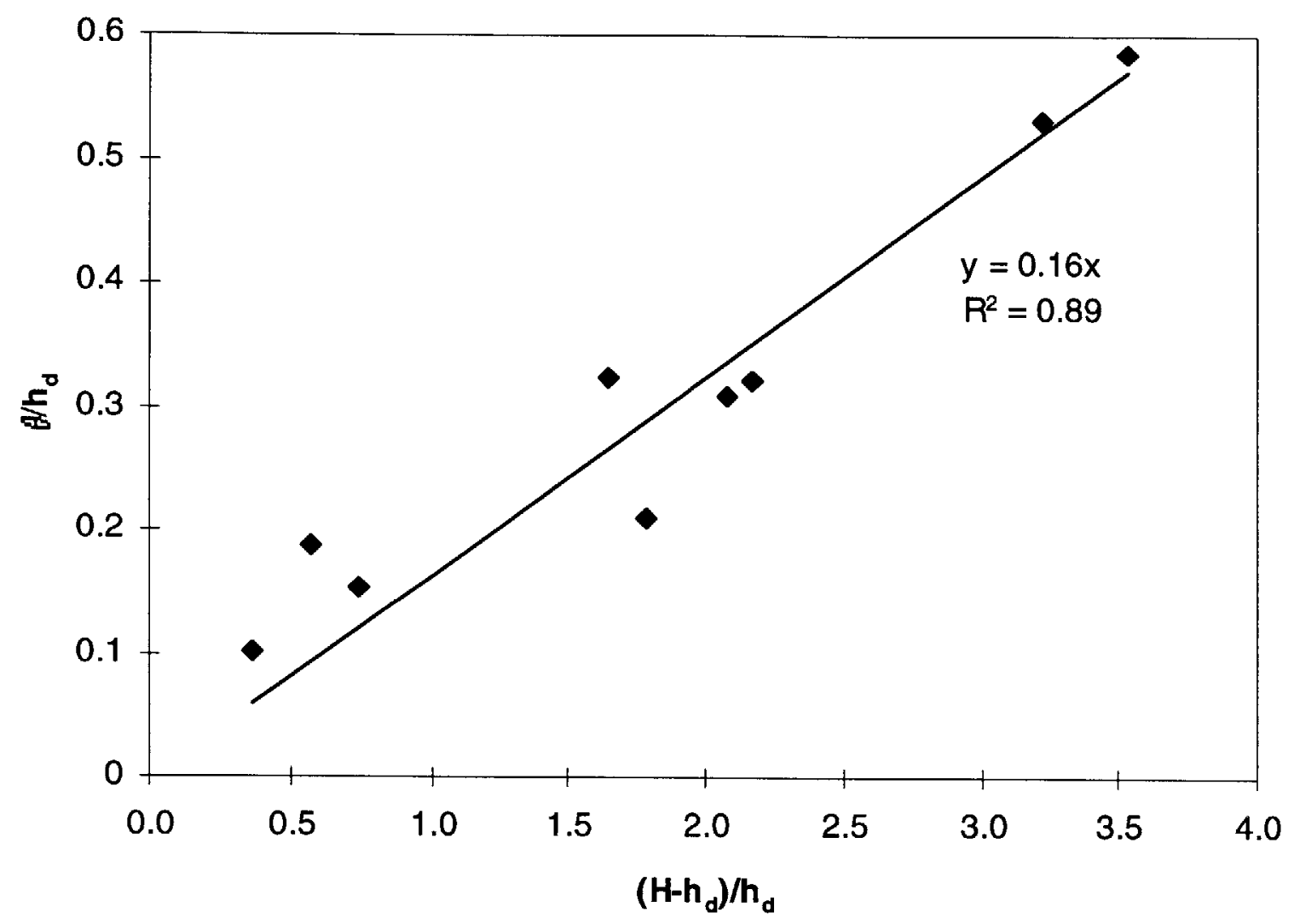

Figure 4.1. Evidence of the linear relationship between the observed momentum thicknesses in this study and the depth of the surface layer above the canopy.

The ability to estimate the mixing layer momentum thickness from $h_{d}$ and $H$ (two easily obtainable parameters) using this relationship simplifies things enormously. Furthermore, two velocity measurements (to obtain $U_{I}$ and $U_{2}$ ) allow the estimation of the mean velocity profile, based on the collapse in Figure 3.6.

\subsection{Future work}

The results of this study have raised a number of questions that deserve attention. Firstly, the propagation of the generated vortices is of particular interest, as their high velocity 
is as yet unexplained. A flow visualization study would complement the study of Ikeda and Kanazawa (1996) and go a long way in determining the nature of the streamwise mass transport associated with the vortices.

Secondly, the scalar fluxes that exist in and above the canopy must be quantified, with particular attention being paid to comparison with pure mixing layers. In such a study, it is critical that the generated shear layer be made turbulent $\left(R e_{h m}>17000\right.$, in line with the estimates of Koochesfahani and Dimotakis (1986)). As the transition from a laminar to a turbulent mixing layer greatly increases the amount of small scale mixing, it is vital that we have conditions representative of the field. For example, a rough field estimate of $R e_{h m}$ from Grizzle et al. (1996) is $2 \times 10^{5}$, well above the upper limit of the mixing transition; it is expected that in the majority of cases in the field, the mixing layer would indeed be turbulent. This is the Reynolds number criterion that should be satisfied in the scaling down of an experimental model when focusing upon scalar fluxes. It is worth noting, however, that the effect of the mixing transition on momentum transport and shear layer development is far less pronounced than the effect on scalar fluxes (Moser and Rogers, 1991), so the work of this study is certainly not invalidated.

Finally, the growth of mixing layers above real seagrass meadows must be evaluated. Although it is suspected that the sidewalls of the flume are not responsible for the asymptotic growth of the generated mixing layers, this must be confirmed by a field study. In horizontal mixing layers generated along the edges of vegetated areas, the vortices that develop fail to grow above a certain size, despite the lack of boundary influence (Pasche, E., pers. comm., 25 May 2000). The thickness of the mixing layer (and hence the size of the coherent structures within it) is expected to strongly regulate vertical fluxes of momentum and scalar quantities in the water column; consequently, knowledge of the fully-developed mixing layer thickness is critical. 


\section{Conclusion}

Like terrestrial vegetated flows, aquatic flow through submerged vegetation can be patterned nicely on that of a mixing layer. The vertical non-uniformity of the vegetation drag creates an inflectional velocity profile, which compares very favorably to hyperbolic tangent velocity profiles generated in splitter-plate experiments. Analysis of mean velocity and turbulent statistics confirms the validity of a mixing layer analogy for these vegetated flows, previously regarded as perturbed bottom boundary layers.

The inflectional profile created by the vegetation results in the development of a vortex street of Kelvin-Helmholtz instabilities. The instabilities are strongly three-dimensional, as the presence of several 'monami channels' indicates. These vortices are responsible for very efficient vertical transport of streamwise momentum in the mixing layer, as evidenced by the strong correlation between horizontal and vertical turbulent fluctuations. This correlation is invariably greater than that typically found in bottom boundary layers. Thus, these structures dominate the vertical transport of momentum and it can be inferred that they also dominate vertical scalar fluxes in the flow.

The advection speed of a vortex, greater than the mean velocity of the mixing layer, is sufficient to cause an instantaneous velocity increase at the top of the canopy as it passes. Thus, localized regions of forward plant deflection progress smoothly along the canopy. This is indeed the coherent waving phenomenon known as the monami. The downstream advection of this vortex street leads to strong, periodic oscillations in streamwise and vertical velocities, the frequencies of which are invariant over depth and clearly discernible in power spectra. The monami is not present under all flow conditions, restricted to flows where the velocity at the top of the canopy is greater than some threshold value. This threshold velocity depends strongly on flow depth and plant flexibility. 


\section{References}

1. Ackerman, J.D. and A. Okubo (1993) Reduced mixing in a marine macrophyte canopy, Func. Ecol., 7 : 305-309.

2. Browand, F.K. and Troutt, T.R. (1985) The turbulent mixing layer : geometry of large vortices, J. Fluid. Mech., 158 : 489-509.

3. Browand, F.K. and Troutt, T.R. (1990) A note on spanwise structure in the twodimensional mixing layer, J. Fluid. Mech., 97 : 771-781.

4. Brown, G.L. and Roshko, A. (1974) On density effects and large structure in turbulent mixing layers, J. Fluid Mech., 64 : 755-816.

5. Buchhave, P., George, W.K. Jr and Lumley, J.L. (1979) The measurement of turbulence with the laser-doppler anemometer, Ann. Rev. Fluid Mech., 11 : 443-503.

6. Chandler, M., Colarusso, P. and Buchsbaum, R. (1996) A study of eelgrass beds in Boston Harbor and northern Massachusetts bays, Office of Research and Development, U.S. Environmental Protection Agency, Narragansett, RI.

7. Dantec Measurement Technology (1990a) User's guide : 60X series FiberFlow, Skovlunde, Denmark.

8. Dantec Measurement Technology (1990b) Floware : User's guide, Skovlunde, Denmark.

9. den Hartog, C. (1970) Seagrasses of the world, North-Holland, Holland. 
10. DePaoli, L. (1999) Numerical modelling of wetland hydrodynamics Master's Thesis, Department of Civil and Environmental Engineering, Massachusetts Institute of Technology.

11. Dimotakis, P.E. and Brown, G.L. (1976) The mixing layer at high Reynolds number : large structure dynamics and entrainment, J. Fluid Mech., 78 : 535-560.

12. Dimotakis, P.E., Debussy, F.D. and Koochesfahani, M.M. (1981) Particle streak velocity field measurements in a two-dimensional mixing layer, Phys. Fluids, 24(6) : 995-999.

13. Durst, F., Melling, A. and Whitelaw, J.H. (1981) Principles and practice of laser-doppler anemometry, Academic Press Inc., London.

14. Finnigan, J.J. (1979a) Turbulence in waving wheat : I. Mean statistics and honami, Boundary-Layer Meteorology, 16 : 181-211.

15. Finnigan, J.J. (1979b) Turbulence in waving wheat : II. Structure of momentum transfer, Boundary-Layer Meteorology, 16 : 213-236.

16. Finnigan, J.J. and Mulhearn P.J. (1978) Modelling waving crops in a wind tunnel, Boundary-Layer Meteorology, 14 : 253-277.

17. Fonseca, M. (1998) Exploring the basic pattern expression in seagrass landscapes, Ph.D. thesis, University of California, Berkeley.

18. Fonseca, M.S., Fisher, J.S., Zieman, J.C. and Thayer, G.W. (1982) Influence of the seagrass Zostera Marina (L.) on current flow, Estuarine, Coastal and Shelf Science., 15 : 351-364. 
19. Fonseca, M.S. and Kenworthy, W.J. (1987) Effects of current on photosynthesis and distribution of seagrasses, Aq. Bot., 27 : 59-78.

20. Gambi, M.C., Nowell, A.R.M. and Jumars, P.A. (1990) Flume observations on flow dynamics in Zostera marina (eelgrass) beds, Mar. Ecol. Prog. Ser., 61 : 159-169.

21. Gao, W., Shaw, R.H. and Paw U, K.T. (1989) Observation of organized structure in turbulent flow within and above a forest canopy, Boundary-Layer Meteorology, 47 : 349377 .

22. Gerhart, P.M., Gross, R.J. and Hochstein, J.I (1992) Fundamentals of fluid mechanics, Addison-Wesley Publishing Company, USA.

23. Grizzle, R.E., F.T. Short, C.R. Newell, H. Hoven and L. Kindblom (1996) Hydrodynamically induced synchronous waving of seagrasses : 'monami' and its possible effects on larval mussel settlement, Institute for Agricultural and Biology. J. Exp. Mar. Biol. Ecol., 206 : 165-177.

24. Ho, C-M and Huerre, P. (1984) Perturbed free shear layers, Ann. Rev. Fluid Mech., 16, 365-424.

25. Ho, C-M, Zohar, Y., Foss, J.K. and Buell, J.C. (1991) Phase decorrelation of coherent structures in a free shear layer, J. Fluid Mech., $230: 319-337$.

26. Holmes, P., Lumley, J.L. and Berkooz, G. (1996) Turbulence, coherent structures, dynamical systems and symmetry, Cambridge University Press, Cambridge, UK. 
27. Ikeda, S. and Kanazawa, M. (1996) Three-dimensional organized vortices above flexible water plants, J. Hydraul. Eng., 122(11) : 634-640.

28. Inoue, E. (1995) Studies of phenomena of waving plants ('honami') caused by wind. Part 1. Mechanism and characteristics of waving plants phenomena, J. Agric. Meteorol. (Tokyo), $11:$ 18-22 (in Japanese).

29. Koochesfahani, M.M and Dimotakis, P.E. (1986) Mixing and chemical reactions in a turbulent liquid mixing layer, J. Fluid Mech., 170 : 499.

30. Kouwen, N. and Li, R-M. (1980) Biomechanics of vegetative channel linings, J. Hyd. Div., ASCE, 106(HY6) : 1085-1103.

31. Kouwen, N. and Unny, T.E. (1973) Flexible roughness in open channels, J. Hyd. Div., ASCE, 99(HY5) : 713-727.

32. Kundu, P.K. (1990) Fluid mechanics. Academic Press, San Diego, California.

33. Lopez, F. and Garcia, M. (1997) Open-channel flow through simulated vegetation : turbulence modelling and sediment transport, Wetlands Research Program Technical Report WRP-CP-10, US Army Corps of Engineers, Washington, DC.

34. Moser, R.D. and Rogers, M.M. (1991) Mixing transition and the cascade to small scales in a plane mixing layer, Phys. Fluids A., 3(5) : 1128-1134.

35. Murota, A., T. Fukuhara and Sato, M. (1984) Turbulence structure in vegetated open channel flows, Journal of Hydroscience and Hydraulic Engineering, 2(1) : 47-61. 
36. Nepf, H.M. (1999) Drag, turbulence and diffusion in flow through emergent vegetation, Water Resources Research, 35(2), 479-489.

37. Niklas, K.J. (1992) Plant biomechanics, Univ. Chicago Press, Chicago.

38. Panides, E. and Chevray, R. (1990) Vortex dynamics in a plane moderate-Reynoldsnumber shear layer, J. Fluid Mech., 214 : 411-435.

39. Pantin, H.M., Hamilton, D. and Evans, C.D.R. (1981) Secondary flow caused by differential roughness, Langmuir circulations, and their effect on the development of sand ribbons, Geo-Marine Letters, 1 : 255-260.

40. Raupach, M.R., Finnigan. J.J. and Brunet, Y. (1996) Coherent eddies and turbulence in vegetation canopies : the mixing layer analogy, Boundary-Layer Meteorology, 78 : 351382.

41. Raupach, M.R. and Shaw R.H. (1982) Averaging procedures for flow within vegetation canopies, Boundary-Layer Meteorology, 22 : 79-90.

42. Rogers, M.M. and Moser, R.D. (1994) Direct simulation of a self-similar turbulent mixing layer, Phys. Fluids A, 6 : 903-923.

43. SonTek (1995) Frequently asked ADV questions, SonTek technical notes, San Diego, CA.

44. SonTek (1996) ADV : Acoustic Doppler Velocimeter, SonTek technical notes, San Diego, CA. 
45. SonTek (date unknown) SonTek Application Notes - ADV for high-resolution applications, San Diego, CA.

46. Thorpe, S.A. (1971) Experiments on the instability of stratified shear flows : Miscible fluids, J. Fluid. Mech., 46 : 299-319.

47. Vivoni, E.R. (1998) Turbulence structure of a model seagrass meadow, Masters Thesis, Department of Civil and Environmental Engineering, Massachusetts Institute of Technology.

48. Vivoni, E.R., Nepf, H.M. and Koch, E.W. (1997) Chincoteague eelgrass report : preliminary data analysis, unpublished document.

49. Voulgaris, G and Trowbridge, J.H (1997) Evaluation of the Acoustic Doppler Velocimeter (ADV) for turbulence measurements, J. Atm. Ocean. Technol., 15, 272-289.

50. Wallace, S., Luketina, D. and Cox, R. (1998) Large scale turbulence in seagrass canopies, Proc. $13^{\text {th }}$ Australasian Fluid Mechanics Conference, Monash University, Melbourne, Australia, 973-976.

51. Winant, C.D. and Browand, F.K. (1974) Vortex pairing : the mechanism of turbulent mixing layer growth at moderate Reynolds number, J. Fluid Mech., 63(2) : 237-255

52. Zavistoski, R. (1994) Hydrodynamic effects of surface piercing plants, Masters Thesis, Department of Civil and Environmental Engineering, Massachusetts Institute of Technology. 
APPENDIX A1 : MATLAB code for calculation of mean and turbulent flow parameters from ADV data files

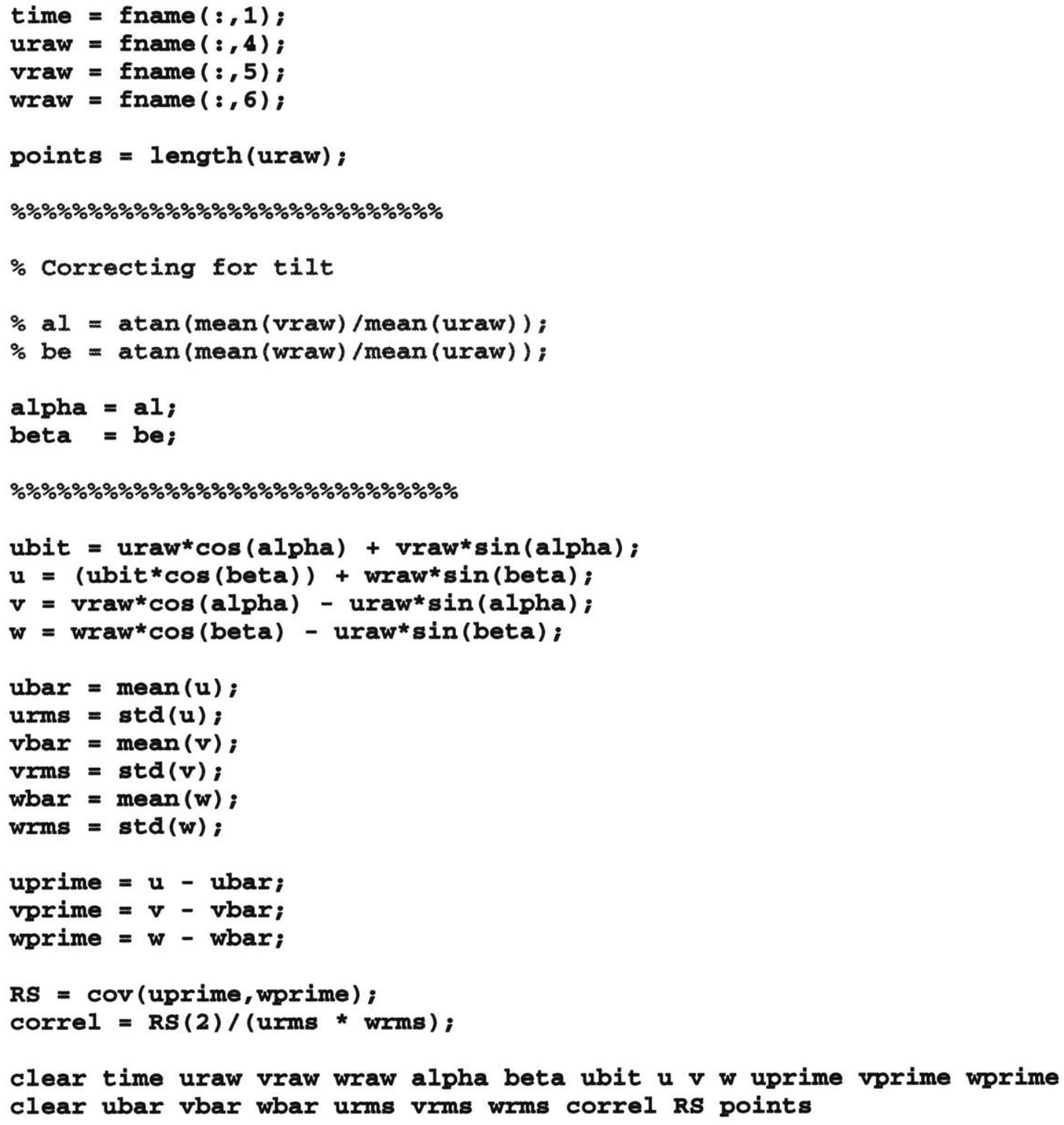


APPENDIX A2 : MATLAB code for calculation of mean and turbulent flow parameters from LDV data files

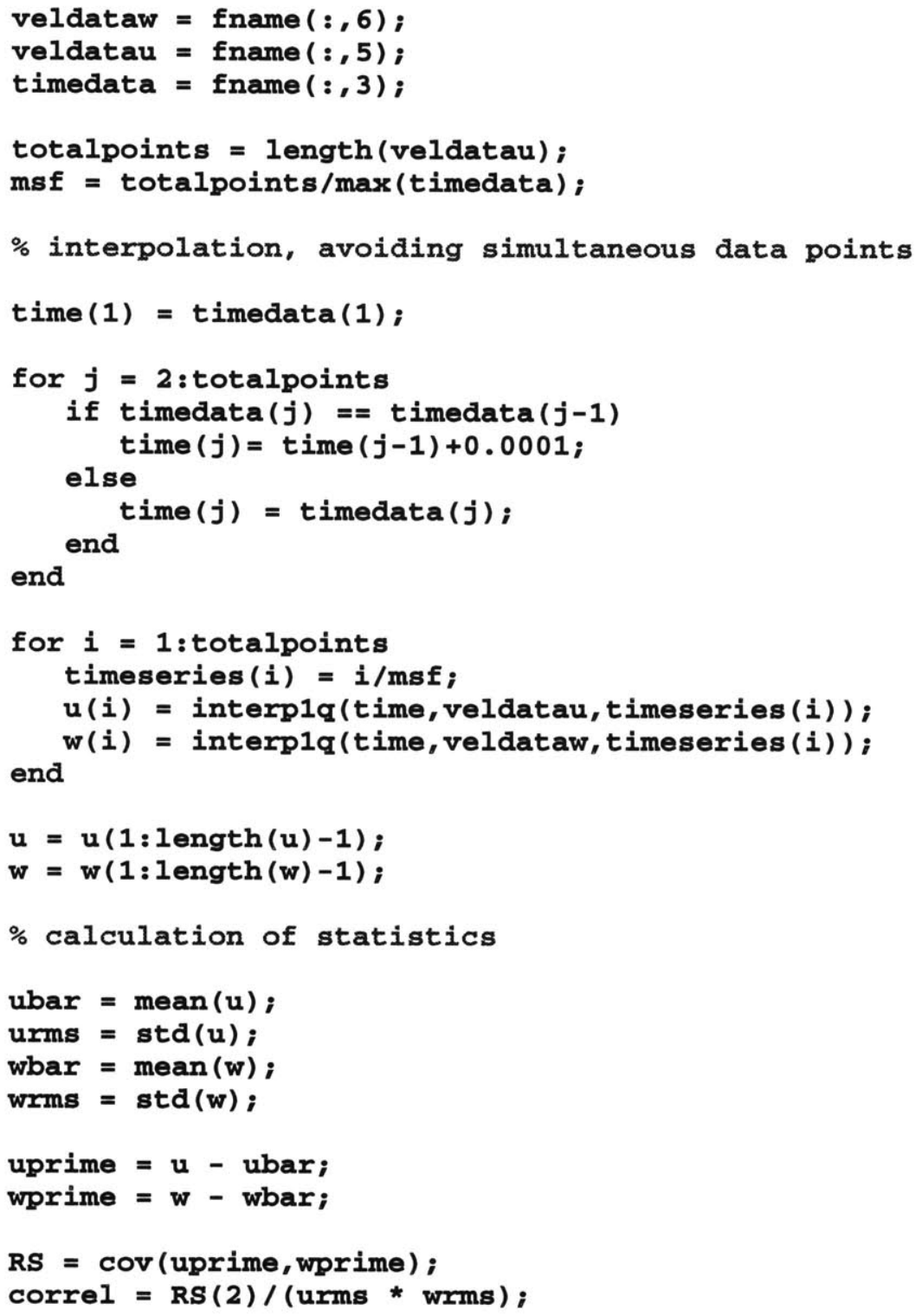


APPENDIX B: MATLAB code for calculation of smoothed spectra

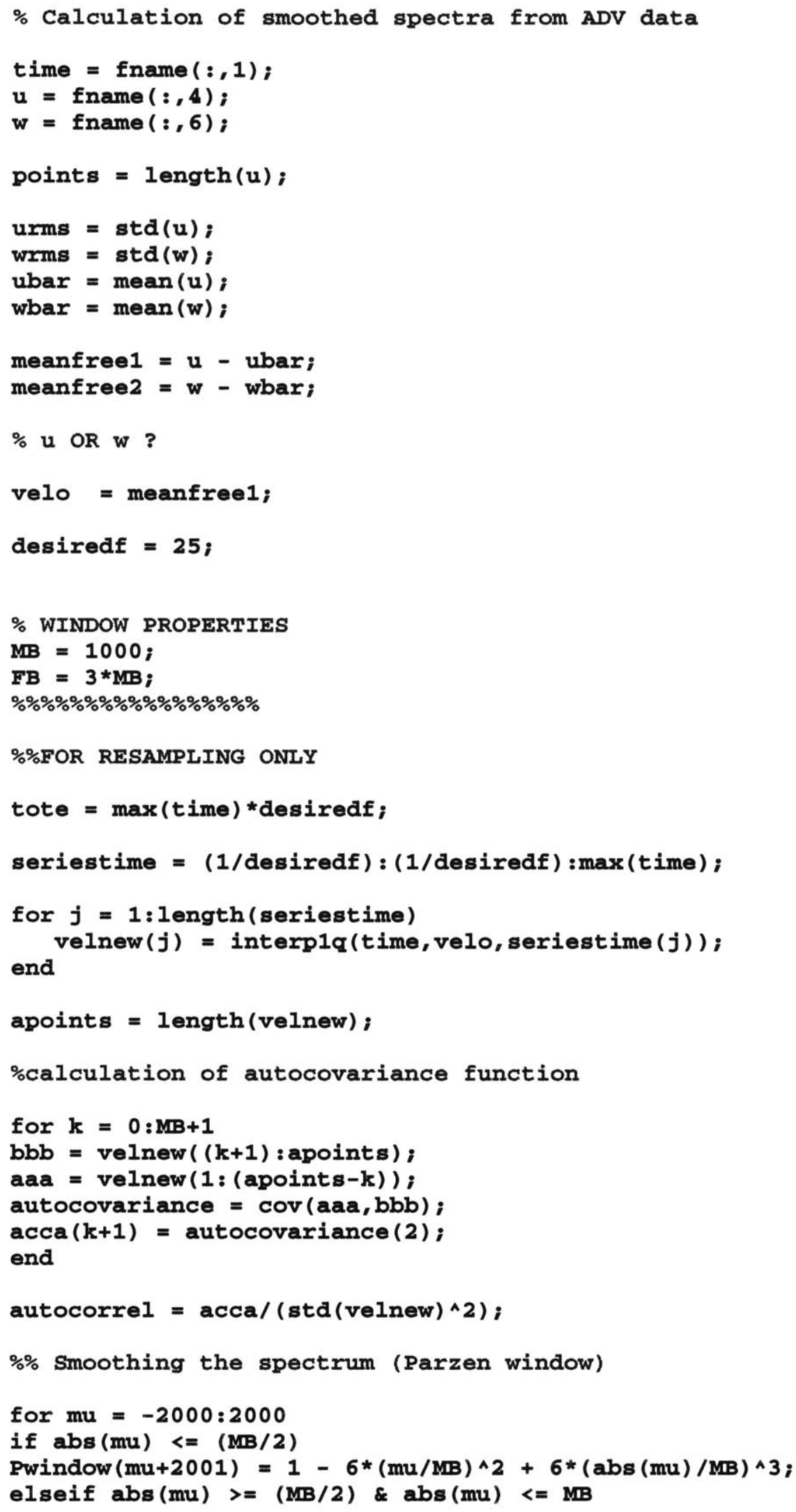




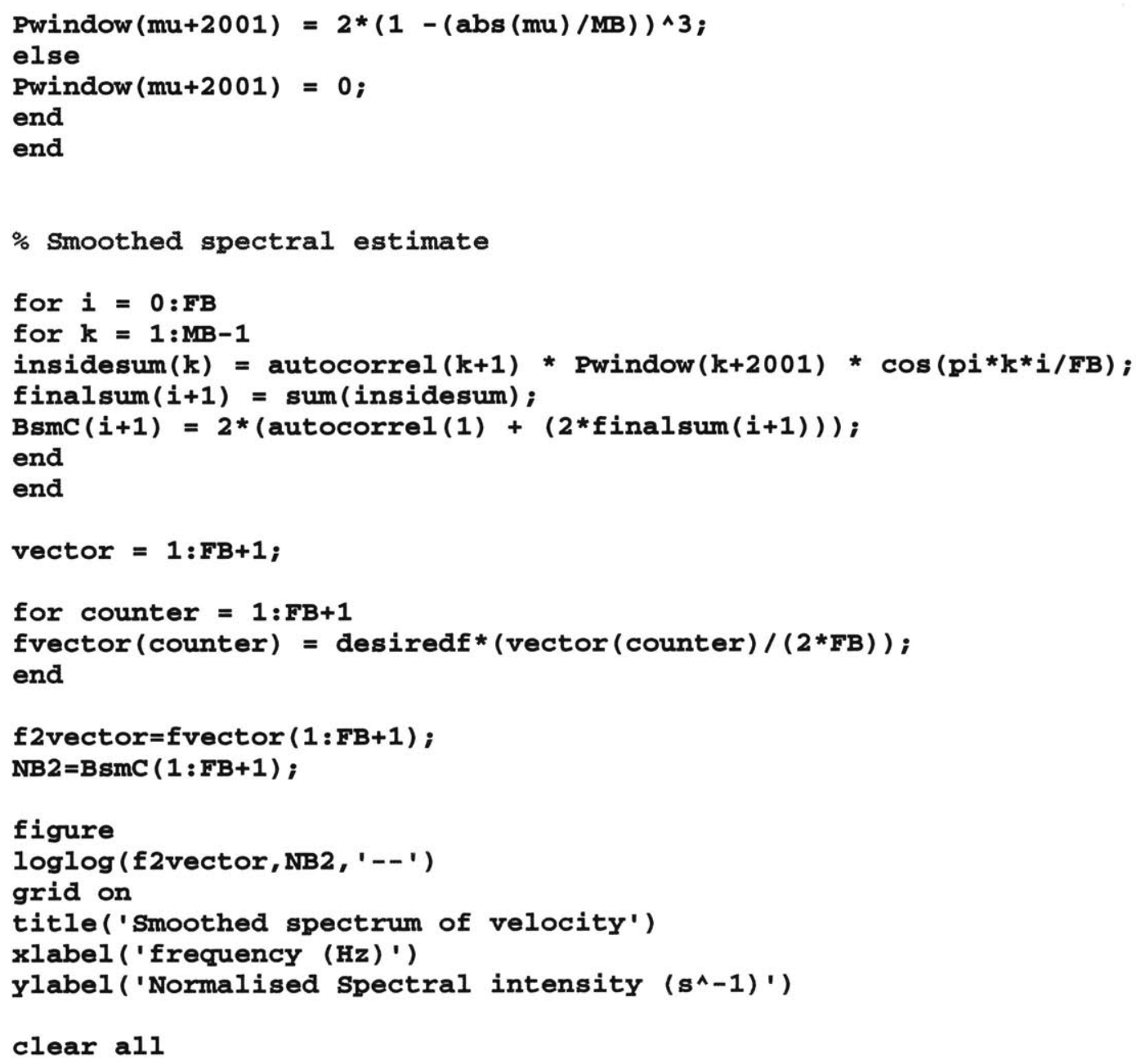


APPENDIX C: MATLAB code for estimation of vortex velocity

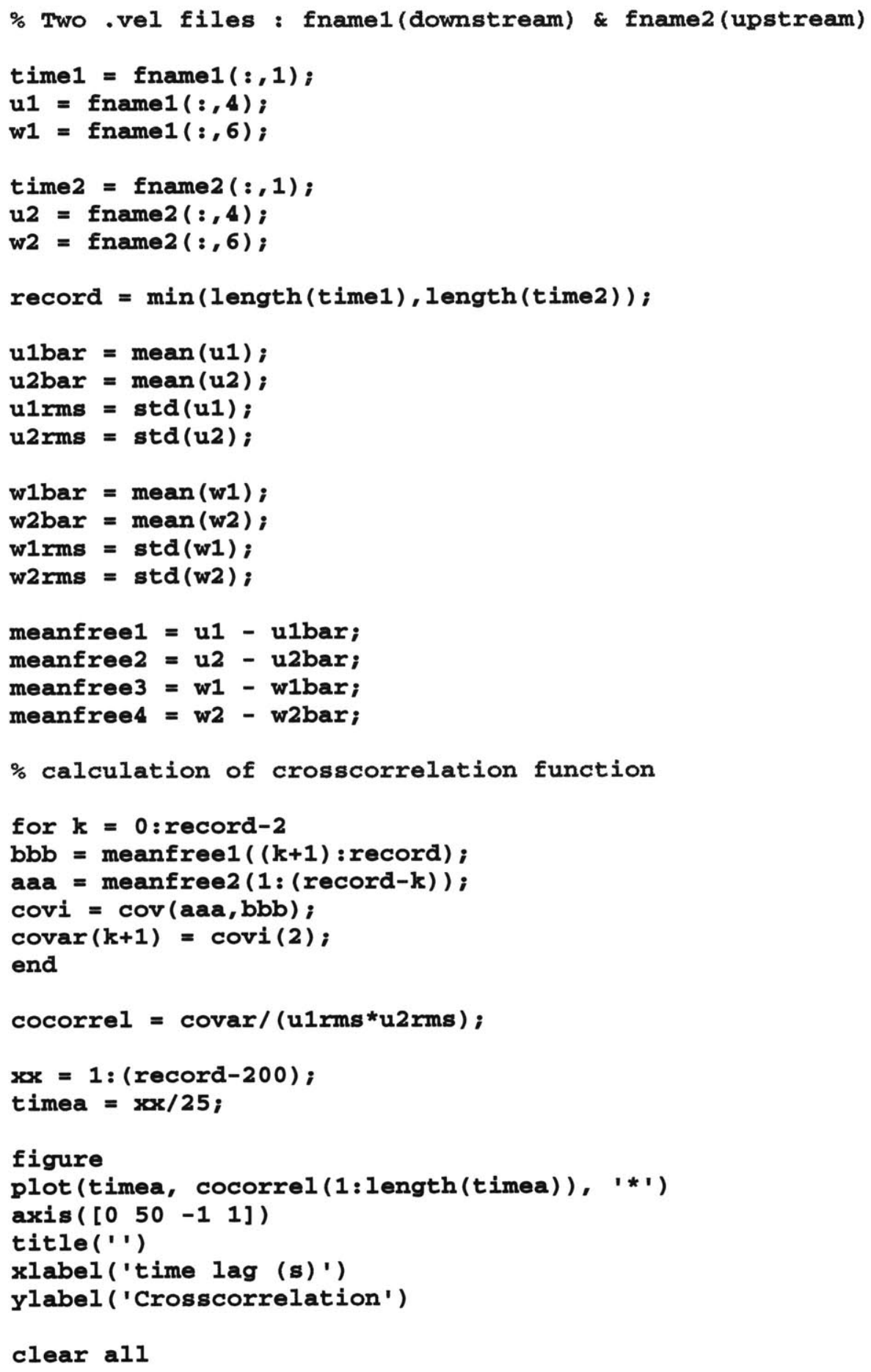

